\title{
Low Latency Compressive Sensing using Multi-resolution Analysis In Radar Signal Processing
}

\author{
by \\ Assem M. Assem, M.Sc. \\ A dissertation submitted to the \\ Faculty of Graduate and Postdoctoral Affairs \\ in partial fulfillment of the requirements for the degree of
}

Doctor of Philosophy in Electrical Engineering

Ottawa-Carleton Institute for Electrical and Computer Engineering

Department of Systems and Computer Engineering

Carleton University

Ottawa, Ontario

August, 2017

(C) Copyright

Assem M. Assem, 2017 


\section{Abstract}

The digital world becomes dominated by electronic devices. The demand for higher speeds is growing every day which implies a higher demand for the signal acquisition and processing speeds and a wider signal bandwidth. The analog-to-digital converter (ADC) is the first step in the digital world. As stated by the Shannon/Nyquist sampling theorem, the ADC must sample the signal at a rate two times faster than the signal bandwidth in order to avoid data loss. However, the advances of ADC cannot always meet these demands. Therefore, considerable research has been done to find new approaches to acquiring the signal at a rate lower than the Nyquist rate (sub-Nyquist) while capturing the data with no losses or distortion. In recent years, compressive sensing (CS) has come to light as a new signal processing paradigm. CS exploits signal sparsity characteristics to acquire the signal using a number of samples much lower than the Nyquist rate. Our focus is studying CS and its applications to radar. We study different successful CS practical implementation approaches. However, these approaches lead to an undesired high-latency in the computation and signal reconstruction.

This thesis has four main contributions that build on each other. First, we study the effect of prior information on the CS recovery performance and introduce a new formula to improve the utilization of the prior information in CS pulse radar. Second, we exploit prior information and multi-resolution analysis properties to introduce a low-latency sub-Nyquist adaptive sampling algorithm. The proposed algorithm is a modified version of CS sampling approach called Xampling but uses Haar wavelets. The Haar wavelet based approach is found to be useful to extract target range information for radar applications that requires only target range detection. Third, we modified our Haar approach by using S-transform bases instead of Haar bases. The S-transform based algorithm is capable of analyzing signal frequency and phase that gives the algorithm the ability to extract target range and speed information in 
a single reconstruction step. The S-transform approach provides a reasonable balance between the number of samples and the reconstruction processing time. Fourth, we study the recovery guarantees for the S-transform algorithm. We introduce a mathematical proof to derive an upper bound for the number of detectable targets. 


\section{Acknowledgments}

The Ph.D. was not only my dream but also my father's and my mother's dream. As I'm writing these words now, truly I cannot believe that this dream has come true. After finishing my master degree, my goal was to apply for Ph.D. studies in my home country, Egypt. When I was doing my paper work for the Ph.D. application, I met my friend, Ahmad Foad, who told me that there are some changes in the postgraduate studies scholarship rules that allow me to apply for studying my Ph.D. abroad. That moment was a turning point in my life. It was another dream to travel abroad.

In my Ph.D. trip in Canada, I have met different people and different cultures that enriched me. Many people have contributed to my Ph.D. success, all in different ways. I would like to take this opportunity to thank them all for helping me make this dream come true.

First and foremost, thanks ALLAH, the most gracious the most merciful for all his blessings that I cannot count. I would like to thank ALLAH for three blessings.

The first blessing is my parents whom I do not believe that any language in the world has words yet to express my gratitude to. My father kept encouraging me to apply for graduate studies since I finished my undergraduate studies. To be honest, without him, I could never have done my Ph.D. Also my mother, whom I wish she has been here with me now. She was like a candle lighting up my way. May ALLAH bless her and dwell her the best place in the paradise.

The second blessing is my wife. Even in my best dreams, I could never imagine meeting a person like her to share my life with. She endured a lot of stress and hardships with me with smile and love. Whenever I need support she is there, with her charming smile, to give me all the positive power that I need to go forward.

The third blessing is my supervisor, Prof. Richard Dansereau. I have learned from him about scientific ethics and morals. He always has good words for me and motivates me to pursue my research, especially when I was very discouraged. I will take your teachings with me throughout my scientific career. 
For anyone whom ALLAH gives these three blessings, a Ph.D. is a logical result. I also want to apologize to my little angels, Lamar, Layan and Lara for my absence and for the stressful moments that they had because of my Ph.D. promising to compensate them with great hugs and a lot of fun.

A very special gratitude goes out to my friend, Saad EL-Gayar, Who helped me a lot to finish the mathematical proofs in my thesis. 


\section{Table of Contents}

Abstract $\quad$ ii

Acknowledgments $\quad$ iv

Table of Contents vi vi

List of Figures $\quad$ ix

List of Tables $\quad$ xi

Nomenclature $\quad$ xiii

1 Introduction $\quad 1$

1.1 Efficient Sampling . . . . . . . . . . . . . . . . 1

1.2 Problem Statement and Research Objective . . . . . . . . . . . . . 2

1.2.1 Hypothesis Statement . . . . . . . . . . . . 3

1.3 Contributions ......................... 4

1.3.1 CS Radar using Prior Information . . . . . . . . . . . . . . . 4

1.3.2 Low-Latency Sub-Nyquist Sampling Algorithm . . . . . . . . . 4

1.3.3 Frequency and Phase Analysis using S-transform . . . . . . . 5

1.3.4 Recovery Guarantees for the S-transform matrix . . . . . . . 5

1.4 Publications for this Dissertation . . . . . . . . . . . . 5

1.5 Dissertation Organization . . . . . . . . . . . . 6

2 Radar and Compressive Sensing Concepts $\quad 8$

2.1 Radar In Brief . . . . . . . . . . . . . . . . . . . . . . 8

2.1.1 Radar Structure . . . . . . . . . . . . . . . . . 10

2.1.2 Radar Bandwidth . . . . . . . . . . . . . . . 14 
2.2 Compressive Sensing Background . . . . . . . . . . . . . . . . 14

2.3 CS Radar Detection Performance . . . . . . . . . . . . . . . . . . 19

2.4 Radar Detectors using Compressive Sensing . . . . . . . . . 20

2.5 Sampling Process in CS . . . . . . . . . . . . . . . . . 21

2.6 Conclusion . . . . . . . . . . . . . . . . . . . . . . . . . . 25

3 Compressive Sensing in Pulse Radar using Prior Information $\quad 26$

3.1 Prior Information and Compressive Sensing . . . . . . . . . 26

3.2 Compressive Sensing in Radar Applications Using Prior Information . 28

3.2 .1 Motivations . . . . . . . . . . . . . . . . . . 28

3.2 .2 Proposed Formula. . . . . . . . . . . . . . . . . . 30

3.3 Analysis and Results . . . . . . . . . . . . . . . . . . 31

3.4 Conclusion . . . . . . . . . . . . . . . . . . . 37

4 Adaptive Sub-Nyquist Sampling and Compressive Detection for Pulsed Radar $\quad 38$

4.1 Adaptive Sub-Nyquist Sampling . . . . . . . . . . . . . . 38

4.1.1 Wavelets and Signal Model . . . . . . . . . . . . . . . . 39

4.1.2 Algorithm Motivations . . . . . . . . . . . . . . . . 41

4.1 .3 Algorithm Procedures . . . . . . . . . . . . . . . . 42

4.2 Analysis and Results . . . . . . . . . . . . . . . . 46

4.3 Conclusion . . . . . . . . . . . . . . . . . . 48

5 Frequency and Phase Analysis Using S-transform 51

5.1 Motivations . . . . . . . . . . . . . . . . . . 51

$5.2 \quad$ S-transform Background . . . . . . . . . . . . . . . 52

5.3 Adaptive Sub-Nyquist S-transform Algorithm . . . . . . . . . 55

5.3 .1 Signal Model . . . . . . . . . . . . . . . . . 55

5.3 .2 Target Range Detection . . . . . . . . . . . . . . . 57

5.3.3 Doppler Information and CS Moving Target Indicator (CS-MTI) 59

5.4 Analysis and Results . . . . . . . . . . . . . . . . 60

5.4 .1 Simulation Parameters . . . . . . . . . . . . . . . 60

5.4 .2 Results. . . . . . . . . . . . . . . . . . . . 61

5.4 .3 Discussion . . . . . . . . . . . . . . . . 62

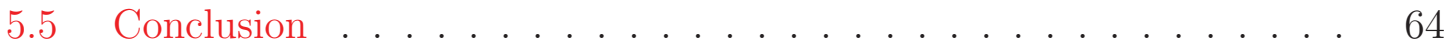


6 Recovery Guarantees for S-transform Matrix 65

6.1 Mathematical background on compressive sensing . . . . . . . . . 65

6.2 Signal model . . . . . . . . . . . . . . . . . . . . . . . . . . . 69

6.3 Main Result . . . . . . . . . . . . . . . . . . 70

6.4 Proof of Theorem $4 \ldots \ldots \ldots 71$

6.4.1 Useful Lemmas . . . . . . . . . . . . . . . . . . . . 71

6.4.2 Theorem 4 proof .................. 73

6.5 Numerical Experiments . . . . . . . . . . . . . . . . . . . 84

6.6 Conclusion . . . . . . . . . . . . . . . . . . 85

7 Conclusion and Future Work $\quad 87$

7.1 Conclusion . . . . . . . . . . . . . . . . . . 87

7.2 Limitations . . . . . . . . . . . . . . . . . . . . . . . 88

7.3 Future Work . . . . . . . . . . . . . . . . . . . . 9 90

$\begin{array}{ll}\text { List of References } & 92\end{array}$ 


\section{List of Figures}

2.1 Example of air surveillance radar. . . . . . . . . . . . . . . . 9

2.2 Block diagram of the basic radar subsystems [1] . . . . . . . . . . 9

2.3 The central operation of the CFAR processor . . . . . . . . . . . . 11

2.4 (a) Typical radar return PSD when clutter and target are present. (b) MTI filter frequency response. (c) Output from an MTI filter. [2]. 13

2.5 The Random Demodulator (RD) scheme [3]. . . . . . . . . . . . . . 22

2.6 Block diagram of the modulated wideband converter [4]. . . . . . . . 23

3.1 The relation between the closeness of the prior information and $P_{d}$. $\quad 34$

3.2 The relation between the closeness of the prior information and $P_{f a}$. 34

3.3 The relation between SNR and $P_{d}$. . . . . . . . . . . . . . 35

3.4 The relation between $P_{d}$ and $P_{f a}$. . . . . . . . . . . . . . 35

3.5 The relation between SNR of the original signal and SNR of the reconstructed signal. . . . . . . . . . . . . . . . 36

3.6 The relation between the SNR and $P_{d}$ for the original and the new target. 36

4.1 Flowchart illustrates the procedures of the proposed adaptive subNyquit rate algorithm using Haar wavelet coefficients. . . . . . . . . 44

4.2 Sampling using wavelet basis functions. . . . . . . . . . . . . . 45

4.3 Demonstration of the Haar wavelet coefficients selection. The coarse resolution bases are selected along the signal. The fine resolution bases are selected only within a window where there is target potential. . . 45

4.4 ROC curve using both Haar wavelet and Fourier bases $\left(P_{f a}=10^{-5}\right) . \quad 47$

5.1 ROC curve for a simulation of a single target in a homogeneous background using different basis functions $\left(P_{f a}=10^{-5}\right)$. . . . . . . . 62

5.2 ROC curve for a simulation of two targets in a homogeneous background using different basis functions $\left(P_{f a}=10^{-5}\right)$. . . . . . . . .

5.3 ROC curve for a simulation of a target in a clutter zone using different basis functions $\left(P_{f a}=10^{-5}\right) \ldots \ldots \ldots \ldots$ 
6.1 Probability of detection vs. probability of false alarm for SNR $=5 \mathrm{~dB}$, and three values of $K: K \max / 2, K \max , 2 K \max . \quad \ldots . . .$.

6.2 Probability of detection vs. probability of false alarm for SNR $=10$ $\mathrm{dB}$, and three values of $K: K \max / 2, K \max , 2 K \max . \quad \ldots \ldots$ 


\section{List of Tables}

4.1 Recovery statistics for different number of coefficients . . . . . . . . 49

4.2 Reconstruction performance for different number of coefficients . . . 49

5.1 Recovery statistics for different number of coefficients . . . . . . . . . 64

6.1 Support recovery guarantees for different measurement matrices . . . 69 


\title{
Nomenclature
}

\author{
Acronym Meaning \\ A-CAMP adaptive CAMP \\ ADC analog-to-digital converter (A/D) \\ ADT automatic detection and tracking \\ AWGN additive white Gaussian noise \\ BP basis pursuit \\ BPDN basis pursuit denoising \\ BPIC basis pursuit with inequality constraint \\ CA-CFAR cell average CFAR \\ CAMP complex approximation message passing \\ CFAR constant false alarm rate \\ CoSaMP compressive sampling matching pursuit \\ CPI coherent pulse interval \\ CS compressive sensing \\ DFT discrete Fourier transform \\ DMF decimated matched filter \\ DOST discrete orthogonal S-transform \\ DSP digital signal processing \\ FMCW Frequency modulated continuous wave \\ GPR ground penetrating radar




\begin{tabular}{|c|c|}
\hline IF & intermediate frequency \\
\hline LASSO & least absolute shrinkage and selection operation \\
\hline LCSF & localized cross spectral function \\
\hline MF & matched filter \\
\hline MMV & multiple measurement vectors \\
\hline MP & matching pursuit \\
\hline MRI & magnetic resonance imaging \\
\hline MSE & mean square error \\
\hline MTI & moving target detection \\
\hline MWC & modulated wideband converter \\
\hline NP-Hard & non-deterministic polynomial-time hard \\
\hline OMP & orthogonal matching pursuit \\
\hline OS-CFAR & order statistic CFAR \\
\hline PSD & power spectrum density \\
\hline$P_{d}$ & probability of detection \\
\hline$P_{f a}$ & probability of false alarm \\
\hline$P_{f t p}$ & probability of false target potential \\
\hline$P_{t p d}$ & probability of target potential detection \\
\hline $\mathrm{PRF}$ & pulse repetition frequency \\
\hline PRI & pulse repetition interval \\
\hline $\mathrm{RCS}$ & radar cross section \\
\hline $\mathrm{RD}$ & random demodulator \\
\hline $\mathrm{RF}$ & radio frequency \\
\hline RIP & restricted isometry property \\
\hline
\end{tabular}


ROC receiver operating characteristic

SAR synthetic aperture radar

SNR signal to noise ratio

STAP space-time adaptive processing

STT single target tracker

TC test cell

TWR through the wall radar

TWS track while scan

UWB ultra wide bandwidth 


\section{Chapter 1}

\section{Introduction}

\subsection{Efficient Sampling}

The Nyquist-Shannon sampling theorem has ruled the digital world for decades. The theorem, simply, emphasizes that the analog-to-digital converter (A/D), which is considered as the gateway to the digital world, must sample the signal at a rate two times greater than the signal bandwidth in order to avoid data loss. No one can deny that this theorem has had a great impact on signal processing techniques for ages. However, recently the demand for higher speeds is growing every day which implies a higher demand for increased signal acquisition and processing speeds and wider signal bandwidths (ultra wide bandwidth (UWB)). Consequently, that leads to a data deluge and requires higher rate A/Ds . For a certain limit, A/D advances cannot meet these demands. Moreover, it was observed that the high rate sampling stage is followed by a compression stage where most of these samples are discarded. The reason behind that is that real world signals seem to be in high-dimensional spaces. However, the useful data mostly lives in low-dimensional manifolds [5]. In other words, most signals can be represented in a sparse representation in a certain domain. For example, the sinusoidal signal is not sparse in the time domain. However, in the frequency domain, it is represented as spikes at the associated signal frequencies which is definitely a sparse representation.

Hence, a surge of research activity has been done to investigate how to deal with this data deluge by developing an efficient sampling approach that may alleviate these difficulties.

Signal sparsity was the foundation on which Candes, Romberg, and Tao [6] build 
their nonlinear sampling theorem. It demonstrates that a sparse signal can be recovered with very high probability from a number of measurements lower than the number of samples dictated by the Nyquist rate. This theorem was the door for a new signal processing field called compressive sensing (CS). The main goal of CS is the reduction in the required number of samples and the amount of data.

In this thesis, our focus is on the pulse radar signal. The expected received radar signal is a signal which includes shifted versions of the transmitted frequency modulated narrow rectangular pulse. Simply, a number of the received pulses expresses a number of targets in the scene and their time shifts express target range from the radar. The shift of the received frequency in each pulse expresses the target relative speed to the radar. The need for higher range resolution means shorter pulse width which consequently reflects a wider bandwidth. In some radar applications, UWB is needed not just for higher range precision, but also for some advantages that UWB offers such as the ability to penetrate through obstacles, low electromagnetic radiation and processing energy consumption, and high jamming immunity [7,8]. However, it will increase the system complexity.

Usually, the radar range is divided into a number of range cells (sometimes called range bins). In many radar applications, the expected number of targets is smaller than the number of range cells. Hence, the radar signal can be considered sparse in the time domain. Thus, the CS approach fits perfectly in this context.

\subsection{Problem Statement and Research Objective}

Since CS has been developed, many pieces of research have focused on it to demonstrate its validity to replace the classical sampling approach. Not surprisingly, the radar field got a good share of this research. Radar imaging takes the lion's share of this research such as in synthetic aperture radar (SAR) [9,10], through the wall radar (TWR) [11,12], and ground penetrating radar (GPR) $[13,14]$.

In the radar tracking problem, [15-17] introduce adaptive CS processing schemes using a particle filter to improve the performance of radar tracking.

CS would add improvements in many radar applications. However, some practical issues have to be addressed to be able to design a reliable CS based radar system. We can summarize the main points that play a crucial role in CS as follows.

1. Design of a practical implementation of a CS hardware for signal acquisition. 
2. Design of the CS measurement matrix.

3. The computational complexity of the signal reconstruction algorithm

The first point addresses the front end of the CS system which is the data acquisition step or the sampling process. The acquisition system should be designed efficiently to be simple, accurate and applicable. It is also preferable to use available low power components in the system design.

The second point addresses the measurement matrix design. The measurement matrix has a pivotal role in CS systems. It has a direct effect on both the system acquisition and the signal reconstruction steps. The design of the measurement matrix should be practically simple and should allow signal compression with minimum data loss. Moreover, the measurement matrix has to satisfy certain conditions such as the restricted isometry property and the non-coherence property (both will be discussed later in Sec. 2.2).

The third point addresses the reconstruction algorithm that should provide high signal estimation performance (i.e., the recovered signal should be almost equal to the original signal, $\hat{x} \simeq x$ ). However, the signal reconstruction step is the most time-

consuming part of the CS system. Hence, it is needed to be compatible with the application speed requirements (i.e., do not provide system delays).

\subsubsection{Hypothesis Statement}

We hypothesize that we can improve CS based pulse radar by designing a CS measurement matrix with time/frequency localization that allows adaptive sampling across the pulse repetition intervals using prior information. This approach is believed to reduce reconstruction computational complexity or even allow desired target parameter estimation without reconstruction. The approach is also believed to have an efficient practical implementation.

Since the measurement matrix plays a crucial role in both processes of signal acquisition and signal reconstruction, our hypothesis focuses on designing a suitable measurement matrix. We suggest some rules should be applied to the measurement matrix to ensure signal acquisition and reconstruction.

The first rule, the measurement matrix should allow practical implementation. That means that we have to consider the hardware limitations while designing the measurement matrix. 
The second rule, suitability to the signal nature. In our work, we consider a pulse radar signal with a constant carrier frequency. Hence, we believe that multiresolution analysis is a suitable approach to be considered due to its time/frequency localization properties. Moreover, the radar signal is near repetitive. This property can be exploited with the concept of prior information which showed a good potential in different applications.

The third rule, the design of the measurement matrix should consider the signal reconstruction complexity. Since the reconstruction process is the most timeconsuming process in compressive sensing, the measurement matrix should be carefully designed to allow low computational complexity in the reconstruction process to reduce the latency as much as possible.

The fourth rule, the measurement matrix should retain the important signal information. In our scenario, it should keep all target information such as time delay, Doppler frequency, and the echo amplitude.

\subsection{Contributions}

\subsubsection{CS Radar using Prior Information}

The contributions of Chapter 3 are:

- Introducing a new formula to improve the utilization of the prior information in CS pulse radar.

- Suggesting using the previously received pulses as prior information.

- Studying the feasibility of the proposed formula using ROC curves.

\subsubsection{Low-Latency Sub-Nyquist Sampling Algorithm}

The contributions of Chapter 4 are:

- Introducing a low-latency sub-Nyquist adaptive sampling algorithm based on Haar wavelets and Xampling [4] approach.

- Evaluating the proposed algorithm performance and comparing the results with the Fourier coefficients Xampling approach. 


\subsubsection{Frequency and Phase Analysis using S-transform}

The contributions of Chapter 5 are:

- Introducing an adaptive CS based algorithm using S-transform basis functions to allow target range and Doppler information extraction in a low-latency processing time.

- Introducing CS moving target indicator (CS-MTI) approach.

- Evaluating the algorithm performance and comparing the results with the competing approaches and classical Nyquist rate sampling approach.

\subsubsection{Recovery Guarantees for the S-transform matrix}

The contributions of Chapter 6 are:

- A mathematical proof for the recovery guarantees for the proposed S-transform algorithm discussed in Chapter 5.

- Deriving an upper bound on the number of detectable targets.

\subsection{Publications for this Dissertation}

The work in this dissertation was published in three conference papers. The details are as follows.

- Assem M. Assem and Richard M. Dansereau, "Compressive Sensing in Pulse Radar using Prior Information", $4^{\text {th }}$ International Workshop on Compressed Sensing Theory and its Applications to Radar, Sonar and Remote Sensing (CoSeRa), 19-22 September 2016, Aachen, Germany.

- Assem M. Assem, Richard M. Dansereau, and Fathy M. Ahmed, "Adaptive SubNyquist Sampling Based on Haar Wavelet and Compressive Sensing in Pulsed Radar", $4^{\text {th }}$ International Workshop on Compressed Sensing Theory and its Applications to Radar, Sonar and Remote Sensing (CoSeRa), 19-22 September 2016, Aachen, Germany. 
- Assem M. Assem, Richard M. Dansereau, "Compressive Sensing using Stransform in Pulse Radar", TSP 2017 40th International Conference Telecommunications and Signal Processing. 5-7 July 2017, Barcelona, Spain.

\subsection{Dissertation Organization}

The remainder of the dissertation is organized as follows:

Chapter 2 introduces a brief description of the radar function and radar subsystems and an introduction to CS theory. We also discuss the effect of CS on the detection performance and radar detector functions. Moreover, we discuss in more detail two CS sampling process techniques, the random demodulator (RD) and the modulated wide-band converter (MWC).

Chapter 3 discusses the pulse radar signal recovery problem in CS. We introduce a new formula to deal with the prior information in pulsed radar along with an idea of exploiting the previously received signal to be used as prior information for the current signal in the new proposed reconstruction optimization formula. The performance of the compressive sensing reconstruction and detection are analyzed in different scenarios by means of ROC curves.

Chapter 4 focuses on the problem of compressive sensing sampling process for a pulse radar signal corrupted by additive white Gaussian noise. We propose an algorithm using the simple Haar wavelet bases to adaptively sample the signal at a sub-Nyquist rate based on compressive sensing framework. The proposed algorithm uses the previously received pulse interval as prior information for the present interval. Based on this information, the algorithm is able to sample the current interval at a low resolution and focus only on the target potential segments at high resolution. The target range detection performance of the proposed algorithm is evaluated using receiver operating characteristics (ROC) curves for different values of signal to noise ratio (SNR). Moreover, the reconstruction complexity is also evaluated using reconstruction time as a measure. An evaluation is carried out using a different number of samples and compared to competing approaches.

Using the Haar wavelet basis provides target range detection using simple system design and implementation and also faster reconstruction. However, it does not provide sufficient phase information needed for target speed extraction. 
Chapter 5 introduces a new algorithm that exploits the S-transform basis functions properties to allow extracting the target Doppler information. This chapter paves the way to build a low latency compressive sensing sub-Nyquist coherent pulse radar receiver capable of detecting the required target parameters which include target range (represented by the pulse time delay) and target speed (represented by its Doppler frequency).

Chapter 6 discusses compressive sensing from a mathematical point of view. A mathematical proof for the recovery guarantees for the proposed S-transform algorithm discussed in Chapter 5 is introduced. The main aim of this chapter is to derive an upper bound on the number of detectable targets in the presence of additive noise.

Chapter 7 represents a conclusion for this dissertation and studies the limitations of the proposed algorithms, Haar wavelet algorithm and S-transform algorithm. Moreover, our plans for the current work extension and implementation and the future work are presented in this chapter. 


\section{Chapter 2}

\section{Radar and Compressive Sensing Concepts}

In this chapter, we discuss briefly the concepts behind radar and the function of radar subsystems. Moreover, we introduce a background for compressive sensing theory and how it affects radar performance if used instead of classical radar sampling techniques. Furthermore, we discuss two techniques that can be implemented to achieve efficient sampling using CS.

\subsection{Radar In Brief}

Radar is an electromagnetic sensor. The main goal of radar is detecting the desired object (target) characteristics such as distance from the radar, relative speed, shape, type, etc.

A Radar transmitter radiates electromagnetic energy (signal) into space by means of the radar antenna. A portion of the propagated signal hits objects in space and scatters in many directions. The received signal is amplified and processed by the radar receiver to provide a decision for the target presence and extract the required target parameters [1].

A common type of the waveform used in radars is a series of relatively narrow rectangular pulses with pulse duration $\delta$ followed by a silent period to wait for the returned target echo to be received. This pulse is repeated periodically every time period $T$ called the pulse repetition interval (PRI). The pulse repetition frequency (PRF) $f_{r}$ is equal to the reciprocal of the PRI. Each PRI is evenly divided into a number of range cells. The time delay $t_{d}$ of the echo is directly related to the target 


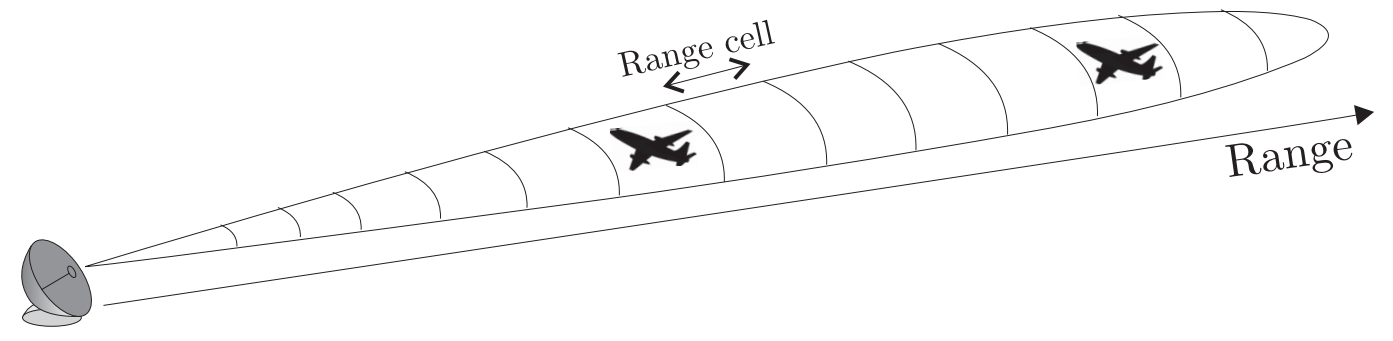

Figure 2.1: Example of air surveillance radar.

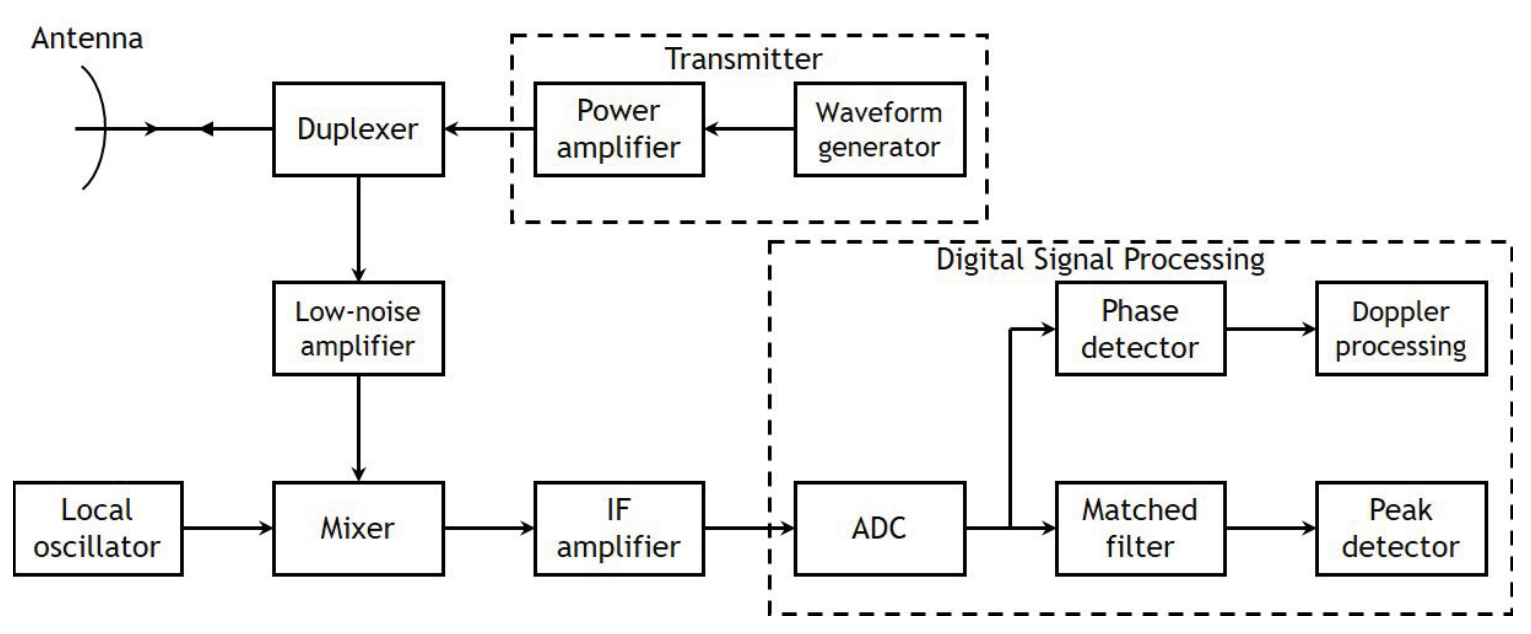

Figure 2.2: Block diagram of the basic radar subsystems [1]

range (distance from the radar) $R$ which can be calculated simply as [1]

$$
R=\frac{c t_{d}}{2}
$$

where $c$ is the speed of light in space $\simeq 3 * 10^{8} \mathrm{~m} / \mathrm{s}$. The factor of 2 comes from the round trip of the signal from and back to the radar. Figure 2.1 shows an example of air surveillance that demonstrate how the radar range is divided into range cells. This thesis mainly focuses on the described type of radar, which is called pulse (or pulsed) radar. 


\subsubsection{Radar Structure}

The basic subsystems usually found in radar are shown in Fig. 2.2. We can summarize their functions as follows [1]:

Transmitter: generate the waveform suitable for the radar's assigned function. In the case of pulse radar, radar transmission is limited to pulse duration and when the pulse is off, the transmitter is off.

Duplexer: controls the signal direction, i.e., close the reception path while transmitting to protect the receiver from burning by the high energy. Then, swap the direction during reception. That allows using a single antenna for transmission and reception.

Low-noise amplifier: amplifies the weak signal to a level to be detected.

Mixer: mixes the radio frequency $(\mathrm{RF})$ received signal with the local oscillator reference signal to produce the intermediate frequency (IF) signal $x(t)$.

Analog to digital converter (ADC): sample the received analog signal $x(t)$ at the Nyquist rate $f_{N}$ equal to the band width of the transmitted pulse $B_{W}$. The output of the ADC is a sampled version $x[n], 0<n<N$, where $N=T B_{W}$. Hence, the ADC is considered as the first step and the gateway of the digital signal processing stage.

Digital signal processing (DSP): the mission of the DSP is to suppress the noise and extract the useful target parameters. DSP consists of some basic stages that can be summarized as follows.

\section{Matched filter (MF)}

The matched filter (MF) can be considered the optimal linear filter to maximize the signal to noise ratio (SNR) in the presence of additive stochastic noise [1]. Since the transmitted pulse $p(t)$ is known, it is used as a template to be correlated with the received signal $x(t)$. That is done by the convolution process $x[n] * h^{*}[-n]$, where $h^{*}[-n]$ is the reverse of the conjugate of the sampled version of the transmitted pulse.

The MF is considered optimal in case that the received signal is identical to the transmitted one. That occurs only in the case of fixed clutter ( in most cases, clutters are not desirable). However, this is not the case of moving target echo that has a frequency shift resulting from the Doppler effect, which will be discussed later. This problem can be solved by compensating the Doppler frequency or using a bank of filters for different frequencies values. 


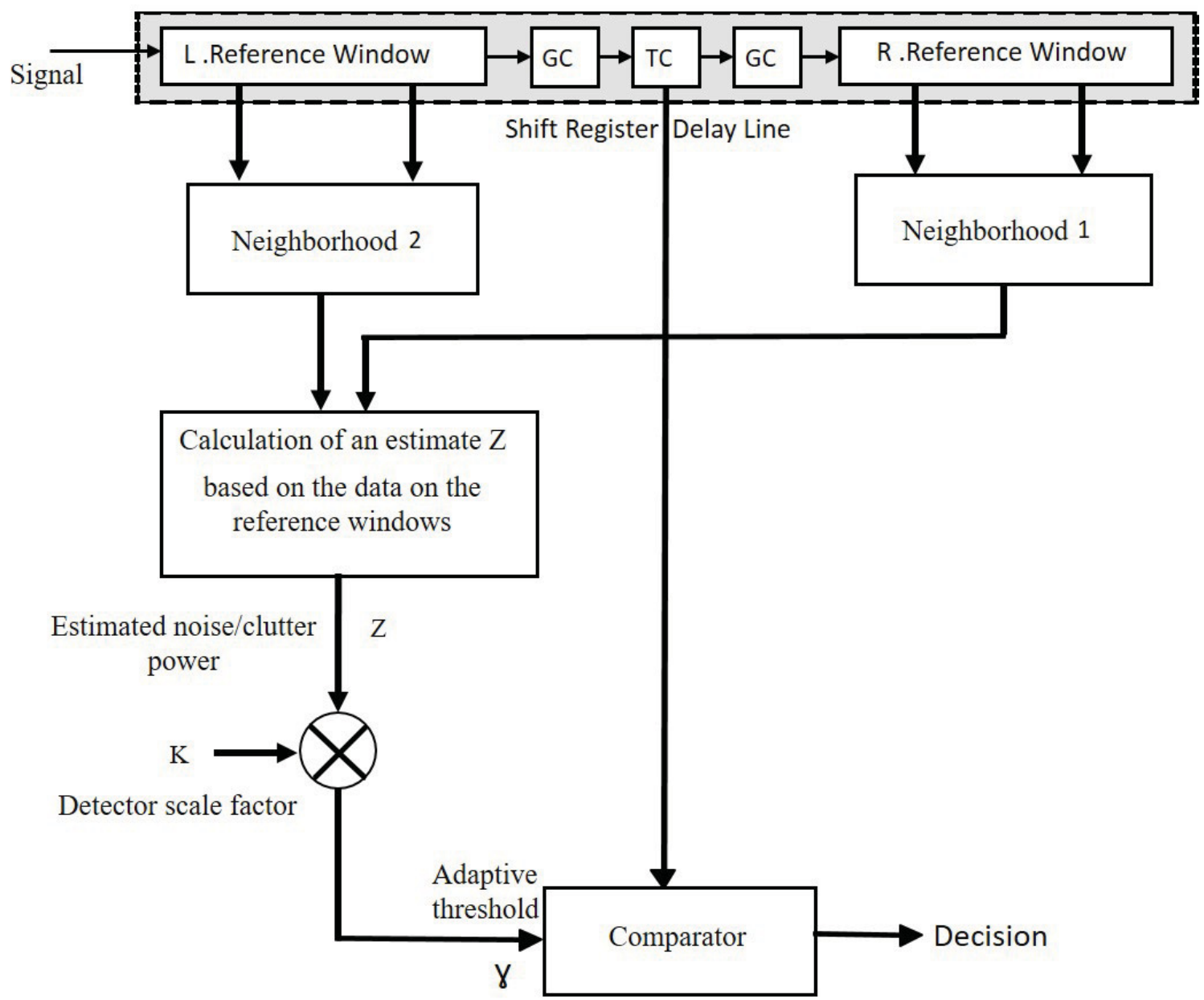

Figure 2.3: The central operation of the CFAR processor

\section{Peak detection}

Usually, the target is detected as a peak. The time delay of this peak provides the range information as in (2.1). The amplitude of the peak is related to the target's radar cross section (RCS), dispersion attenuation, and other propagation factors. In order to avoid false alarms, the peaks are detected based on a threshold which can be set as a fixed threshold or created based on certain criterion such as fixing the probability of false alarm $P_{f a}$ as in constant false alarm rate (CFAR) processor [1], which dynamically determines an adaptive threshold by estimating the local background noise/clutter power surrounding the test cell (TC) and multiplying this estimate by a scaling constant to maintain the designated $P_{f a}$ as shown in Fig. 2.3. The detection 
criterion for CFAR is expressed as

$$
x[n] \underset{H_{0}}{\stackrel{H_{1}}{\gtrless}} \gamma[n]
$$

where $H_{1}$ and $H_{0}$ represent the hypothesis of target presence and absence, respectively. $\gamma$ is the adaptive threshold created by CFAR algorithm.

\section{Doppler processing}

The frequency of the returned echo of a moving target is shifted, compared to the reference frequency. This frequency shift is called the Doppler frequency [18]. The Doppler frequency value is related directly to the target relative velocity to the radar and is given as [1]

$$
f_{d}=\frac{2 v_{r}}{\lambda} \cos \alpha
$$

where $v_{r}$ is the target radial speed, $\lambda$ is the wavelength, and $\alpha$ represents the angle between direction of the reflected signal and the target flight direction.

Since the output of the phase detector provides the phase shift between the received signal and the reference signal, then the value of the phase change will be modulated by the Doppler frequency. Hence, the Doppler frequency for each target can be detected by applying a p-point discrete Fourier transform (DFT) for each discrete time index $n$ along the PRI.

\section{Moving target indicator (MTI)}

Fixed (stationary) and slow-moving objects echoes are unwanted echoes for many radar applications (such as military surveillance radars and airport surveillance radars). In most cases, these echoes are classified as clutter. This type of clutter power spectrum density (PSD) can be expressed as [2]

$$
S_{c}=\frac{P_{c}}{T \sigma_{f} \sqrt{2 \pi}} \sum_{k=-\infty}^{\infty} \exp \left[\frac{-(f-k / T)^{2}}{2 \sigma_{f}^{2}}\right]
$$

where $T$ is the PRI (reciprocal of the radar PRF), $P_{c}$ is the clutter power and $\sigma_{f}$ is the clutter spectral spreading parameter.

Obviously, the clutter PSD is concentrated around zero and multiple integers of the radar $\mathrm{PRF} f_{r}$. The goal of MTI is to suppress the clutter (stationary targets) and 

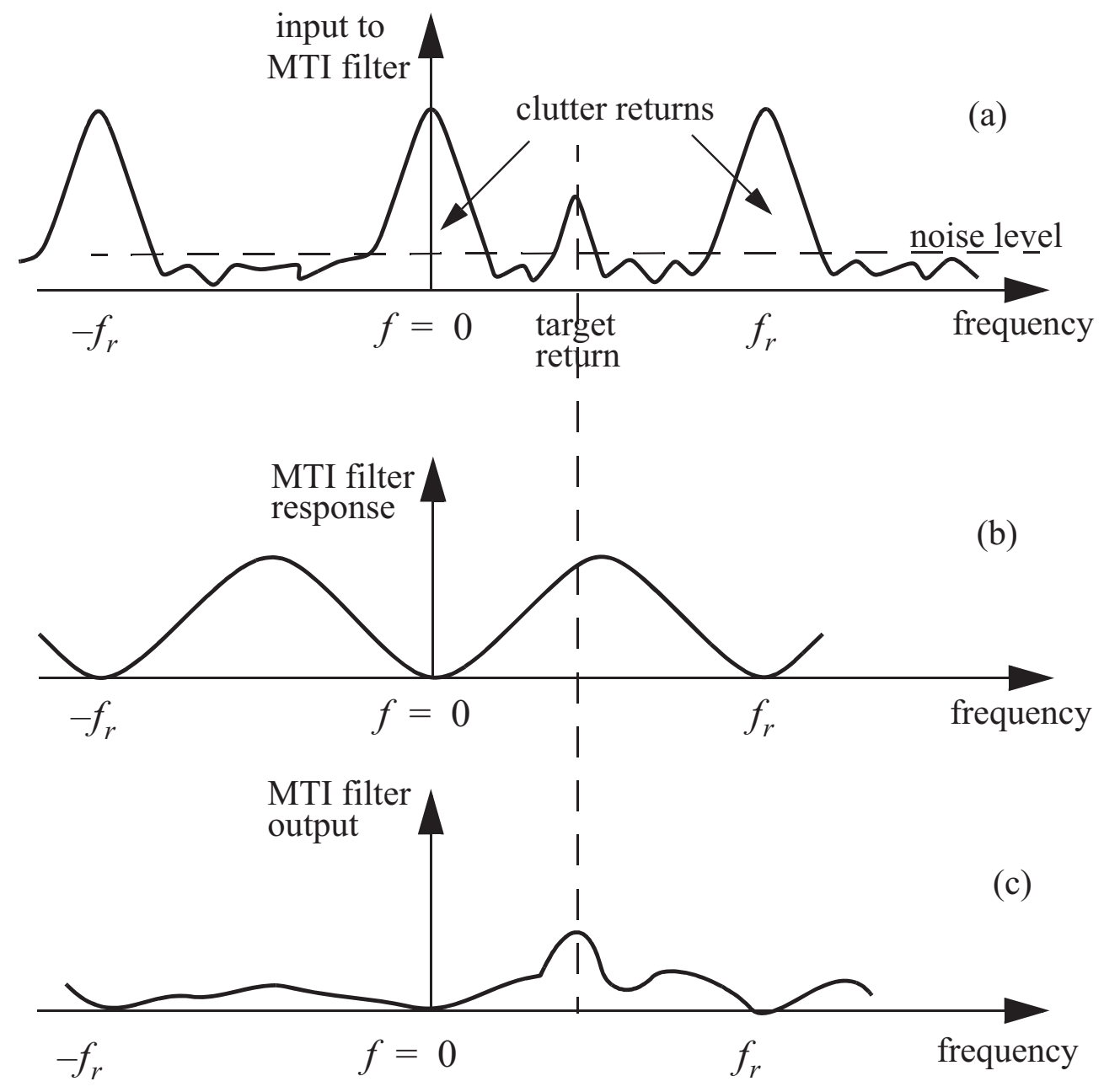

Figure 2.4: (a) Typical radar return PSD when clutter and target are present. (b) MTI filter frequency response. (c) Output from an MTI filter. [2].

let moving targets pass through with negligible effect. The simplest way to achieve that is to design a band-stop filter at DC $(f=0)$ and at all multiples of the radar PRF.

Figure 2.4 shows the MTI filter response for target and clutter. However, that means targets with Doppler frequencies $f_{d}=n f_{r}$ will be suppressed too. The speeds associated with these Doppler frequencies $\left(n f_{r}\right)$ are called blind speed, $v_{b l i n d}=n *$ $f_{r} \lambda / 2$, where $\lambda$ is the wavelength and $n \geq 0$. This problem can be avoided by using multiple PRF scheme. 


\subsubsection{Radar Bandwidth}

Radar bandwidth $B_{W}$ for pulse radar is usually calculated as the reciprocal of the pulse duration, $B_{W}=1 / \delta$. Bandwidth plays a crucial role in radar capabilities. For instance, wider bandwidth means higher range resolution.

Many of the ultra wide band (UWB) features have long been appreciated as a key advantage for radar such as penetrating through obstacles, high precision ranging, low electromagnetic radiation, low processing energy consumption, and immunity to jamming $[7,8]$.

However, UWB radar faces some difficulties. It requires transmission of wide bandwidth during a short observation time. Consequently, it requires a wide bandwidth receiver responsible for acquiring the received signal at a very high rate ADC, which, in most cases, causes unaffordable hardware complexity.

Therefore, considerable research interests have been thought to find a new approach to acquiring the signal at a rate lower than Nyquist rate (or so-called subNyquist rate) to alleviate these difficulties and reduce the system complexity $[6,19]$.

\subsection{Compressive Sensing Background}

The Fourier transform is considered one of the most important tools in signal analysis. However, it provides frequency information while hiding the time information of the signal.

A fundamental signal decomposition approach, called Gabor's approach, was a solution for this problem, [20]. Later, Hilbert space frame, in which Gabor's approach and the Fourier transform are special cases, is introduced to deal with time frequency analysis using overcomplete (redundant) set of vectors [21]. In other words, the number of vectors in the frame exceeds the dimension of the space. Thus, each vector in the space does not have a unique representation with respect to the frame. This redundancy offers greater design flexibility and robustness [5].

Mathematically, a family of vectors $\left(\varphi_{i}\right)_{i=1}^{N}$ in Hilbert space $\mathscr{H}^{N}$ is called a frame for $\mathscr{H}^{N}$ if there exist constants $0<A<B<\infty$ such that

$$
A\|x\|^{2} \leq \sum_{i=1}^{N}\left|\left\langle x, \varphi_{i}\right\rangle\right|^{2} \leq B\|x\|^{2}
$$

for all $x \in \mathscr{H}^{N}[5]$. 
The set of vectors $\left(\varphi_{i}\right)_{i=1}^{N}$ and the adapted system $\left(\tilde{\varphi}_{i}\right)_{i=1}^{N}$ should be chosen carefully and suitable to the nature of the data $x$ to achieve the mapping

$$
x \mapsto\left(\left\langle x, \varphi_{i}\right\rangle\right) \mapsto \sum_{i=1}^{N}\left\langle x, \varphi_{i}\right\rangle \tilde{\varphi}_{i} \approx x .
$$

Using carefully chosen frames, the data can be represented in a sparse representation such that the number of non-zero entries are much less than the number of zero entries. Let us consider that signal $x \in \mathscr{H}^{N}$ is $k$-sparse [5], i.e,

$$
\|x\|_{0}:=\sum_{i=1}^{N} 1_{\left\{x_{i} \neq 0\right\}}(x)=k \ll N .
$$

Then $\left(\varphi_{i}\right)_{i=1}^{N}$ can be chosen such that the expansion of the data $x$

$$
x=\sum_{i=1}^{N} c_{i} \varphi_{i}
$$

satisfies that the sequence $\left(c_{i}\right)_{i=1}^{N}$ is $k$ sparse.

In recent years, compressive sensing (CS) has come to light as a new signal processing paradigm $[6,19]$. CS exploits signal sparsity characteristics to compress the signal using a number of samples much lower than the Nyquist rate. The fundamental goal in CS is to acquire the signal using a small number of measurements $y$ where

$$
y=\Phi x
$$

and recover/reconstruct the sparse signal $x$ without data loss. In the CS field, the $M \times N$ matrix $\Phi$ is called the measurement matrix.

It is worth mentioning that signal $x$ is directly represented in a sparse representation in some applications. In other applications, the signal might not have the required sparse nature. However, it can be sparse in some other basis or dictionary $\Psi$. Then the measurement $y$ will be

$$
y=\Phi x=\Phi \Psi s
$$

where $s$ is the sparse representation of $x$ with respect to basis $\Psi$. For instance, a pure sine wave is not sparse in the time domain. However, in the frequency domain it is.

The geometry of the measurement matrix plays a central role in the CS signal 
compression and reconstruction phases. The dimension $M$ in the measurement matrix defines the number of measurements $y$ which also corresponds to the number of samples in regular sampling schemes. This leads to the question of how many $M$ measurements are required to perfectly reconstruct $x$ from $y$ ? If $M \geq N$ and $\Phi$ has full rank, then the problem is determined or over-determined which can be solved uniquely. In the case of $M<N$, then the problem is under-determined. Which means that it has infinitely many solutions. Fortunately, we are looking for the $k$-sparse $x$ solution only, i.e, where $x$ has only very few non-zero components. Actually, that is the key of CS.

In recent years, there has been a great deal of work in recovering the sparse support of a signal $x$.

The recovery of such a signal can be perfectly achieved by finding the sparsest solution by solving an $\ell_{0}$ norm minimization problem. Where $\ell_{p}$ norm of vector $x$ is defined as

$$
\left(\|x\|_{p}\right)^{p}=\sum_{i=1}^{n}\left|x_{i}\right|^{p} .
$$

Then the optimization problem can be expressed as

$$
\hat{x}_{0}=\underset{x \in \mathscr{H}^{M}}{\arg \min }\|x\|_{0} \quad \text { s.t } \quad y=\Phi x
$$

where $\hat{x}_{0}$ is the sparsest solution that satisfies $y=\Phi \hat{x}_{0}$.

The following theorem provides the minimum and satisfactory condition for this problem.

Theorem 1. [5] An arbitrary $k$-sparse signal $x$ can be uniquely recovered from $y=$ $\Phi x$ as a solution to an $\ell_{0}$ minimization problem provided

$$
k<\frac{1}{2}\left(1+\mu_{\Phi}^{-1}\right)
$$

where $\mu_{\Phi}$ is the worst-case coherence which expresses the measure of the similarity between different frame elements [5], and can be defined as

$$
\mu_{\Phi}=\max _{\substack{i, j \in\{1, \ldots, m\} \\ i \neq j}} \frac{\left|\left\langle\varphi_{i}, \varphi_{j}\right\rangle\right|}{\left\|\varphi_{i}\right\|\left\|\varphi_{j}\right\|} .
$$


Unfortunately, $\ell_{0}$ is a highly non-convex optimization problem. It requires an exhaustive combinatorial search over all possible solutions of the frame elements. That makes the complexity exponential to $M$ which leads to a non-deterministic polynomial-time hard (NP-Hard) problem.

However, some research efforts have been made to find practical alternatives to $\ell_{0}$. The upshot of these efforts is that some convex optimization-based methods and greedy algorithms can provide good results for this problem. We will discuss both of these methods briefly.

Since the major problem of $\ell_{0}$ norm minimization is that it is a non-convex optimization, one of the solutions is to approximate it with a convex problem such as $\ell_{1}$ norm which is a linear optimization problem and can be expressed as

$$
\hat{x}_{1}=\underset{x \in \mathscr{H}^{M}}{\arg \min }\|x\|_{1} \quad \text { s.t } \quad y=\Phi x
$$

where $\hat{x}_{1}$ is the sparsest solution that satisfy $y=\Phi \hat{x}_{1}$.

This problem is called basis pursuit (BP) [22]. Surprisingly, the minimum and satisfactory condition for the $\ell_{1}$ problem is the same as the condition of the $\ell_{0}$ problem in (2.13). However, because this is only a sufficient condition, the solution of each problem is not guaranteed to be the same [5].

As an alternative to $\ell_{1}$ relaxation, [23] introduced the FOCUSS algorithm that employs $\ell_{p}$ norm instead of $\ell_{0}$ for $0<p<1$. Although this problem is not non-convex, FOCUSS provides an iterative algorithm to solve the optimization problem.

The other way to solve the CS problem is through greedy algorithms, such as matching pursuit (MP) [24] and orthogonal matching pursuit (OMP) [25]. For sufficiently small worst case coherence $\mu_{\Phi}$, [26] shows that OMP can recover $k$-sparse signal support with high probability. Moreover, these results are further studied and improved in [27] to show that the signal can be recovered using only $O(k \log N)$ measurements. Again, the condition in (2.13) also works for the OMP as a minimum and satisfactory condition [5]. That leads us to the result that as long as $k=O\left(\mu_{\Phi}^{-1}\right)$, an underdetermined system of equation $y=\Phi x$ for a $k$-sparse signal can be solved in polynomial time [5].

An improved OMP approach called compressive sensing matching pursuit (CoSaMP) was introduced in [28]. CoSaMP provides better stability and robustness results. It is robust against noise and offers a uniform performance, which depends only on the sampling operator property and the signal sparsity not on the 
size of the non-zero signal coefficients [28]. Moreover, it requires minimal storage and computations and works with a minimal number of observations.

To consider the measurement noise $n$ added to the signal, a small modification can be made to (2.15) to express the optimization problem for a noisy case to be expressed as [5]

$$
\hat{x}_{1}=\underset{x \in \mathscr{H}^{M}}{\arg \min }\|x\|_{1} \quad \text { s.t } \quad\|y-\Phi x\|_{2} \leq \epsilon
$$

where $\epsilon=\|n\|_{2}$, i.e., it is typically chosen to be equal to the noise magnitude. This modified version is called basis pursuit with inequality constraint (BPIC) [5]. Regarding OMP, the same algorithm should work without modification except the value of $\epsilon=\|n\|$ instead of 0 in the former case.

It is worthy to note that in the noisy case, for both methods, the recovered signal will be considered an estimation for the sparse signal.

This noisy model in (2.16) is considered as a deterministic model. If we wish to deal with stochastic noise such as additive white Gaussian noise (AWGN), then (2.16) can be modified as

$$
\hat{x}=\underset{x \in \mathscr{H} M}{\arg \min } \frac{1}{2}\|y-\Phi x\|_{2}^{2}+\lambda\|x\|_{1}
$$

where $\lambda$ is a regularization parameter that controls the trade off between error and the sparsity of the solution and is chosen based on empirical considerations. In Sec. 2.4, we will discuss some available techniques to choose the value of $\lambda$. This optimization problem is called basis pursuit denoising (BPDN) [22] or least absolute shrinkage and selection operation (LASSO) [5].

Back to the general CS problem, we have addressed the methods of the sparse signal recovery from a small number of measurements $y$ as in (2.9) or (2.10). Now, we will discuss the sufficient conditions required to reconstruct a stable measurement matrix $\Phi$. Simply, there are two sufficient conditions $[6,19]$.

(I) satisfying the restricted isometry property (RIP). RIP can be defined as

Definition 1. [5] (Restricted Isometry Property (RIP))

A unit norm frame $\Phi=\left(\varphi_{i}\right)_{i=1}^{M}$ in $\mathscr{H}^{N}$ is said to have the RIP of order $k$ with parameter $\delta_{k} \in(0,1)$ if for every $k$-sparse $x$, the following inequalities hold:

$$
\left(1-\delta_{k}\right)\|x\|_{2}^{2} \leq\|\Phi\|_{2}^{2} \leq\left(1+\delta_{k}\right)\|x\|_{2}^{2}
$$


(II) the incoherence condition between the rows of $\Phi$ and the columns of $\Psi$.

These two conditions can be simply met by choosing $\Phi$ as a random matrix. Hence, an iid Gaussian matrix can satisfy the RIP with high probability if $m \geq$ $c k \log (N / k) \ll N$ with $c$ a small constant [6,19,29]. Choosing $\Phi$ as an iid Gaussian matrix also provides the universality property. i.e., regardless the choice of $\Psi, \Phi$ will be incoherent with very high probability. The complete random matrix also provides a very important privilege for CS; no previous information will be needed from the signal of interest other than sparsity.

We can summarize the main compressive sensing hallmarks as follows:

1. Breaking the Nyquist rate ( replacing $N$ samples by $k \log (N / k))$.

2. Directly acquire the compressed data.

3. Universality (can be used with any compressible signal).

4. No signal information needed ( other than sparsity) .

\subsection{CS Radar Detection Performance}

Since compressive sensing has come to light as a new signal processing paradigm, a considerable number of researchers demonstrated the CS concept validity in radar signal processing. The effect of applying CS on radar detection performance is one of the most critical points in radar.

In [30], the radar detection performance has been addressed considering new parameters that appear due to interleaving CS with classical radar detection schemes, such as the reconstruction threshold $\epsilon$, compression factor $\delta_{C}$, and the target sparseness $k$. The measurement matrix was created as a sub-matrix selected from the discrete Fourier transform (DFT) matrix. In this case, the signal is sparse in the time domain directly. Hence, the matrix $\Psi$ in $(2.10)$ is set to be identity matrix, $I_{N}$. To compare between the classical radar and CS radar, a classical matched filter (MF) and a decimated matched filter (DMF) have been simulated. The DMF is simulated in the same way as the MF but using lower number of samples.

The simulation in [30] was carried out using several sets of parameters $\epsilon$ (appears in (2.16)), SNR, $M, N$ and $k$ in different target scenarios. Moreover, two detection criteria have been used. The first one is to consider any non-zero coefficient of the 
received signal as a hit without using further processing. The second is done by applying a detector after the signal recovery step and take the decision after the detector.

The results for all scenarios show that detection performance for CS estimation is close to the classical approaches. The results emphasize that choice of the reconstruction threshold value is substantial in CS detection behaviour. Moreover, it was demonstrated in [30] that coherent integration can be useful even after CS reconstruction and that it improves SNR significantly.

Hence, CS can be used in radar applications to reduce the radar system complexity while we have to design the CS parameters carefully to achieve the desired detection performance.

\subsection{Radar Detectors using Compressive Sensing}

As we have discussed in Sec. 2.2, there are many approaches for sparse signal recovery from compressed sensing measurements. LASSO optimization problem in (2.17) is widely used for signal recovery while the signal is contaminated with AWGN, as it is the case of the received radar signals. However, it is not easy, until now, to tune the regularization parameter $\lambda$ in (2.17) [31]. That is because the choice of $\lambda$ depends on the noise level and signal sparsity (i.e., number of non-zero coefficients in the signal), and, mostly, both are unknown in prior.

One of the successful CS recovery algorithms solving LASSO called complex approximation message passing (CAMP) was introduced in [31]. CAMP algorithm user specified threshold $\tau_{C A M P}$ was shown to have a one-to-one relationship with the regularization parameter $\lambda$ in LASSO [31]. The optimum value of $\tau_{C A M P}$ is the value that achieves minimum mean square error (MSE) between the reconstructed (estimated) signal and the original signal $x$. It is shown in [32] that by controlling the value of $\tau_{C A M P}$ adaptively, one can achieve a good signal estimation without any prior knowledge neither for the signal nor the noise variance. That was achieved by so-called adaptive CAMP (A-CAMP).

Since A-CAMP calculates an estimate of the output noise in each iteration, it has a direct effect on the recovery SNR and maximizes SNR by minimizing the output noise variance.

The radar detection criterion is preferably done by fixing the probability of false 
alarm $P_{f a}$ which can be achieved by using the CFAR algorithm as mentioned in Sec. 2.1.1. Three CS CAMP architectures are introduced in [32]. The first one uses A-CAMP as a CFAR detector inherently by exploiting the parameter $\tau_{C A M P}$ as an adaptive controller for the detection threshold at the desired $P_{f a}$. However, the simulation results were not satisfactory. Hence, the authors recommended using a second detector after the reconstruction process to enhance $P_{d}$ as proposed in the second approach.

In the second architecture, A-CAMP recovers the signal and maximizes the SNR, then it is followed by the second detector. The classical CFAR processor was suggested to be used as a second detector to control the constant $P_{f a}$.

In the third architecture, instead of using the A-CAMP final output to feed the second detector, the output of each iteration is used. This slight change allows better detection of the low SNR targets that might be lost at the optimum output of ACAMP.

An evaluation for $P_{d}$ in different scenarios of targets is presented in [33]. The evaluation was carried out using two of the most famous CFAR detectors, the cell average CFAR (CA-CFAR) and order statistic CFAR (OS-CFAR). The results confirmed the suggestion of using CFAR as a second detector instead of using A-CAMP to maintain constant $P_{f a}$.

\subsection{Sampling Process in CS}

Due to the potential of CS to reduce system complexity by allowing a lower rate ADC, a surge of research activity has been done to investigate how to exploit CS advantages in different applications. However, few of them address how the sampling process can be performed using CS. The random demodulator (RD), introduced in [3] and further discussed in [34], and Xampling approach, introduced in [4,35], are some successful examples. We will discuss these two approaches in this section.

\section{Random Demodulator (RD)}

The random demodulator $(\mathrm{RD})$, introduced in [3] was one of the first successful trials to deal with CS sampling process. RD utilizes the available low power components to acquire the higher band limited signals exploiting CS approach. As shown in 


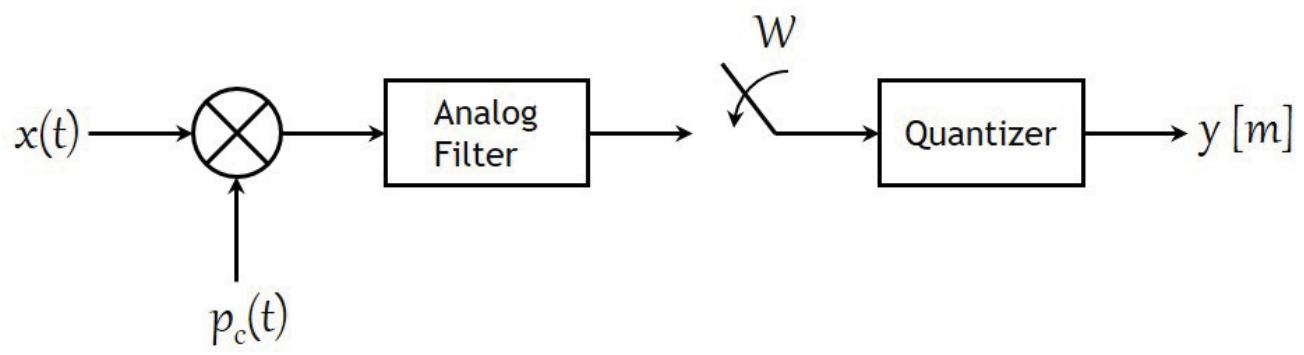

Figure 2.5: The Random Demodulator (RD) scheme [3].

Fig. 2.5, a pseudo-random number generator is used to create a high rate pseudo random sequence $P_{c}(t)$ of \pm 1 at a rate equal to or exceeding the Nyquist rate of the input signal. The received signal is demodulated with $P_{c}(t)$ such that the signal frequency component is smeared and not destroyed by the next stage which is a low pass filter that acts as an integrator. Afterwards, the compressed measurements $y$ are generated using a low rate sampler at rate $W$ where $W \ll$ Nyquist rate. Obviously, the compressed signal $y$ has much lower samples than the Nyquist rate of the original signal and can be represented in the form $y=\Phi x$, where the measurement matrix $\Phi$ is created from the pseudo random sequence $P_{c}(t)$. Hence, the original signal can be recovered using CS recovery algorithms discussed in Sec. 2.2.

However, the expense of the reduction of the number of samples has been paid by the digital reconstruction process, i.e., the burden on the analog processing that should be done by the ADC is switched to the digital reconstruction process.

\section{Xampling and The Modulated Wideband Converter (MWC)}

Another CS based methodology of sampling by a sub-Nyquist rate has been introduced in [4] and [35], called Xampling. It performs data compression in the sampling stage implicitly. Xampling methodology proposed four main principles that should be satisfied in the sub-Nyquist rate design, i) the ability to capture a broad signal model, ii) low sampling rate, iii) efficient analog and digital implementation and iv) low rate baseband processing [4]. As an application for Xampling, the modulated wideband converter (MWC) is introduced in [4]. It is demonstrated that it satisfies the four Xampling principles. As shown in Fig. 2.6, the design of the MWC is based 


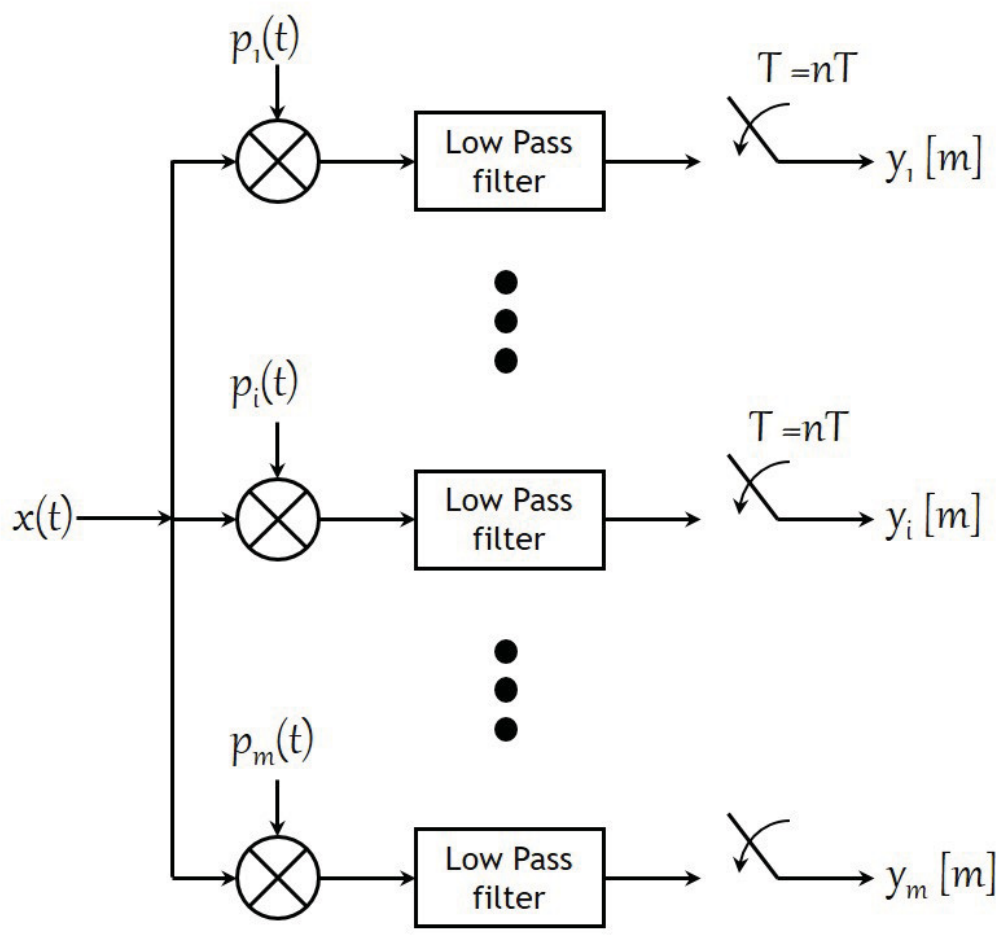

Figure 2.6: Block diagram of the modulated wideband converter [4].

on branching the input analog signal into $m$ channels. The input signal in each channel is multiplied by a periodic waveform. The signal periodicity differs in each channel to span a wide frequency band. The output of each channel is low pass filtered and then sampled at a lower rate.

The main concept of the MWC is based on scrambling the spectrum of the input signal by mixing it with the periodic signal, such that the frequencies left after the low pass filtering process comprise a combination of the spectral content of the entire signal. The signal recovery can be carried out by using regular CS recovery algorithms.

Exploiting Xampling framework features, [36] discussed how the radar signal can be acquired at a sub-Nyquist rate and how target detection can be achieved. It was demonstrated that the Fourier series coefficients contain the required target parameter as follows. Consider a received pulse train of $P$ pulses

$$
x(t)=\sum_{p=0}^{P} \sum_{\ell=0}^{L-1} a_{\ell} h\left(t-t_{\ell}-p T\right), \quad a_{\ell} \in \mathbb{C}, t_{\ell} \in[0, T)
$$

where $L$ is the number of targets and $h\left(t-t_{\ell}-n T\right)$ is the received echo for target $\ell$ 
at delay $t_{\ell}$ in period $n$ with amplitude $a_{\ell}$. The periodic signal $x_{p}(t)$ is defined as

$$
x_{p}(t)=\sum_{l=0}^{L-1} a_{l} h\left(t-t_{l}\right)
$$

and can be expressed by its Fourier series

$$
x_{p}(t)=\sum_{k \in \mathbb{Z}} c_{p}[k] e^{j 2 \pi k t / T}
$$

where

$$
c_{p}[k]=\frac{1}{T} \int_{0}^{T} x_{p}(t) e^{-j 2 \pi k t / T} d t .
$$

Thus, MWC can be used to acquire the radar signal by choosing the periodic signal as the Fourier bases. Then, the signal parameters can be recovered using fewer measurements by projecting the signal onto a subset of its Fourier series coefficients. By using a simple low pass filter followed by sampling at twice its cut-off frequency, a set of consecutive coefficients can be obtained from the discrete Fourier transform (DFT) of the samples. However, [37] shows that selecting distributed coefficients over the signal spectrum results in a better signal recovery performance. On the other hand, [38] demonstrated that at least two consecutive Fourier coefficients should be chosen. To satisfy these two constraints, sets of consecutive coefficients can be chosen from different frequency bands [38]. The signal recovery can be done by solving

$$
c_{p}=A x_{p}
$$

where the measurement matrix $A$ is created from a set of selected rows from the DFT matrix. Regular CS approaches can be used to solve (2.23). However, assuming constant target range and acceleration for several PRI in a row as in [36] allows using the multiple measurement vectors (MMV) method for more robust signal recovery.

A hardware prototype for a sub-Nyquist radar was introduced in [38]. The implementation was achieved by using four parallel channels. A band pass filter is used in each channel to filter the signal spectrum to a distinct band. Afterwards, the output is demodulated to the baseband and sampled at its Nyquist rate. Finally, the outcome of four channels represents four sets of consecutive Fourier coefficients. 


\subsection{Conclusion}

In this chapter, we have introduced a brief description of the radar function and radar subsystems. The CS theory has been introduced in more details. The effect of $\mathrm{CS}$ on the detection performance and radar detectors functions is discussed showing promising results for the CS based radar. Moreover, we discuss two CS sampling process techniques, RD and MWC. Both techniques demonstrate the validity of CS approach in radar applications from the implementation point of view. 


\section{Chapter 3}

\section{Compressive Sensing in Pulse Radar using Prior Information}

In this chapter we discuss the pulse radar signal recovery problem. Our study is focused on how can we exploit the radar signal nature to help the reconstruction algorithm improve signal estimation and, consequently, improve the detection performance.

It is widely observed that prior information of the signal of interest might be useful for further improvements in the compressive sensing process. Thanks to a high PRF compared to the target speed, the difference between two successive PRIs is small. This special property of pulse radar signals can be exploited in the context of prior information. Hence, we introduce a new formula to deal with the prior information in pulsed radar along with an idea of exploiting the previously received signal to be used as prior information for the current signal in the new proposed reconstruction optimization formula. The performance of the compressive sensing reconstruction and detection are analyzed in different scene scenarios by means of ROC curves.

\subsection{Prior Information and Compressive Sensing}

The signal nature in CS, such as signal sparsity and SNR, affects the performance of the sensing process and the signal reconstruction process. Moreover, it is widely observed that prior knowledge about the signal can help in further reducing the sampling rate [39-41]. The prior information can be a signal that is similar to the signal of interest. As an example, consecutive video frames, most probably, have the same background. The only difference is the moving characters in the scene. 
A practical example is shown in [39] where a cardiac dynamic magnetic resonance imaging (MRI) image shows the heart moves between different frames. However, many surrounding structures remain essentially static. In the radar framework, the scene is mostly static. Moving targets only represent the variation between different frames.

As mentioned in Sec. 2.2, if we have an $M$-dimensional vector $y$, and a known measurement matrix $\Phi$, then $\ell_{1}$ minimization can be used to reconstruct the original signal $s$ as in (2.15). Assume that we have a sparse representation of an already reconstructed signal $w \in \mathbb{R}^{n}$, but similar to the original signal $s$. Suppose $g: \mathbb{R}^{n} \longrightarrow \mathbb{R}$ is a function that measures the similarity between $s$ and the prior information $w$, where $g(s-w)$ is chosen to be as small as possible. In this scenario, we can solve (2.15) but in a modified version as [42]

$$
\begin{gathered}
\min _{s}\|s\|_{1}+\beta g(s-w) \\
\text { s.t } \quad y=\Phi \Psi s
\end{gathered}
$$

where $\beta>0$ achieves the trade-off between signal sparsity and closeness (fidelity) to the prior information. In [42], two models are introduced to solve (3.1) given as

$$
\begin{gathered}
\min _{s}\|s\|_{1}+\beta\|s-w\|_{1} \\
\text { s.t } \quad y=\Phi \Psi s
\end{gathered}
$$

and

$$
\begin{gathered}
\min _{s}\|s\|_{1}+\beta\|s-w\|_{2}^{2} \\
\text { s.t } \quad y=\Phi \Psi s .
\end{gathered}
$$

The analysis and the experimental results in [42] found that (3.2) is much better than (3.3) for solving (3.1) with fewer number of measurements. Thus in this work, (3.2) will be considered in the analysis.

In [43], this approach was used with CS video background subtraction. The results achieve a dramatic reduction in the number of measurements compared to the classical 
compressive background subtraction schemes.

\subsection{Compressive Sensing in Radar Applications Using Prior Information}

We will consider the same concept of the discussed approach [42]. However, since we are interested in pulse radar signal, we will adapt some parameters and add some modifications to this approach to make it compatible with the nature of pulse radar signals.

\subsubsection{Motivations}

Before going deeply into our approach, we wish to highlight some points.

1. Sparsity of pulse radar signal. Every PRI is divided equally into a number of range cells. Assume that the width of the range cell is equal to the width of the transmitted pulse $\delta$. Hence, every single PRI contains $T / \delta$ range cells. Usually $\delta$ is very narrow (small) compared to a complete PRI $T$. i.e., $\delta \ll T$. The number of non-zero entries in one PRI represents the number of the detected targets in this PRI. However, it is important to notice that this number does not represent the total number of targets in the scene. To understand that clearly, we have to address the radar antenna movement.

In a surveillance radar as an example, the radar antenna scans $360^{\circ}$ scene. Let the antenna's horizontal beam width be $\Theta_{A} Z=1^{\circ}$, then the number of detected targets in a single PRI represents the total number of targets in a single angle $\theta$, which in our assumption is equal to $1 / 360$ of the scene.

Hence, the expected number of targets in a single PRI, in regular cases, is much less than the number of range cells (i.e., few non-zero entries). Hence, we can consider every single PRI as compressible sparse signal.

2. Similarity of the information between successive PRIs. We claim that the information of each PRI is similar to the next PRI, unless a target disappears or a new target appears. The received echoes from the same target within two consecutive periods come almost at the same time delay, especially for high $P R F$. To verify this claim, let us define the dwell time $t_{d w}$ as the time in seconds that 
an antenna beam spends on a target while scanning. Let the antenna scanning rate be $v_{a}$ revolution/ min. Let the number of hits that a target receives during the dwell time be denoted as $H$, which is equal to the number of PRIs during dwell time. Then the the dwell time can be defined as

$$
t_{d w}=\frac{\Theta .60}{360 v_{a}}=H T
$$

The range associated to a single range cell controls the radar range resolution which can be defined as

$$
R_{c}=\frac{c \delta}{2}
$$

where $c$ is the speed of light and $\delta$ is the transmitted pulse width.

The target relative speed to the radar during dwell time $v_{t g t}$ is defined as

$$
v_{r}=v_{t g t} \cos \alpha=\frac{d_{d w}}{t_{d w}}
$$

where $v_{t g t}$ is the real target speed and $\alpha$ represents the angle between direction of the reflected signal and the target flight direction. $d_{d w}$ represents the traveled distance by a target during dwell time. Then

$$
d_{d w}=v_{t g t} \cos \alpha t_{d w} .
$$

To verify our claim, we need to verify that the traveled distance by the target during the dwell time is less than the distance associated to a single range cell, i.e.,

$$
d_{d w} \leq R_{c}
$$

from (3.5) and (3.7), (3.8) can be written as

$$
\begin{aligned}
v_{t g t} \cos \alpha t_{d w} & \leq \frac{c \delta}{2} \\
v_{t g t} & \leq \frac{c \delta}{2} \frac{1}{\cos \alpha t_{d w}}
\end{aligned}
$$

From (3.4), 


$$
\begin{aligned}
v_{t g t} \leq & \frac{c \delta}{2} \frac{1}{\cos \alpha T H} \\
& \text { or } \\
v_{t g t} \leq & \frac{c \delta}{2} \frac{f_{r}}{\cos \alpha H} .
\end{aligned}
$$

where $f_{r}$ is the Doppler frequency.

Hence, (3.10) shows the upper bound for target speed to not migrate between range cells within $H$ PRIs. In our case, we consider two PRIs, $H=2$. Let us see a numerical example to get the sense of the target speed limits. Let us consider a radar with the following parameters. $T=0.4 \mathrm{~ms}, \delta=20 \mathrm{~ns}$. Let a target travel with an angle $\alpha=60^{\circ}$. From (3.10), the target delay is constant when the target speed does not exceed $7500 \mathrm{~km} / \mathrm{h}$ (about six times the speed of sound). Hence our claim is not only valid for targets with low speed, but also with reasonably high speed targets.

Hence, the probability that the target migrates from one range cell to another within two successive PRIs is low. Then, we can consider every PRI as prior information for the next one.

\subsubsection{Proposed Formula}

Since we have prior information for the signal of interest, we wish to exploit the prior information to improve the results of compressive sensing with radar signals. Let $s$ be the received signal within PRI $n$ and $w$ be the received signal from the previous PRI $n-1$. As discussed in Sec. 3.2.1, the pulse radar signal can be considered as a sparse signal directly in time domain, then $\Psi$ can be removed or set to be an identity matrix. We can formulate the convex problem as

$$
\begin{gathered}
\min _{s}\|s\|_{1}+\beta\left\|a^{T} \odot s-\tilde{w}\right\|_{1} \\
\text { s.t } \quad y=\Phi s
\end{gathered}
$$

where

$$
\tilde{w}_{i}= \begin{cases}w_{i}, & i \in V \\ 0, & \text { otherwise }\end{cases}
$$


and

$$
a_{i}= \begin{cases}1, & i \in V \\ \alpha, & \text { otherwise }\end{cases}
$$

where $\tilde{w}_{i}$ is the element $i$ in $\tilde{w}$. $V$ is the set of time indices in the vicinity of the expected target delay and $\alpha$ is a parameter that controls the noise suppression where targets are less expected. The notation $\odot$ in (3.11) denotes element-wise product. The potential advantage of this formula is that portions of the return signal where we do not expect a target, based on prior information, are suppressed by the parameter $\alpha$ in the optimization process (i.e., the noise is almost suppressed). Hence, the SNR of the reconstructed signal can be improved. Also, a better performance of $P_{d}$ and $P_{f a}$ can be achieved using fewer measurements as shown in Sec. 3.3. However, we should take into consideration that a new target might appear in the scene where we do not expect a target. Choosing $\alpha$ would handle the trade-off between $P_{d}$ and $P_{f a}$ as will be clearly seen in the analysis in Sec. 3.3. Seeking for a practical performance close to optimal, the value of $\beta$ can be set to unity [43].

When the target is moving too fast, it might cause a considerable change in the echo delay from one PRI to the next. In this scenario, $s-w$ might be large. Thus, this approach might not have the desired result. However, if a good prediction existed for the target movement then segments of $\tilde{w}$ could be shifted accordingly to improve the result of $s-w$. In other words, (3.12) could potentially be adapted with

$$
\tilde{w}_{i}= \begin{cases}w_{i-d_{i}}, & i \in V \\ 0, & \text { otherwise }\end{cases}
$$

where $d$ is a vector contains a set of shift amounts for moving targets. This kind of information can be derived from the radar tracker or by using tracking techniques such as with a Kalman filter [44].

\subsection{Analysis and Results}

In this section, our goal is to analyze the signal reconstruction performance of the proposed formula by considering the previous PRI as prior information for the current one. In our analysis, we consider the case of a pulse radar signal with a PRI, $T=$ $3 \mu$ sec divided into 150 range cells which provide a pulse width of $20 \mathrm{nsec}$. We consider 
the scenario where two target echoes have been received at the same amplitude with delays 10 and 100 range cells, respectively. Assume that the received signal has been contaminated with additive white Gaussian noise with zero mean and unit variance. In this chapter we have a simple signal model (signal and noise only) to address the effect of prior information on compressive sensing. We are going to introduce a more complicated signal model with clutter added in Chapter 5.

To be able to evaluate the performance of the proposed formula in (3.11) using prior information, we carried out the simulation in three different scenarios. For each scenario, 1000 Monte Carlo simulations are executed. The optimization process was carried out using CVX, a package for specifying and solving convex programs [45].

The measurement matrix $\Phi$ is chosen to be a normally distributed random matrix. A level of $50 \%$ of the target amplitude is used as a fixed detection threshold. The detection probability $P_{d}$ is estimated depending on detecting both targets and not only one. In other words, if both targets are detected, it counts as "Hit" and if no target or one target only detected, it counts as "Miss". $P_{f a}$ is computed over all output range cells except the target range cells with $50 \%$ target pulse width margin (i.e., any signal exceeds the fixed threshold along the reception period, except within the target vicinity, is considered as a false alarm).

To demonstrate the performance of the proposed formula, the results of the proposed approach are compared to the $\ell_{1}$ minimization approach without using any prior information as mentioned in (2.15), in our context, it will be denoted as the regular reconstruction or classical approach. The results are also compared to the reconstruction approach mentioned in (3.2) using prior information [42].

In the first scenario, we consider the case of two targets in the scene and evaluate the effect of the closeness of the prior information. The term closeness in our context means how much the target delays in the received signals match. So, a closeness of $0 \%$ is assigned for the two completely different signals. In other words, the target delay in the present signal $s$ lies outside of the target vicinity $V$ that is calculated based on the prior information. $100 \%$ is assigned for completely matched signals, i.e., $s-w=0$ in the vicinity of the expected targets.

In this scenario the $\mathrm{SNR}$ is set to $0 \mathrm{~dB} . P_{d}$ and $P_{f a}$ are measured for closeness percentage varying from $0 \%$ to $100 \%$ using the regular reconstruction by $\ell_{1}$ minimization and the proposed prior information approach with different values of $\alpha$. They are also compared to the prior information approach introduced in (3.2). Figure 3.1 
shows that $P_{d}$ is better when using the proposed formula setting $\alpha=0$. It is also shown that using the proposed formula introduces a noteworthy improvement in $P_{d}$ compared to the classical approach even if the closeness of the prior information is as low as $10 \%$.

On the other hand, Fig. 3.2 shows that decreasing $\alpha$ worsens $P_{f a}$ for the closeness less than 50\%. However, there is still a considerable improvement in $P_{f a}$ for the closeness greater than 50\%. It is clear from Fig. 3.1 and 3.2 that there is a trade-off between $P_{d}$ and $P_{f a}$ for different $\alpha$ values.

In the second scenario, we consider the case of two targets in the scene and address the effect of different SNR values on $P_{d}$ and $P_{f a}$ and the improvement of the reconstructed signal SNR. The simulation carried out within the SNR range from 2 $\mathrm{dB}$ to $8 \mathrm{~dB}$ and the closeness of the prior information is $40 \%$. Figure 3.3 shows the relation between $P_{d}$ and SNR. The ROC curve in Fig. 3.4 demonstrates the $P_{d}$ performance with different $P_{f a}$ at $\mathrm{SNR}=0 \mathrm{~dB}$. Both figures show that the $P_{d}$ performance is better than the classical approach over all SNR values. Figure 3.5 shows that the reconstructed signal SNR has an improvement by using the proposed approach over the classical reconstruction approach. It is noticed that decreasing $\alpha$ produces better reconstruction SNR.

In the third scenario, we consider the situation of one original target in the prior information $w$ while a new target appears in the current PRI $s$. This scenario simulates a regular case that takes place when a new target penetrates the radar field of detection. Figure 3.6 shows $P_{d}$ performance for each target individually in both approaches, using the proposed prior information approach and the classical one, in different SNR values. It is clear that the performance of $P_{d}$ by using the prior information surpasses the performance of the classical approach.

We can conclude from the simulation results that the proposed approach of CS using the previously received PRI as prior information for the current period introduces an improvement in probability of target detection. 


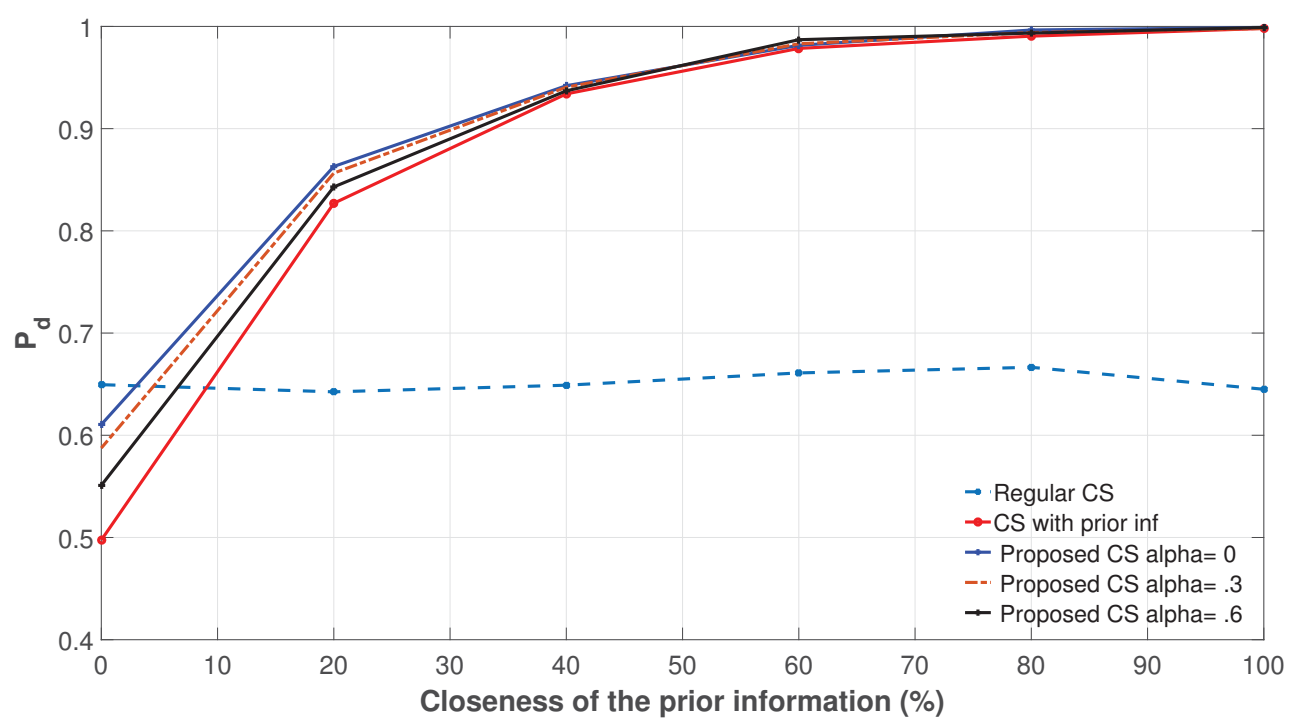

Figure 3.1: The relation between the closeness of the prior information and $P_{d}$.

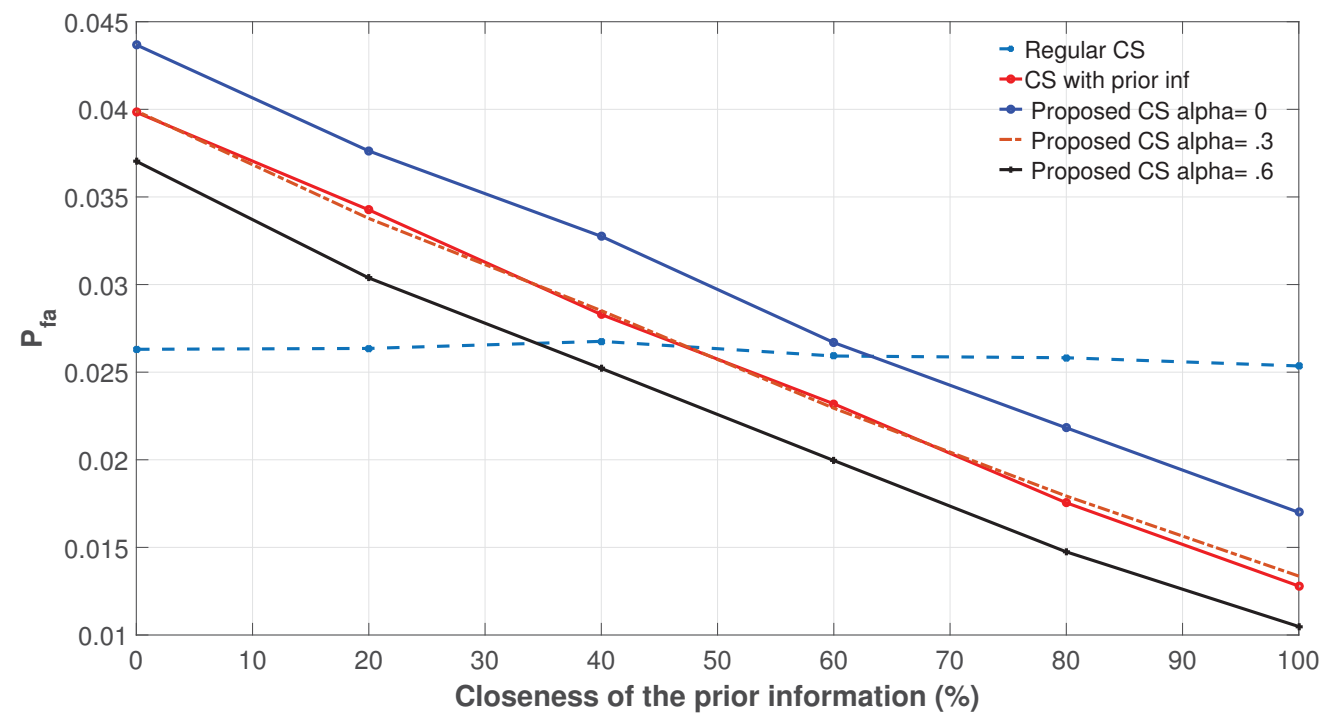

Figure 3.2: The relation between the closeness of the prior information and $P_{f a}$. 


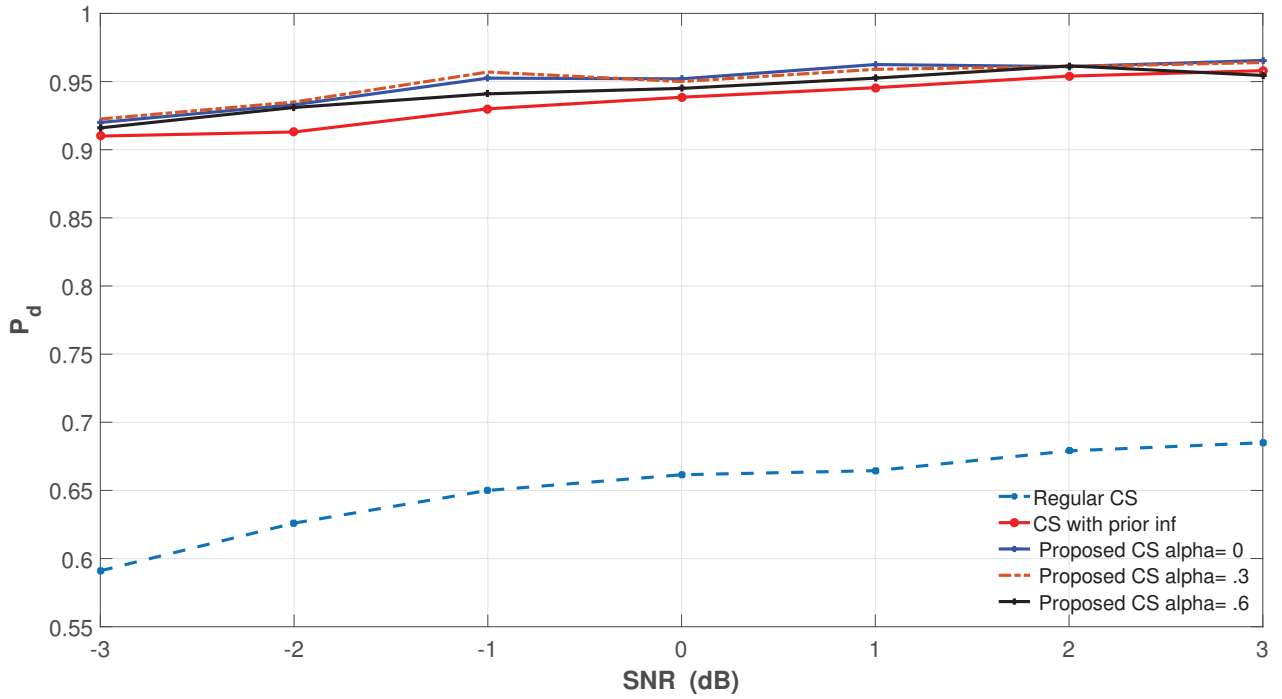

Figure 3.3: The relation between $\mathrm{SNR}$ and $P_{d}$.

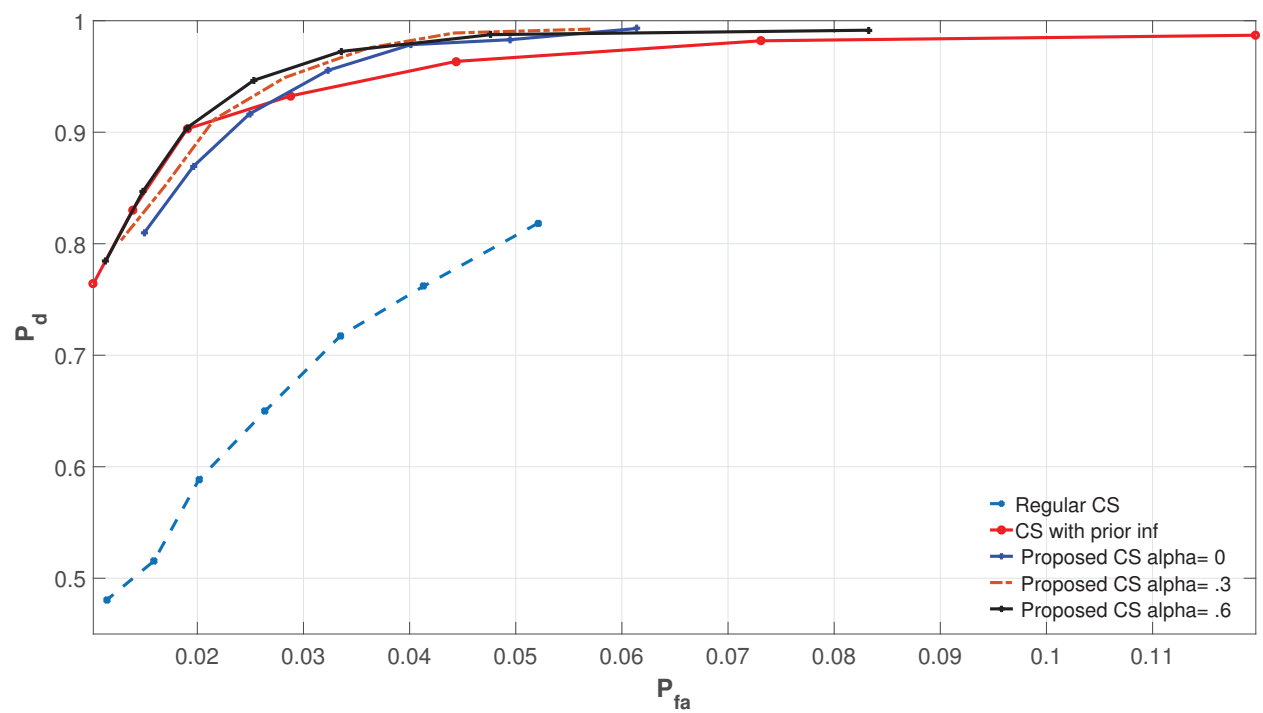

Figure 3.4: The relation between $P_{d}$ and $P_{f a}$. 


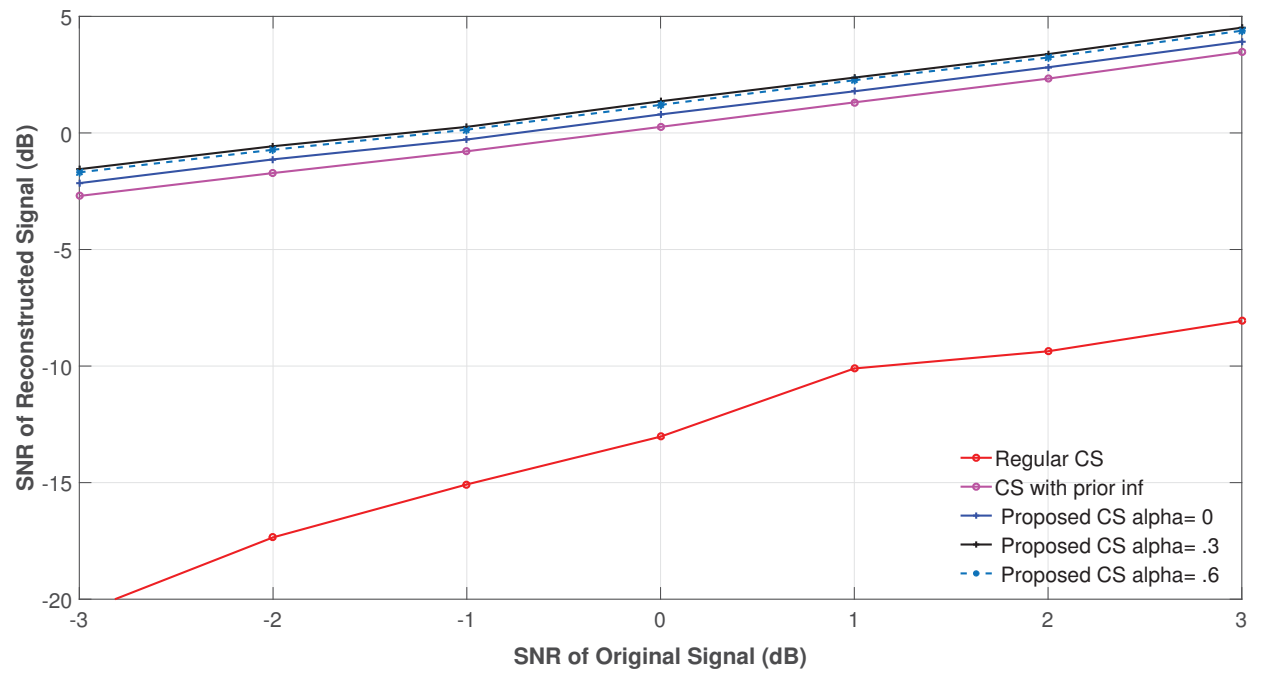

Figure 3.5: The relation between SNR of the original signal and SNR of the reconstructed signal.

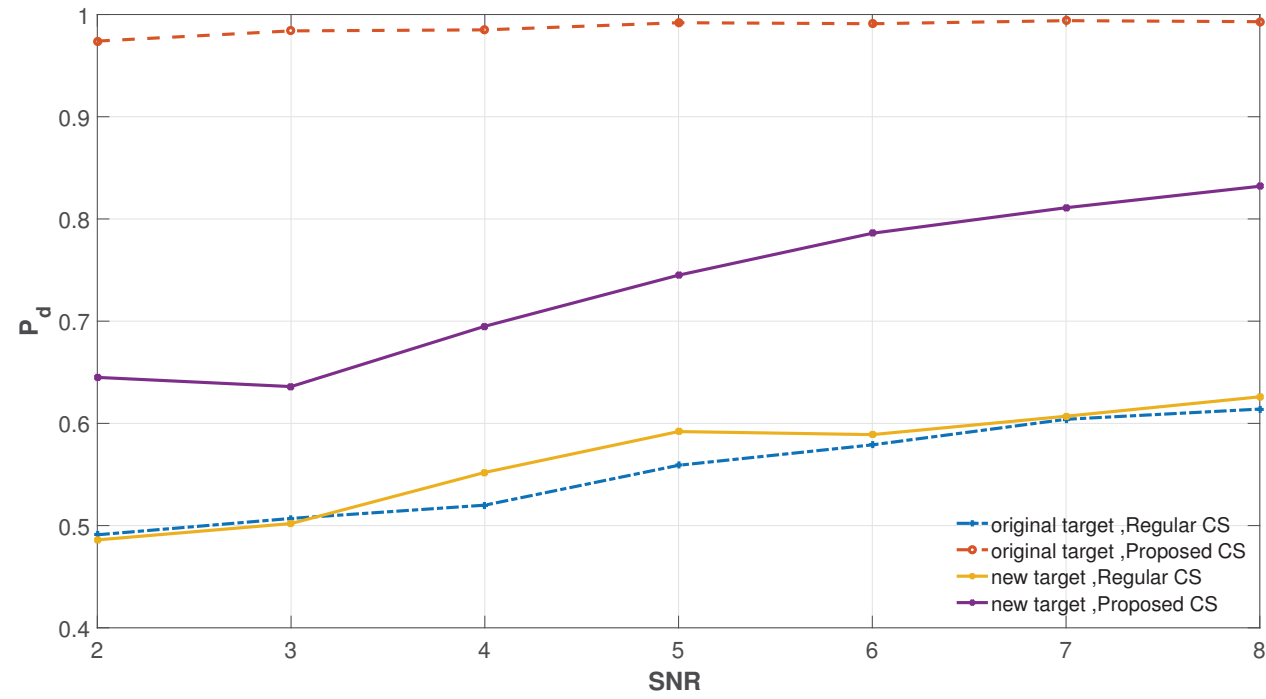

Figure 3.6: The relation between the SNR and $P_{d}$ for the original and the new target. 


\subsection{Conclusion}

In this chapter, we propose a formula to improve the utilization of the prior information in CS for the pulse radar signals and suggest using the previously received PRI as prior information for the current PRI. To study the feasibility of this proposition, the detection performance was investigated in different scenarios using ROC curves. The simulation results demonstrate that the performance of $P_{d}, P_{f a}$ and SNR are improved by using the proposed formula. The proposed formula and exploiting the previously received signal as prior information introduce improvements in the performance of the CS reconstruction compared to the classical approach. 


\section{Chapter 4}

\section{Adaptive Sub-Nyquist Sampling and Compressive Detection for Pulsed Radar}

In this chapter we focus on the problem of compressive sensing sampling process for a pulse radar signal corrupted by additive white Gaussian noise. We present a proposed algorithm using the simple Haar wavelet bases to adaptively sample the signal at a sub-Nyquist rate based on compressive sensing. The proposed algorithm uses the previously received pulse interval as prior information for the present interval. The detection performance of the proposed algorithm is evaluated using ROC curves for different values of SNR. Moreover, the reconstruction complexity is also evaluated using reconstruction time as a measure. The evaluation is carried out using different number of samples and compared to competing approaches. Using the Haar wavelet bases provides simple system design and implementation and also faster reconstruction.

\subsection{Adaptive Sub-Nyquist Sampling}

The main advantage of choosing the Fourier bases in the Xampling approach discussed in Sec. 2.5, is that it is easy to implement [36]. However, by using Fourier bases, we do not have any time localization. In other words, any Fourier coefficient spans the entire signal without exception. If we need to focus on a particular segment of the signal, we cannot do that using Fourier bases. This is where wavelet bases come into play. The multi-resolution analysis wavelet transform is localized in both time 
and frequency. Thus, in some radar applications, the wavelet transform is a valuable approach. We can find that wavelets are used in target detection [46], constant false alarm rate (CFAR) detectors [47], and clutter suppression and signal denoising [48].

The question now is why one would need the time localization? The time localization criterion allows us to divide the signal evenly into segments of a specified period of time. Then, we can classify these segments according to their priority and provide more focus on high priority segments. That should allow for important information (information of interest) extraction. Moreover, if we design the measurement matrix using multi-resolution analysis time localized basis functions, that would help the solver in the recovery process to reach to the optimum faster since all entries are multiplied by zero except at a few entries where the basis functions are localized.

As mentioned previously, our interest is the pulse radar signal. As we have discussed in Sec. 3.2.1, due to high PRF, the received signal in consecutive time periods is similar with high probability. We can exploit this advantage by considering the previous PRIs as prior information to the present PRI. Hence, we have prior information for the target echo time delay which is translated to the target range.

In this section, we propose an algorithm exploits that the prior information to deal with radar signal acquisition using Haar wavelet basis functions in order to provide lower latency than the Xampling approach that uses Fourier basis functions.

\subsubsection{Wavelets and Signal Model}

The signal can be represented by the wavelet bases in terms of translation (shift) and dilation versions of the scaling function $\Phi(t)$ and the wavelet function $\Psi(t)$. To keep the simplicity and ease of the implementation, we will consider the simplest wavelet, the Haar wavelet. The Haar scaling function, which is used to capture information at certain levels of detail, can be defined on [0,1) as [49]

$$
\Phi(t)=1, \quad t \in[0,1)
$$

and the Haar wavelet function is used to capture additional information from one subspace to the next and can be defined as

$$
\Psi(t)=\left\{\begin{aligned}
1, & \text { for } t \in[0,1 / 2) \\
-1, & \text { for } t \in[1 / 2,1)
\end{aligned}\right.
$$


For integers $j$ and $k$, the wavelet $\Psi_{j, k}(t)$ is the wavelet function dilated by a factor $2^{j}$ and translated to the location $2^{-j} k$ and can be represented as

$$
\Psi_{j, k}(t)=2^{j / 2} \Psi\left(2^{j} t-k\right)
$$

Then, any given signal can be represented as

$$
x(t)=\sum_{k=-\infty}^{\infty} c_{k} \Phi_{0, k}(t)+\sum_{j=0}^{\infty} \sum_{k=-\infty}^{\infty} d_{j, k} \Psi_{j, k}(t)
$$

where $c_{k}$ is a constant and $\Phi_{0, k}(t)=\Phi(t-k)$. The wavelet coefficient $d_{j, k}$ can be found by

$$
d_{j, k}=\int_{-\infty}^{\infty} x(t) \Psi_{j, k}(t) d t
$$

By increasing the scaling factor $j$, one can zoom into a deeper signal detail. Thus, the wavelet transform acts as a mathematical microscope.

Let $x(t)$ represents the received signal for one PRI. Then

$$
x(t)=\sum_{p=1}^{N} a_{p} h(t-p)+n
$$

where $a_{p}$ is the scatter coefficient of the target echo at time delay $p$. The scatter coefficient value depends on some factors. Mainly, it depends on the radar target cross-section and some propagation factors. $h(t)$ is the transmitted pulse, $N=T / \delta$ is the number of range cells and $n$ is white Gaussian noise.

Since $\delta \ll T, N$ is very large. The expected number of echoes at one PRI represents the number of detected targets at certain angle in space within the radar detection range. Hence, the expected number of nonzero entries in one PRI is much less than the number of range cells $N$. Hence, the received signal is considered sparse in the time domain.

However, the receiver noise $n$ will affect the sparsity level. Thus, we should improve the SNR to an acceptable level for the signal recovery. In order to improve the SNR, usually a pulse integrator is applied by coherent integration of $I$ sweeps. In our case, we can apply the same technique by integrating the compressed signal for $I$ successive pulse intervals. Choosing $I$ depends on the signal SNR value and the required improvement factor. 
We consider a coherent pulse interval (CPI) that involves $I$ PRIs. During one CPI, we make the following assumptions.

1. Constant scatter coefficient for each target. In other words, we assume that the target fluctuations do not affect the target radar cross-section.

2. Constant pulse repetition frequency $(\mathrm{PRF})$.

3. Constant target delay. That means that the target speed does not allow the echo to migrate to the next range cell during single CPI. This assumption is reasonable and was verified in Sec. 3.2.1.

4. Single target in a single range bin.

5. The received signal is contaminated by a white Gaussian noise with zero mean and variance $\sigma^{2}$.

6. Number of targets is much less than number of range cells.

We can define our CS problem as

$$
y=W_{H} x
$$

where $W_{H}$ is a $M \times N$ matrix composed of selected $M$ Haar wavelet basis functions.

\subsubsection{Algorithm Motivations}

Applying the Xampling approach to radar signals allows the signal parameters to be acquired. However, in the proposed algorithm, we consider using the wavelet bases instead of Fourier bases as demonstrated in Fig. 4.2. We introduce an algorithm that exploits the features of Xampling approach and wavelet multi-resolution analysis to apply an adaptive sampling to the radar signal at a sub-Nyquist rate in much lower processing time compared to the Fourier approach in [36]. The proposed algorithm also exploits the previously received signals as prior information.

The main merits of our proposed algorithm are as follows:

1. The ability to choose a suitable bases - using the proposed algorithm allows us to select the required wavelet bases to be used in the signal sampling process among the complete multi-resolution wavelet bases. The bases are chosen to provide a nonuniform sampling structure to suit the signal nature. 
2. Time localization- Since the proposed algorithm uses wavelet bases, that allows for time localization. This feature can be exploited to choose the bases such that more focus can be applied to the time segments where a target is expected.

3. Adaptability - The proposed algorithm adaptively changes the number and the index of the selected bases to achieve a higher probability of detection.

4. Simplicity - the Haar wavelet bases are chosen in the proposed algorithm to make the implementation simple since it contains only real values of \pm 1 , which is much simpler compared to complex Fourier bases.

\subsubsection{Algorithm Procedures}

The proposed Algorithm 1 provides simple procedures to acquire the radar signal using Xampling approach and Haar wavelet bases. The signal can be divided into $T / 2 \epsilon$ time segments each of width $2 \epsilon$. The value of $\epsilon$ can be chosen to be several multiples of the pulse width $\delta$. The segments are classified into low priority segments where no target is expected and high priority segments where a target is expected (denoted by "target potential").

The algorithm provides a sample selection criteria such that the entire signal is sampled in a low resolution (i.e., lower number of samples) except for the target potential segments that are sampled at a higher resolution (i.e., higher number of samples). In case that an echo is received in any low priority segment, it can be detected as a target potential and considered in the next pulse interval with high priority by choosing more coefficients, which means more samples, at the new echo delay.

We apply Xampling to sample the pulse interval $n$ using a subset of Haar wavelet coefficients in lower levels, $\Psi_{j_{c}, k_{c}}(t)$ (i.e., coarse resolution). The compressed signal is integrated for $I$ PRIs to improve the SNR as follows.

$$
\begin{gathered}
d_{j, k}=\int_{0}^{T} x(t) \Psi_{j_{c}, k_{c}}(t) d t . \\
\left(d_{j, k}\right)_{I}=\frac{1}{I} \sum_{\tilde{i}=1}^{I}\left(d_{j, k}\right)_{\tilde{i}} .
\end{gathered}
$$


Then, the signal is reconstructed using the CS recovery approach

$$
\tilde{x}_{p}=\underset{x_{p}}{\operatorname{argmin}}\left\{\left\|x_{p}\right\|_{1}: y=A x_{p}\right\} .
$$

Afterwards, the target potential detection takes place by applying an adaptive threshold using the CFAR approach after each signal recovery

$$
\tilde{x}_{p} \underset{H_{0}}{\stackrel{H_{1}}{\gtrless}} \gamma[t]
$$

where $H_{1}$ and $H_{0}$ represent the hypothesis of target presence and absence, respectively. $\gamma$ is the adaptive threshold created by the CFAR algorithm.

In this step, the CFAR detector is used only for estimating the target potential delays (i.e., high priority segments), not for the target detection. Hence, to discriminate between the detection results in this step and the next one, we denote the target that exceeds the CFAR threshold by target potential detection and the noise that exceeds the threshold by false target potential. The probability of false target potential $\left(P_{f t p}\right)$ is fixed to achieve high probability of target potential detection $\left(P_{t p d}\right)$. The $P_{f t p}$ value will introduce a trade-off between $P_{t p d}$ and the number of samples, where each target potential in pulse interval $n$ will introduce an increment in the number of samples to be chosen in the interval $n+1$.

Based on the CFAR threshold $(\gamma)$, the segment's priority is classified into high priority, where a potential of a target is detected, and low priority for the rest of the segments. The width of the high priority segment is controlled by $\epsilon$ which is a design parameter. However, increasing $\epsilon$ will increase the number of samples. Thus, it should be chosen carefully. The coefficients of higher levels, $\Psi_{j_{f}, k_{f}}(t)$, are selected (i.e., fine resolution). Then, the higher and lower level coefficients are merged to be used in the next PRI

$$
\Psi_{j, k}(t)=\Psi_{j_{c}, k_{c}}(t) \cup \Psi_{j_{f}, k_{f}}(t)
$$

Figure 4.3 shows a demonstration of the wavelet bases selection at coarse and fine resolutions. It shows the window around the target potential segment and demonstrates how the higher level bases are chosen to provide a higher signal resolution 


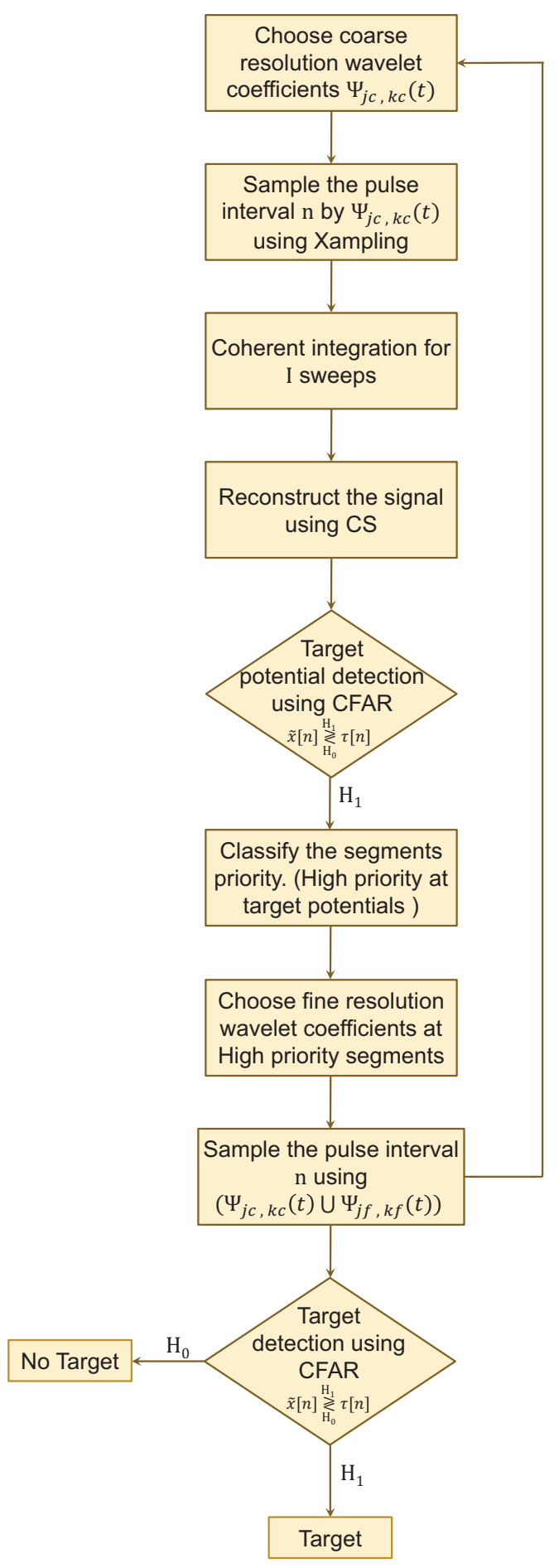

Figure 4.1: Flowchart illustrates the procedures of the proposed adaptive subNyquit rate algorithm using Haar wavelet coefficients. 


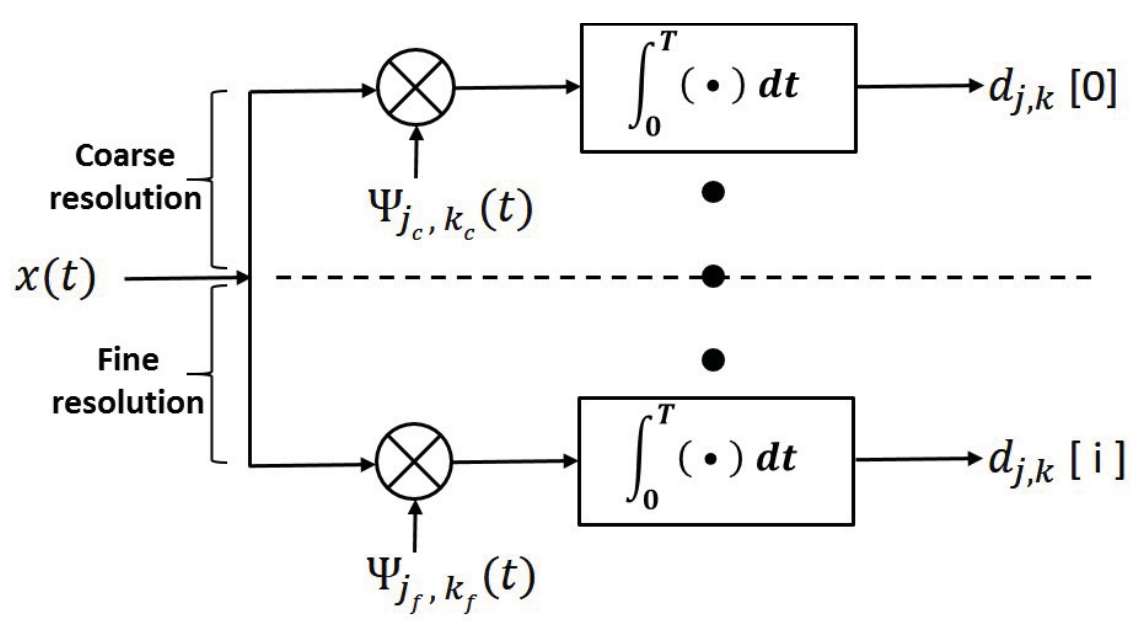

Figure 4.2: Sampling using wavelet basis functions.

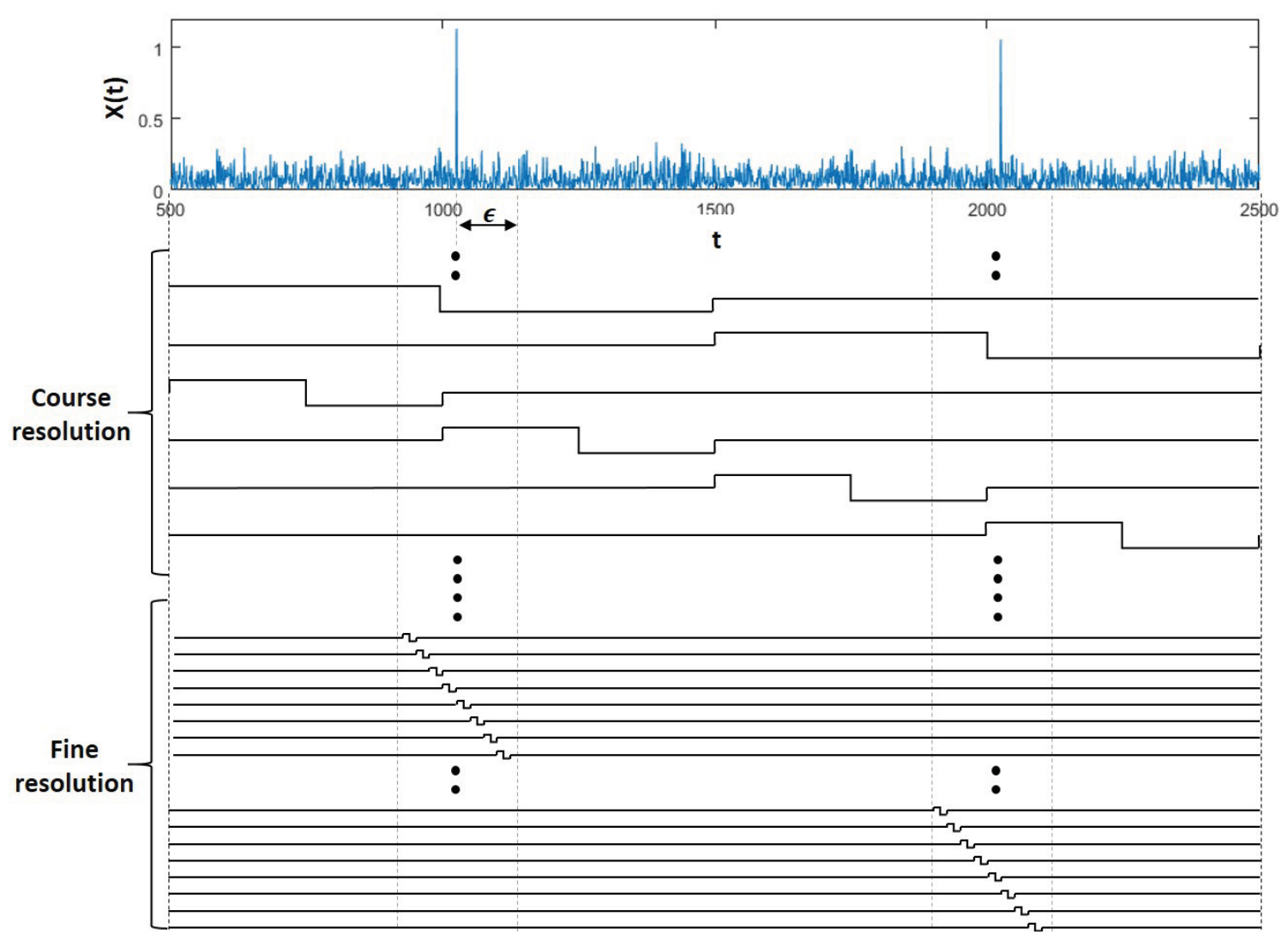

Figure 4.3: Demonstration of the Haar wavelet coefficients selection. The coarse resolution bases are selected along the signal. The fine resolution bases are selected only within a window where there is target potential. 


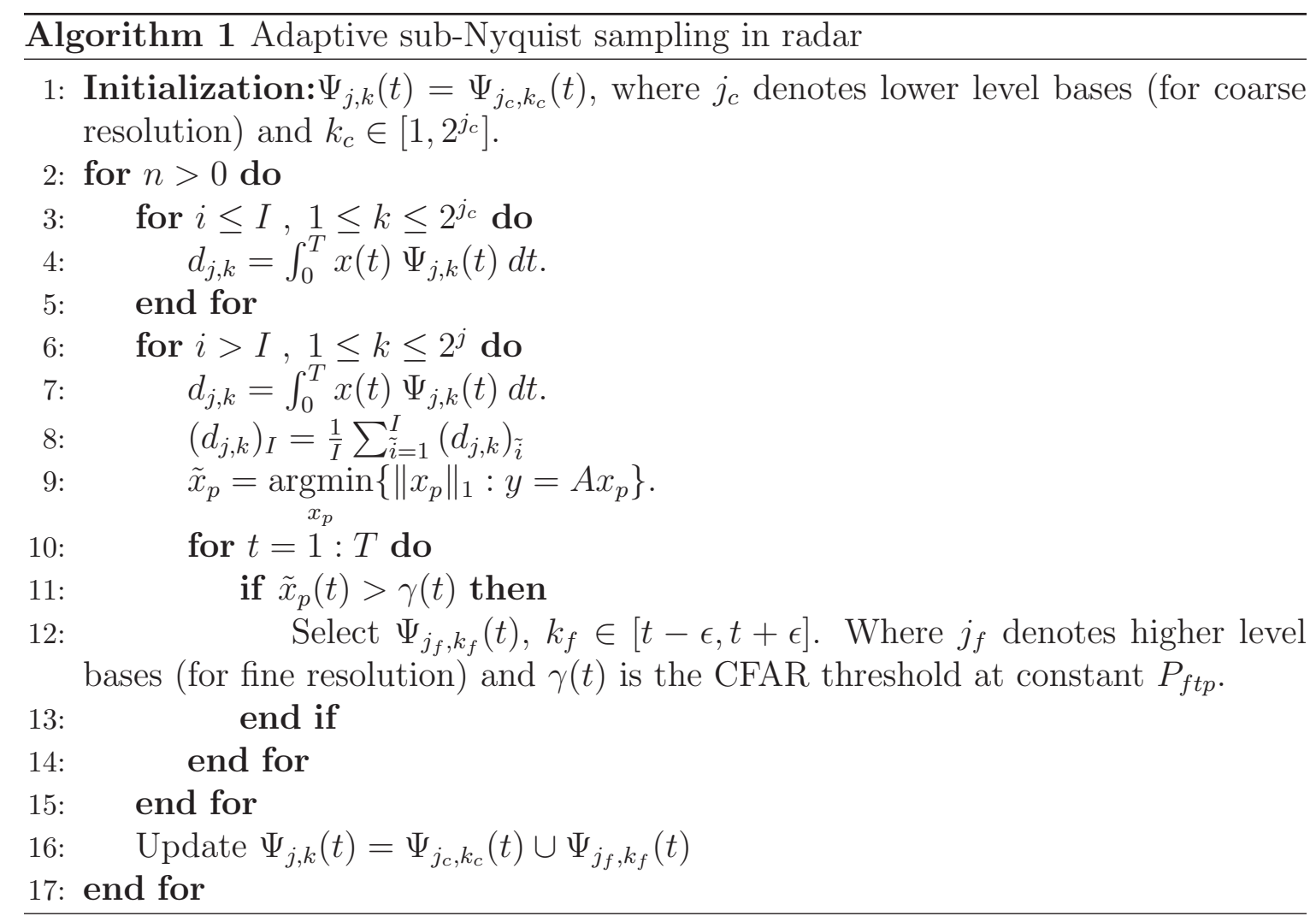

within this segment. The flowchart in Fig. 4.1 describes Algorithm 1 procedures.

\subsection{Analysis and Results}

In this section, we introduce the performance analysis of the proposed algorithm. To evaluate the algorithm performance, we compare the proposed algorithm with Xampling Fourier coefficients approach [4,35], mentioned in Sec. 4.1. In order to introduce an impartial study, we should address the performance of both approaches in terms of the signal recovery processing time, the probability of target detection $\left(P_{d}\right)$, and the relation between the number of required samples and reconstruction performance.

In our analysis, we consider the case of a pulsed radar signal with a pulse interval $T=100 \mu$ sec and pulse width $\delta=50 \mathrm{nsec}$ corresponding to $10 \mathrm{MHz}$ bandwidth (single-sided band). The signal was generated via MATLAB using 4096 samples which correspond to more than twice the Nyquist rate samples (2000 samples). The signal is corrupted by additive white Gaussian noise with zero mean and variance $\sigma^{2}$. 


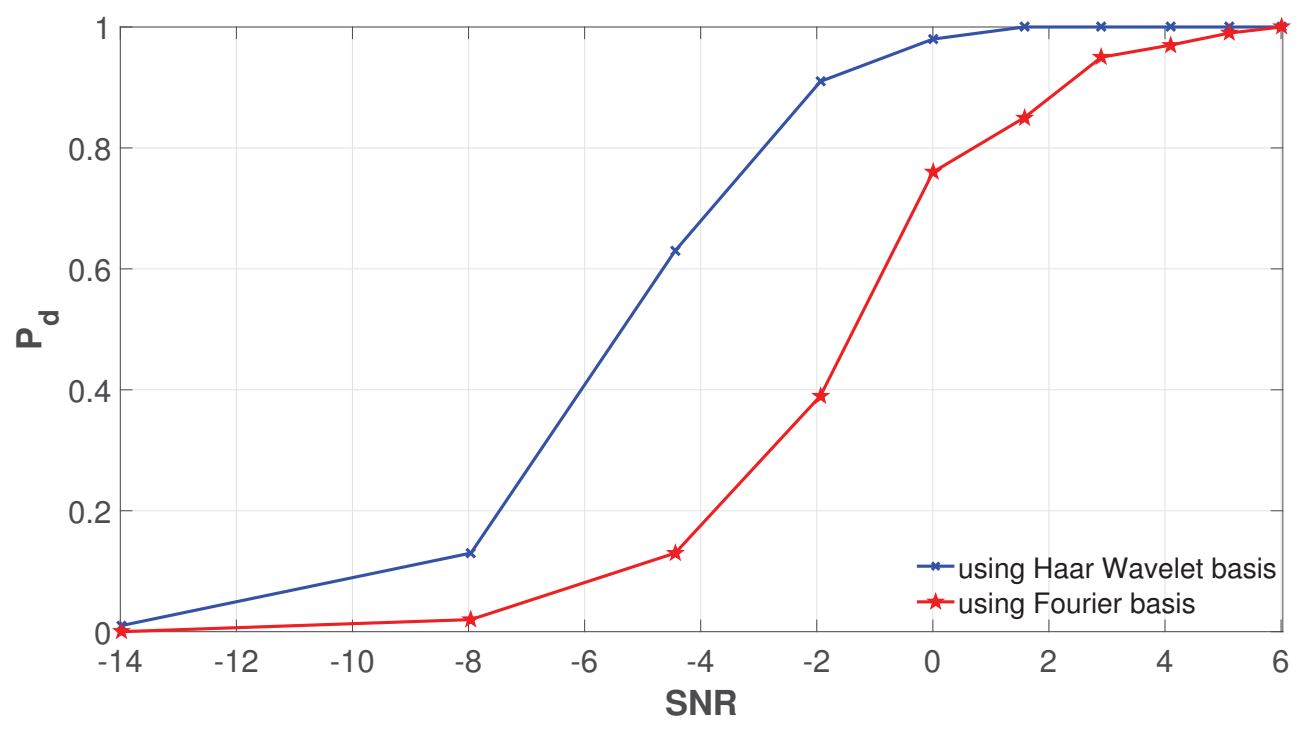

Figure 4.4: ROC curve using both Haar wavelet and Fourier bases $\left(P_{f a}=10^{-5}\right)$.

The optimization process in the signal recovery step was carried out using CVX, a package for specifying and solving convex programs [45].

To evaluate $P_{d}$, we used a CFAR detector at a constant probability of false alarm $\left(P_{f a}\right)$ of $10^{-5}$. During this experiment, the signal was recovered using 277 coefficients which correspond to $13.85 \%$ of the Nyquist rate samples for detecting one target in the scene. The selected wavelet coefficients include 256 fixed lower level coefficients (for coarse resolution), and the rest are the higher level coefficients (for fine resolution). The proposed algorithm uses an extra $8 \%$ of wavelet coefficients for each new target. The evaluation is carried out using the receiver operating characteristic (ROC) curves. The ROC curve is a measure of the receiver performance. It shows either a relation between $P_{d}$ and $P_{f a}$ for certain SNR value or a relation between SNR and $P_{d}$ for constant $P_{f a}$. Since we are using a CFAR detector, we use the latter one. In Fig. 4.4, ROC curve shows an improvement in $P_{d}$ using the proposed algorithm for different $S N R$ values. In order to obtain statistically stable results, the experiments were repeated 100 times and the average was taken for each value of the $S N R$. The $S N R$ was defined as

$$
S N R=\frac{|a|^{2}}{\sigma^{2}}
$$

where $a^{2}$ is the scatterer coefficient. However, since the proposed algorithm extracts the information from pulse interval $n$ and applies the result to pulse interval $n+1$, 
the target detection was carried out after signal recovery for the pulse interval $n+1$. In other words, the result of our proposed algorithm is delayed by one PRI ( $T$ sec) compared to the Fourier coefficients approach.

Since the number of required coefficients in the introduced algorithm is variable based on the number of target potentials detected in each pulse interval, it is hard to have an accurate comparison in terms of the required samples in both discussed approaches.

Moreover, regarding the Fourier coefficient approach, we do not have a coefficient selection criterion except that is mentioned in [38]. It is demonstrated in [38] that the coefficients should be a constellation consisting of consecutive coefficients selected from distributed frequency bands. On the other hand, the proposed approach not only provides the required number of coefficients but also provides specific indices of these coefficients.

We measured the effect of the number of selected coefficients on the signal from two perspectives: the reconstruction complexity and the reconstruction performance. Table 4.1 shows the results of the average recovery processing time and the average number of iterations for both approaches for different number of coefficients. The signal recovery processing time was estimated using the CVX parameter "cvx_ cputime". The results show that the proposed algorithm achieves a recovery processing time reduction compared to the Fourier coefficient approach, especially when the number of coefficients increases. For instance, the proposed algorithm is 93 times faster when 256 coefficients are selected and reaches 387 times faster when 512 coefficients are selected.

Moreover, Table 4.2 shows that the $P_{d}$ records an increase for the proposed algorithm for different number of coefficients. The results in Tables 4.1 and 4.2 have been calculated over an average of 300 runs at $S N R=-2 \mathrm{~dB}$.

\subsection{Conclusion}

In this chapter, we introduce a proposed algorithm for sub-Nyquist sampling of pulse radar signals based on the simple Haar wavelet coefficients. The signal recovery process has been carried out based on a CS approach. The proposed algorithm exploits the previously received pulse interval to use as prior information for the 
Table 4.1: Recovery statistics for different number of coefficients

\begin{tabular}{cccccc}
\hline & \multicolumn{2}{c}{ Recovery time (sec) } & & \multicolumn{2}{c}{ Average No. Iterations } \\
\cline { 2 - 3 } \cline { 5 - 6 } No. Coefficients & $\begin{array}{c}\text { Fourier } \\
\text { bases }\end{array}$ & $\begin{array}{c}\text { Haar wavelet } \\
\text { bases }\end{array}$ & & $\begin{array}{c}\text { Fourier } \\
\text { bases }\end{array}$ & $\begin{array}{c}\text { Haar wavelet } \\
\text { bases }\end{array}$ \\
\hline 32 & 1.9249 & 0.5514 & & 23.95 & 9.06 \\
64 & 3.5448 & 0.5478 & & 23.18 & 9.16 \\
128 & 10.0089 & 0.6446 & & 23.71 & 9.39 \\
256 & 63.9480 & 0.6885 & & 23.03 & 9.65 \\
277 & 73.3718 & 0.6742 & & 23.37 & 9.75 \\
512 & 239.6935 & 0.6193 & & 22.43 & 9.96 \\
\hline
\end{tabular}

Table 4.2: Reconstruction performance for different number of coefficients

\begin{tabular}{ccc}
\hline & \multicolumn{2}{c}{ Probability of detection $\left(P_{d}\right)$} \\
\cline { 2 - 3 } No. Coefficients & $\begin{array}{c}\text { Fourier } \\
\text { bases }\end{array}$ & $\begin{array}{c}\text { Haar wavelet } \\
\text { bases }\end{array}$ \\
\hline 32 & 0.02 & 0 \\
64 & 0.06 & 0 \\
128 & 0.18 & 0.45 \\
256 & 0.38 & 0.87 \\
277 & 0.39 & 0.91 \\
512 & 0.98 & 1.00 \\
\hline
\end{tabular}


present interval. The algorithm provides sampling at a high resolution where there is target potential and lower resolution otherwise. The proposed algorithm performance was evaluated and compared with the Fourier coefficients approach. The simulation results show that the proposed algorithm is up to 387 times faster than the Fourier coefficients approach and achieve an improvement in the target detection probability $P_{d}$ for all SNR values for different number of coefficients while the total number of samples are $13.85 \%$ of the Nyquist rate samples. 


\section{Chapter 5}

\section{Frequency and Phase Analysis Using S-transform}

In this chapter, we introduce a new algorithm that exploits the S-transform basis functions properties to allow extracting the target Doppler information. This chapter paves the way to build a low latency compressive sensing sub-Nyquist coherent pulse radar receiver capable of detecting the required target parameters which include the target range (represented by the pulse time delay) and the target speed (represented by its Doppler frequency), all without the computationally expensive signal reconstruction.

\subsection{Motivations}

The required target information depends on the radar application. Some radar applications require only the target range. Others require more information about the target such as target speed, target radar cross section (RCS) and target tracking.

As discussed in Sec. 2.1.1, the target velocity can be detected by estimating the Doppler frequency shift. However, the Doppler frequency shift would be small (on the order of a few $\mathrm{Hz}$ ) relative to the carrier frequency (on the order of many $\mathrm{MHz}$ ). Usually, a phase comparison is carried out to determine the Doppler frequency shift instead of direct frequency comparison. In such cases, a coherent radar system is usually used.

In coherent radar systems, a copy of the transmitted pulse is kept as a reference

for the carrier frequency and the phase information. Hence, the system has to keep high phase stability in the transmitted frequency. 
In order to extract target velocity information, the frequency and phase information is needed. Unfortunately, the Haar wavelet-based algorithm in Sec. 4.1 does not provide sufficient phase information. The phase information in Haar wavelet analysis is not clear. It does not have a direct relationship to the Fourier transform. Thus, to be able to extract the target Doppler information, we have to develop the algorithm by choosing other bases that provide clear phase information and have time localization.

\subsection{S-transform Background}

The S-transform, introduced in [50], is a time-frequency transformation. The key feature of the S-transform is that it not only provides frequency-dependent resolution but also combines it with absolutely referenced local phase information. The absolute reference phase means that $t=0$ is always used as a reference time for the phase information in the S-transform. The S-transform provides time localization and frequency localization and keeps the phase information as the phase of a component of the Fourier transform. Hence, the S-transform combines the advantages of wavelet multi-resolution analysis and the Fourier transform.

The direct relation between the S-transform and wavelets can be represented as a special mother wavelet multiplied by a phase factor [50]

$$
S(\tau, f)=e^{i 2 \pi f \tau} W(\tau, d)
$$

where $d$, the dilation factor, is equal to the inverse of the frequency $f$. $\tau$ represents the time shift. The mother wavelet in this case is represented as

$$
w(t, f)=\frac{|f|}{\sqrt{2 \pi}} e^{-\frac{t^{2} f^{2}}{2}} e^{-i 2 \pi f t}
$$

Hence, the S-transform of a function $h(t)$ is defined as [50]

$$
S(\tau, f)=\int_{-\infty}^{\infty} h(t) \frac{|f|}{\sqrt{2 \pi}} e^{-\frac{(\tau-t)^{2} f^{2}}{2}} e^{-i 2 \pi f t} d t .
$$

The unique property of the S-transform is that the Fourier spectrum can be derived simply by averaging the spectra over time as follows [50] 


$$
\int_{-\infty}^{\infty} S(\tau, f) d \tau=H(f)
$$

where $H(f)$ is the Fourier transform of $h(t)$. Hence, $h(t)$ can be recovered exactly from $S(\tau, f)$ as

$$
h(t)=\int_{-\infty}^{\infty}\left\{\int_{-\infty}^{\infty} S(\tau, f) d \tau\right\} e^{i 2 \pi f t} d f .
$$

For a constant frequency $f_{0}$, the S-transform $S\left(\tau, f_{0}\right)$ is called a voice [50]. The voice is a one dimensional function defined as

$$
S\left(\tau, f_{0}\right)=A\left(\tau, f_{0}\right) e^{i \Phi\left(\tau, f_{0}\right)}
$$

where $\Phi\left(\tau, f_{0}\right)$ is the absolute phase information for the isolated frequency component $f_{0}[50]$.

Similarly, for a signal $h(t)=A \exp \left(i 2 \pi f_{0} t+\phi\right)$, the Fourier transform at frequency $f_{0}$ will return the amplitude $A$ and the phase $\phi$. Hence, the S-transform provides the same amplitude and phase information as the Fourier transform in a fixed frequency. Thus, the S-transform can be considered as a generalization of the Fourier transform for non-stationary signals.

In the discrete case, S-transform basis functions can be represented as $N^{2}$ localized Fourier basis vectors. Each Fourier basis vector is divided into $N$ localized vectors by a Hadamard product with $\mathrm{N}$ shifted Gaussian windows. Hadamard product can be simply defined as an element-wise matrices product. Let us define $h[k T]$ as the discrete time series where $k=0,1, \ldots, N-1$. Then, the discrete S-transform can be represented as

$$
S(m, n)=\sum_{k=0}^{N-1} h[k T] \frac{|n|}{\sqrt{2 \pi N}} e^{-\frac{(m-k)^{2} n^{2}}{2 N^{2}}} e^{-j 2 \pi \frac{n k}{N}} .
$$

Hence, the discrete S-transform matrix can be represented as 


$$
D S T=\left[\begin{array}{cccc}
s_{1,1}(1) & s_{1,1}(2) & \cdots & s_{1,1}(N) \\
s_{1,2}(1) & s_{1,2}(2) & \cdots & s_{1,2}(N) \\
\vdots & \vdots & \cdots & \vdots \\
s_{1, N}(1) & s_{1, N}(2) & \cdots & s_{1, N}(N) \\
s_{2,1}(1) & s_{2,1}(2) & \cdots & s_{2,1}(N) \\
\vdots & \vdots & \cdots & \vdots \\
s_{N, N}(1) & s_{N, N}(2) & \cdots & s_{N, N}(N)
\end{array}\right]
$$

where

$$
s_{m, n}(k)=e^{-\frac{f^{2}(m-k)^{2}}{2 N^{2}}} e^{-j 2 \pi \frac{n k}{N}} .
$$

The S-transform represents redundancy in the time-frequency plane. Hence, an efficient representation of the S-transform, the discrete orthogonal S-transform (DOST) is introduced in [51]. DOST is a discrete orthogonal time-frequency version of the S-transform. For an N-point time series $h[k T]$

$$
S\{h[k T]\}=S\left(v T, \frac{v}{N T}\right)=\sum_{k=0}^{k=N-1} h[k T] S_{[v, \beta, \tau]}[k T]
$$

where $v$ is the frequency variable that indicates the center of the frequency band of a particular voice and $\beta$ indicates the frequency bandwidth of the voice. $\tau$ is the time variable that indicates the time localization (the voice time shift) and $S_{[v, \beta, \tau]}[k T]$ indicates the DOST basis functions which can be defined as [51]

$$
S_{[v, \beta, \tau]}[k T]=\frac{i e^{-i \pi \tau}}{\sqrt{\beta}} \frac{\left\{e^{-i 2 \pi(k / N-\tau / \beta)(v-\beta / 2-1 / 2)}-e^{-i 2 \pi(k / N-\tau / \beta)(v+\beta / 2-1 / 2)}\right\}}{2 \sin [\pi(k / N-\tau / \beta)]}
$$

To ensure orthogonality, $\tau \in[1, \beta-1]$ and values of $v$ and $\beta$ must be chosen such that each Fourier frequency is used only once [51]. However, in the case of compressive sensing, we should select a subset of these basis functions.

The localized cross spectral function (LCSF) of two time series $h_{1}[k T]$ and $h_{2}[k T]$ with $\operatorname{DOST} S_{1}(\tau, v)$ and $S_{2}(\tau, v)$ respectively is defined as [51] 


$$
\operatorname{LCSF}(\tau, v)=S_{1}(\tau, v) S_{2}^{*}(\tau, v)=A(\tau, v) e^{i \phi(\tau, v)}
$$

where $\left(^{*}\right)$ denotes complex conjugate. LCSF clearly identifies the cross spectral amplitude $A$ and the phase shift $\phi(\tau, v)$ for a given voice $v$ and time index $\tau$. Hence, it is a straightforward approach to measure the phase shift between 2 different signals instantaneously.

\subsection{Adaptive Sub-Nyquist S-transform Algorithm}

\subsubsection{Signal Model}

We consider a problem of a mono-static pulse radar. The transmitted signal is rectangular shape pulse with a duration $\delta$ and a constant frequency $F_{I F}$ during the pulse duration. Each target is defined by three parameters.

1. Time delay $\left(t_{d}\right)$, which is proportional to target range $R$ from the radar with the relation

$$
t_{d}=\frac{2 R}{c}
$$

2. Doppler Frequency $\left(f_{d}\right)$, which is proportional to target speed with the relation

$$
f_{d}=\frac{2 v_{r}}{\lambda}
$$

where $v_{r}$ is the target radial velocity and $\lambda$ is the wavelength.

3. Scatter coefficient $(a)$, which is related to radar target cross-section and propagation factors. It is also defines the target peak power.

We consider a coherent pulse interval (CPI) that involves I PRIs. During one CPI, we make the following assumptions.

1. Constant scatter coefficient for each target. In other words, we assume that the target fluctuations do not affect the target radar cross-section.

2. Constant pulse repetition frequency $(\mathrm{PRF})$.

3. Constant target delay. That means that the target speed does not allow the echo to migrate to the next range cell during single CPI. This assumption is reasonable even for fast moving targets as shown in Sec. 3.2.1. 
4. Constant target velocity. In other words, the target does not accelerate during the CPI. Accordingly, the target Doppler frequency $f_{d}$ is constant.

5. Single target in a single range bin. In other words, if the distance between two targets is less than the radar range resolution, they will be considered one target. The range resolution is defined as

$$
R_{r}=\frac{c \delta}{2}
$$

where $c$ is the speed of light and $\delta$ is the pulse width.

6. The expected Doppler frequency $f_{d}$ is within a previously known bandwidth $B_{f_{d}}$.

7. The received signal is contaminated by a white Gaussian noise with zero mean and variance $\sigma^{2}$.

8. The probability of receiving echoes from the scatterers are statistically independent. Since weather, rain, sea or land clutters are usually composed of many small scatterers, this assumption allows us to use Rayleigh distribution for clutter modelling [2].

9. Number of targets is much less than number of range cells.

Let $x(t)$ represents the received signal for one PRI. Then

$$
x(t)=\sum_{p=1}^{N} a_{p} h(t-p) e^{-j 2 \pi f_{d} t}+n(t)
$$

where $h(t)$ is the transmitted pulse and $N=T / \delta$ is the number of range cells. $n(t)$ is additive white Gaussian noise.

The scatter coefficients vector $\left\{a_{i}: i \in\{1, \ldots N\}\right\}$ has values at echo delays only and zero otherwise. Since we assume that the number of targets is much less than the number of range cells, then

$$
\|a\|_{0}:=\sum_{i=1}^{N} 1_{\left\{a_{i} \neq 0\right\}}(x)=k \ll N .
$$


Hence, $a_{i}$ is considered sparse. Accordingly, in the case of a noiseless signal ( $n=$ $0)$, the received signal $x(t)$ is considered sparse. In the noisy case $(n \neq 0)$, instead of $x$ being exactly sparse (i.e., only $k$ non-zero elements), it is approximately sparse (i.e., only $k$ high amplitude elements). Although we consider random independent noise and fixed target delays, the noise effect can be reduced by integrating the number of PRIs within the CPI. The integration will improve the SNR of the received signal by a factor equal to the number of integrated PRIs [52]. Then, the integrated signal $x_{I}(t)$ can be considered a mostly sparse signal. To simplify notation we will drop the subscript $I$ in our context and use $x(t)$ as the integrated sparse signal.

We can define our CS problem as

$$
y=\Phi_{S} x
$$

where $\Phi_{S}$ is an $M \times N$ matrix composed of selected $M$ rows of the full S-transform matrix defined in (5.8).

Because of the time localization property of the S-transform basis functions, the complete randomness criterion to select the required rows may fail to cover the entire signal. Hence, we have to develop an algorithm to adaptively select the required coefficients (rows).

\subsubsection{Target Range Detection}

The proposed algorithm, Algorithm 2, demonstrates an accurate target range detection using S-transform basis functions in a CS platform. The main concept of the algorithm is to divide the signal into segments. These segments are classified according to their priority.

Since we assume that the target delay is constant during the CPI, we can consider the detected echo delays in the first PRI as prior information for the next PRIs in the same CPI. Accordingly, we can divide the time line of each PRI into segments. The segments are classified into high priority segments where targets are expected (denoted by "target potential") and low priority elsewhere. The proposed S-transform algorithm procedure will follow the same procedures of the wavelet algorithm presented in Chapter. 4 for the target delays detection part.

The algorithm allows for selection of the appropriate bases during the signal sampling process. The selection criterion is influenced by target's delay prior information 
exploiting the multi-resolution basis function's time localization feature. The bases that provide higher time resolution are selected at delays related to the high priority segments. Otherwise, the low priority segments are covered by bases with lower time resolution. That means the entire signal is sampled, but in different resolutions according to the segment's priority classification. In the case of new targets, they will be detected in a lower resolution and marked as a target potential. Then, the algorithm updates the priority segments to include the new delays. Definitely, that will be at the expense of the number of samples.

The algorithm starts by using the Xampling framework to sample the pulse interval $n$ using a subset of S-transform coefficients in lower levels $S_{\left[v, \beta_{c}, \tau_{c}\right]}(t)$ (i.e., coarse resolution)

$$
s_{v, \beta, \tau}=\int_{0}^{T} x(t) S_{[v, \beta, \tau]}(t) d t .
$$

The compressed signal is integrated for I PRIs to improve the SNR. Then, the problem can be formulated as an $\ell_{1}$ optimization problem like (2.9) and the signal can be reconstructed using a CS recovery approach.

Afterwards, as the first step of target detection, the constant false alarm rate (CFAR) approach is applied to provide an adaptive threshold $(\gamma)$. The simplest type of CFAR algorithm, (CA)-CFAR, uses the average received energy in nearby range cells to obtain an adaptive threshold by fixing the probability of false alarm [53]. This adaptive threshold is used for target potential detection after each signal recovery. It is worth mentioning that the CFAR threshold, in this step, is not used to provide complete target detection. It helps to classify the segment's priority into high priority and low priority. Accordingly, the coefficients of higher levels are selected $S_{\left[v, \beta_{f}, \tau_{f}\right]}(t)$ (i.e., fine resolution). In the next PRI, the selected higher and lower level coefficients are merged as

$$
S_{[v, \beta, \tau]}(t)=S_{\left[v, \beta_{c}, \tau_{c}\right]}(t) \cup S_{\left[v, \beta_{f}, \tau_{f}\right]}(t) .
$$

Thus, we can say that the bases are chosen to provide a non-uniform sampling structure to suit the signal nature.

The criterion on which the coefficient should be chosen would be very close to the wavelet coefficients criterion in [54] with a slight difference. In the coarse resolution step, $\beta$ should be chosen such that the basis function's subset covers the entire signal, i.e., $\tau_{c}=1,2, \ldots, \beta_{c}-1$. For fine resolution coefficients, $\beta$ can be chosen such that $\beta=\lceil 2 N / \delta\rceil$, where $\delta$ is the pulse width, to ensure that the time localized window 
is within the pulse width. In the latter case, $\tau$ should be chosen to be consistent with the detected target potential time shifts. To get the best results, $v$ should be optimally chosen such that $v \simeq f_{I F}$ where $f_{I F}$ is the reference IF carrier frequency.

\subsubsection{Doppler Information and CS Moving Target Indicator (CS-MTI)}

Regarding the Doppler frequency information, we use the LCSF as defined in (5.12) [51]. LCSF clearly identifies the phase shift $\phi(\tau, v)$ for a given voice $v$ and time index $\tau$. To measure the phase shift between the transmitted signal and the received echo signal, we consider the transmitted pulse as a reference signal $S_{r e f}(\tau, v)$ and the received echo as $S_{\text {echo }}(\tau, v)$. Hence, the phase shifts of the received target echoes can be calculated using LCSF directly from the compressed measurements as

$$
\phi(\tau, v)=\angle L S C F\left(S_{r e f}(\tau, v), S_{e c h o}(\tau, v)\right) .
$$

Consequently, the Doppler frequency for each target can be estimated by applying a $p$-point discrete Fourier transform (DFT) for each discrete time index $n$ along the PRI. That means that the targets' Doppler frequencies can be detected without the need for a complete and separate CS reconstruction process as done in [36].

Moreover, if we are interested in moving targets only (as many radar applications do), then we can use this step to implement a compressive sensing moving target indicator (CS-MTI). A brief introduction for the concept of the traditional MTI is discussed in Sec. 2.1.1.

The main goal of MTI is to suppress the stationary and slow moving targets. Since we have the ability to estimate the Doppler frequency for the target potentials from the compressed measurements directly and at the same step the algorithm selects the required fine resolution coefficients to allow target detection, we can choose to ignore the target potential delays that have Doppler frequency around zero, i.e., for $-\varepsilon<f_{d}<\varepsilon$, where $\varepsilon$ is a design parameter for the CS-MTI that controls the speed range of the targets of interest. 


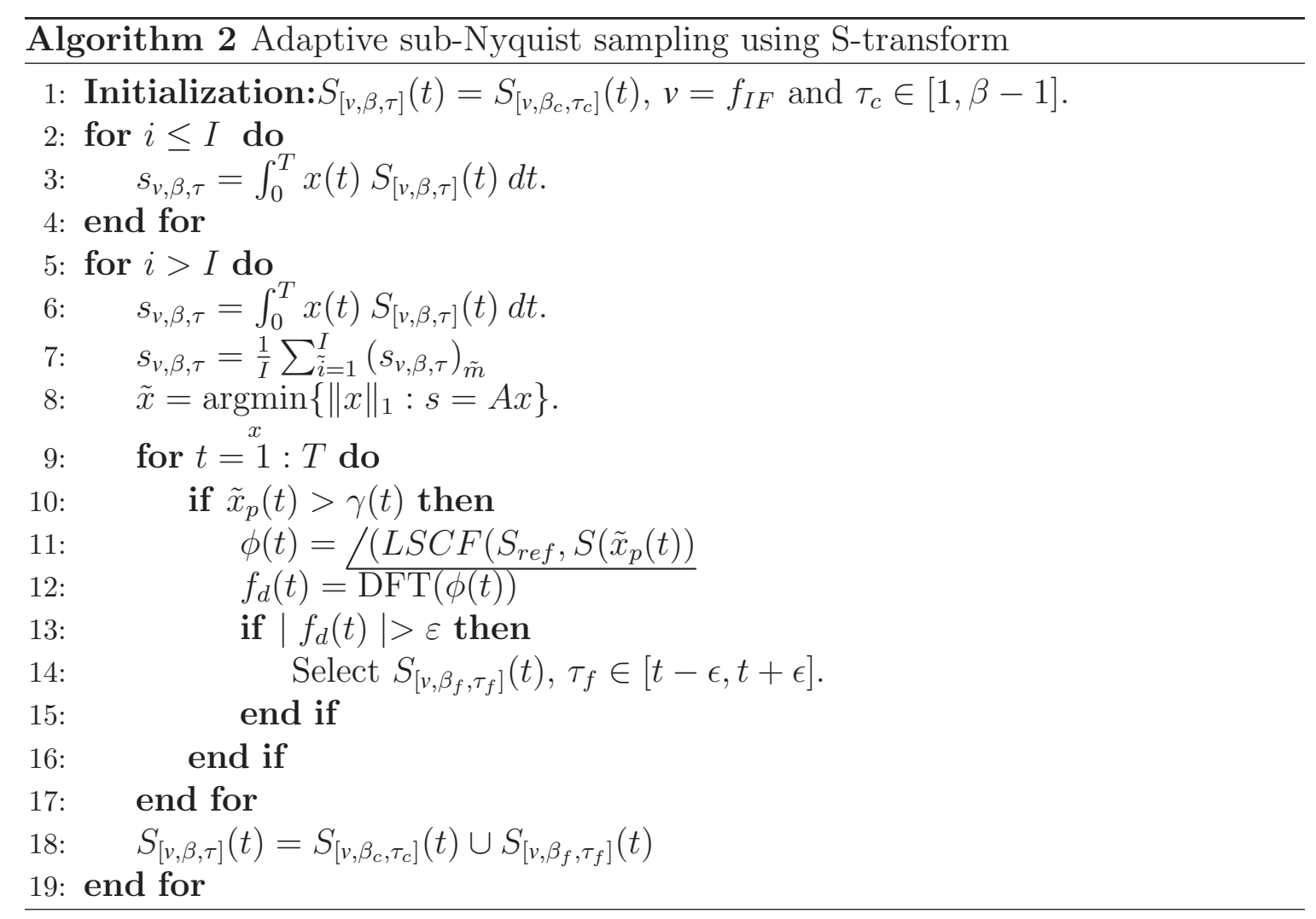

\subsection{Analysis and Results}

In this section, the proposed algorithm performance evaluation is introduced. The performance evaluation is carried out by a comparison between the proposed algorithm, Fourier coefficients approach [4] and the wavelet approach [54]. For completeness, we should also include traditional sampling at a Nyquist rate in the comparison so that one can determine what we gain and forfeit by using one of these sub-Nyquist approaches. We address the probability of detection $\left(P_{d}\right)$ of these approaches in different scenarios. Moreover, in terms of the signal recovery time, we estimate the expected average reconstruction time for the mentioned sub-Nyquist approaches.

\subsubsection{Simulation Parameters}

In our analysis, we simulate a mono-static pulse radar signal with the following parameters. The pulse width $\delta=200 n$ sec. The pulse repetition interval (PRI) $T=100 \mu$ sec. The reference IF is $5 \mathrm{MHz}$. In such cases, to obtain good results, one needs to apply oversampling. That means the number of samples needs to be much more than 
the Nyquist rate which in this case is $10 \mathrm{MHz}$. The simulation was carried out using MATLAB. The signal was represented in 4096 samples. Additive Gaussian noise with zero mean and variance $\sigma^{2}$ was generated and added to the signal. CVX, a package for specifying and solving convex programs [45], was used in the signal reconstruction step. We address the $P_{d}$ in different scenarios at a constant probability of false alarm $\left(P_{f a}\right)$ of $10^{-5}$.

To obtain statistically stable results, 100 Monte Carlo experiments are carried out

for each value of the SNR. The SNR was calculated as $\mathrm{SNR}=\frac{|a|^{2}}{\sigma^{2}}$ where $a^{2}$ is the target peak power.

It is worth noting that the target detection decision of the wavelet and the Stransform algorithms are delayed by $T$ compared to the Fourier and regular Nyquist rate approach. That is because the proposed algorithm and the wavelet algorithm depend on the information from PRI $n-1$ to provide the decision after signal recovery for PRI $n$.

Regarding the number of required coefficients, for both the S-transform approach and the wavelet approach, it varies according to the number of target potentials detected in each pulse interval. However, for the Fourier coefficient approach, there is no clear coefficient selection criterion except the criterion that was used in [4] where a constellation of consecutive coefficients are selected from distributed frequency bands. In our scenario, choosing the Fourier coefficients within the IF bandwidth and the expected Doppler frequencies helps obtain better results. Yet, to have a fair comparison during this experiment, for all sub-Nyquist approaches, the signal was recovered using 256 coefficients which correspond to $25 \%$ of the Nyquist rate samples (1000 samples).

\subsubsection{Results}

The simulation results for all scenarios are depicted using receiver operating characteristic (ROC) curves.

In the first scenario, we simulate a single target in a homogeneous background. The simulation results for this scenario are shown in Fig. 5.1.

In the second scenario, we are testing the reconstruction performance when there are two targets very close in range. Thus, we simulate two targets where the difference between them is only one range cell. The ROC curves in Fig. 5.2 shows the simulation results for this scenario. 


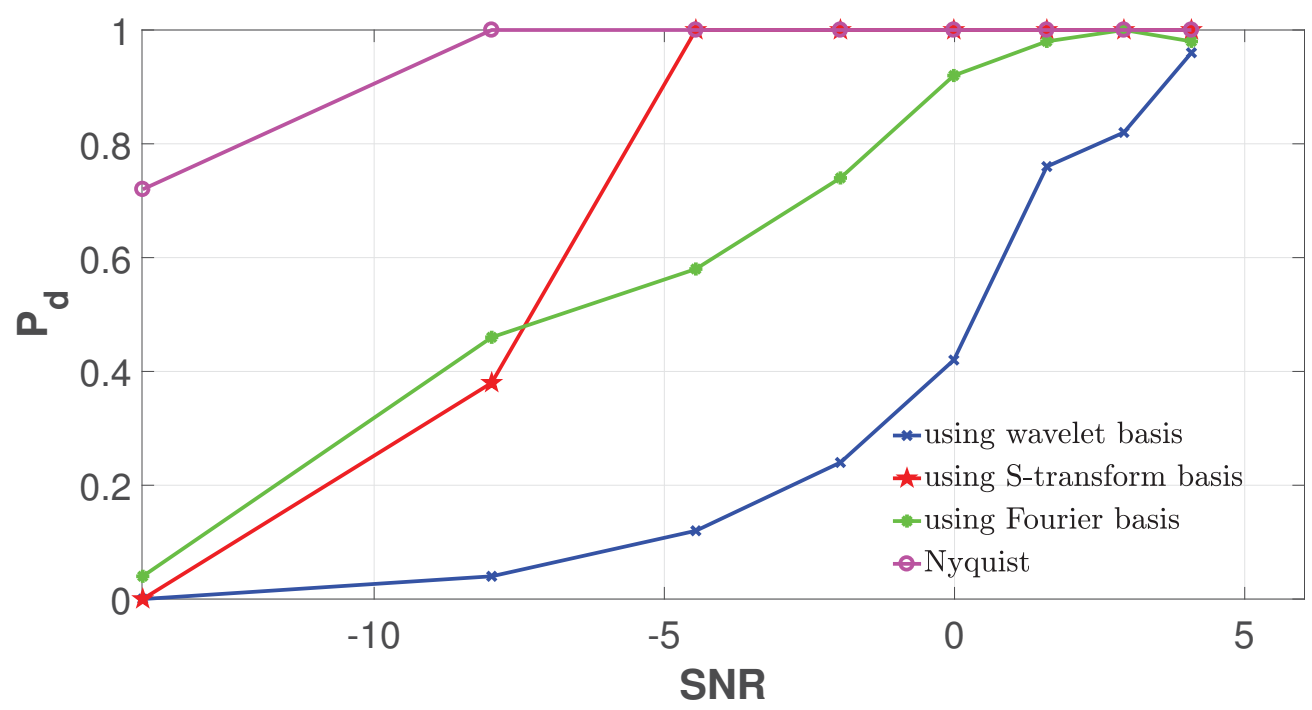

Figure 5.1: ROC curve for a simulation of a single target in a homogeneous background using different basis functions $\left(P_{f a}=10^{-5}\right)$.

In the third scenario, we simulate a target in a Rayleigh distributed clutter zone. Figure 5.3 shows the simulation results for the third scenario.

To evaluate $P_{d}$, we used a cell average CFAR (CA-CFAR) detector in the first and third scenarios. However, in the second scenario, we used the smallest of CFAR (SO-CFAR) detector because its performance is better when two targets are very close [53].

As a metric of complexity and signal reconstruction speed, we used the average reconstruction time, estimated using the CVX parameter "cvx_cputime", to compare between the studied sub-Nyquist approaches using different number of coefficients. The results are presented in Table 5.1.

\subsubsection{Discussion}

For the first and second scenarios, Figures 5.1 and 5.2 show an improvement in $P_{d}$ using the proposed algorithm for different SNR values. In the third scenario, Fig. 5.3 , the Fourier coefficient approach shows better $P_{d}$ than the proposed algorithm for the lower values of the SNR. However, the performance for both is similar for higher SNR values. 


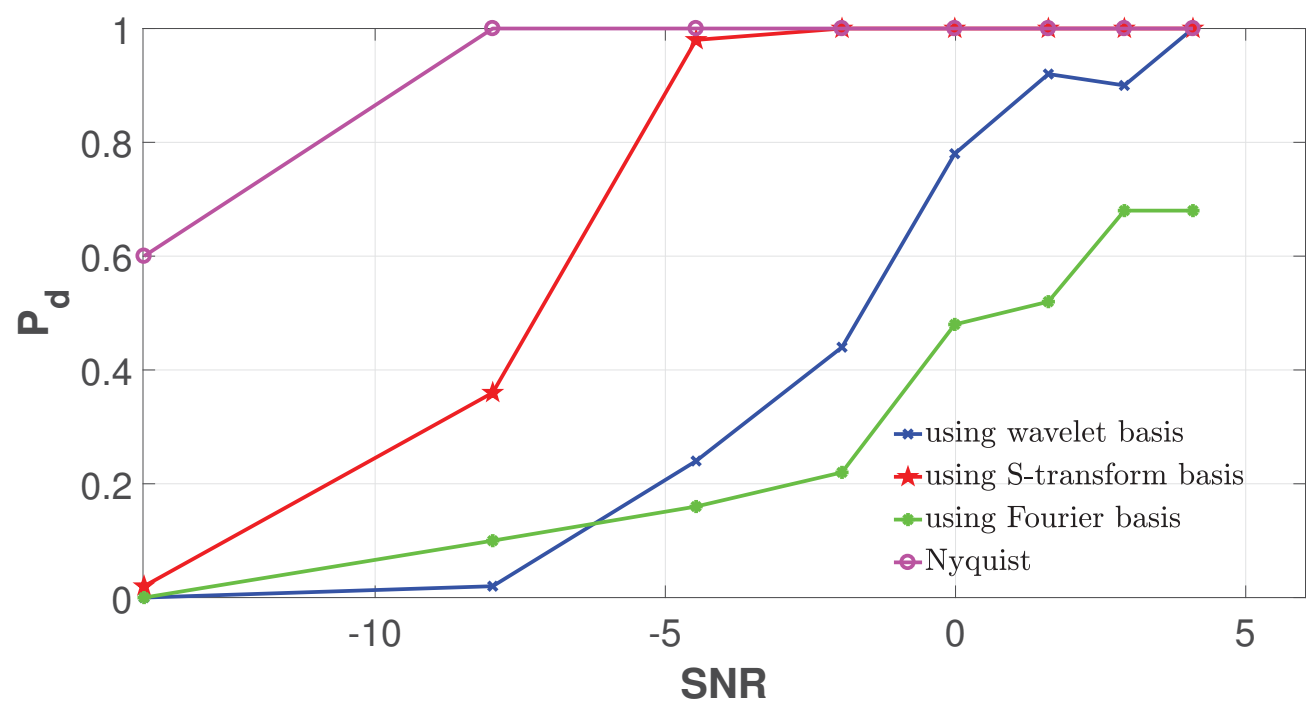

Figure 5.2: ROC curve for a simulation of two targets in a homogeneous background using different basis functions $\left(P_{f a}=10^{-5}\right)$.

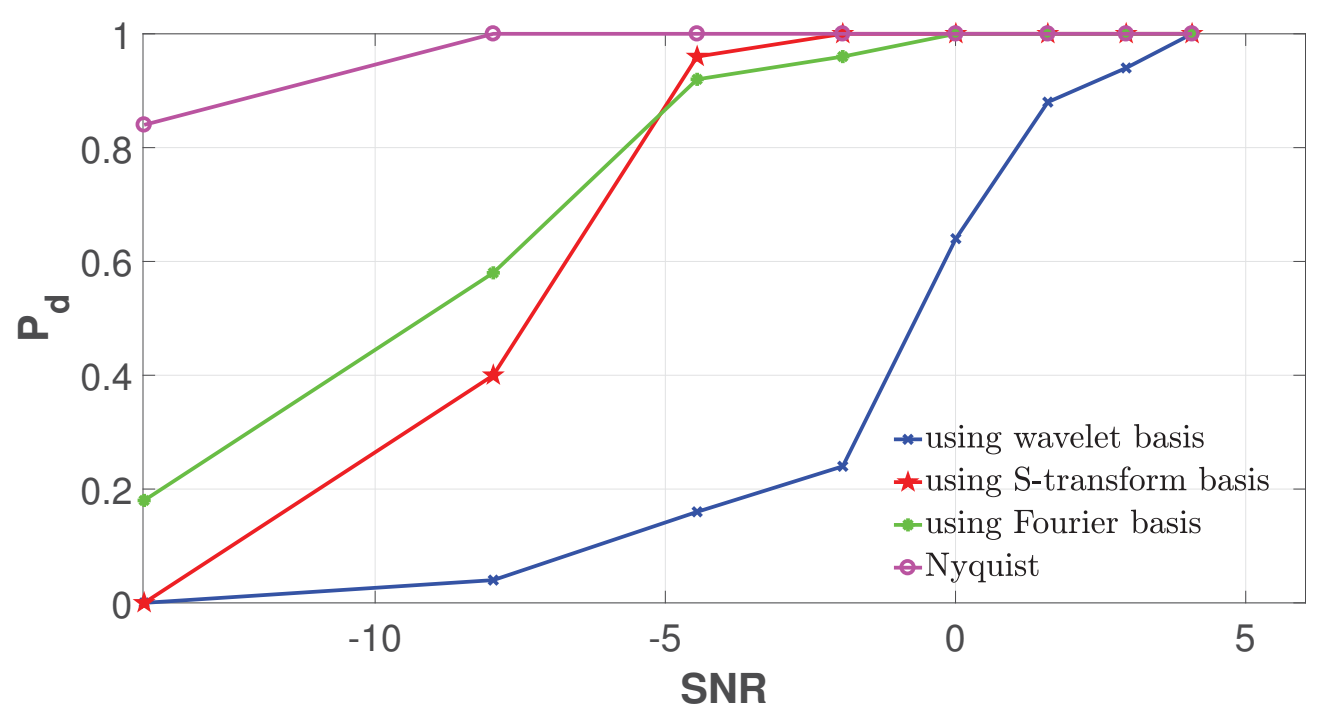

Figure 5.3: ROC curve for a simulation of a target in a clutter zone using different basis functions $\left(P_{f a}=10^{-5}\right)$. 
Table 5.1 shows that the recovery time for the proposed S-transform algorithm approach slightly exceeds the wavelet approach. However, that difference might be considered reasonable compared to the benefits that this approach introduces (Doppler frequency information extraction). Moreover, the recovery time using the S-transform approach is still better than the Fourier approach's time recovery. For example, the proposed algorithm is 187 times faster when 256 coefficients are selected and reaches about 600 times faster when 512 coefficients are selected.

Table 5.1: Recovery statistics for different number of coefficients

\begin{tabular}{cccc}
\hline & \multicolumn{3}{c}{ Recovery time (sec) } \\
\cline { 2 - 4 } No. Coefficients & $\begin{array}{c}\text { Fourier } \\
\text { bases }\end{array}$ & $\begin{array}{c}\text { Wavelet } \\
\text { bases }\end{array}$ & $\begin{array}{c}\text { S-transform } \\
\text { bases }\end{array}$ \\
\hline 32 & 3.9205 & 0.4986 & 0.7400 \\
64 & 12.0261 & 0.5106 & 0.7667 \\
128 & 46.088 & 0.5283 & 0.8003 \\
256 & 164.2539 & 0.6814 & 0.8789 \\
512 & 605.6850 & 0.7684 & 1.0148 \\
\hline
\end{tabular}

\subsection{Conclusion}

In this chapter, we proposed a compressive sensing based algorithm using S-transform basis functions for sub-Nyquist sampling of pulsed radar signals. The proposed algorithm is able to extract target range and Doppler frequency information using a much lower number of samples than the Nyquist rate. A performance evaluation of the proposed algorithm was presented and compared with competing approaches in different target scenarios. The simulation results show that the proposed algorithm can be about 600 times faster than the Fourier coefficients approach and that gap increases dramatically when the number of coefficients increases. Moreover, it achieves reasonable improvement in the target detection probability $P_{d}$ for different scenarios. However, the recovery time for the proposed algorithm is longer than the Haar wavelet algorithm that allows for target range detection only. 


\section{Chapter 6}

\section{Recovery Guarantees for S-transform Matrix}

In this chapter, we introduce a mathematical proof for the recovery guarantees of the proposed S-transform algorithm discussed in Chapter 5. Our aim is to prove an upper bound on the number of detectable targets in the presence of additive noise. For completeness of this chapter, we will start by representing a mathematical background on compressive sensing then proceed to the recovery guarantees proof.

\subsection{Mathematical background on compressive sensing}

The basic model for the pulse radar problem we are considering involves a linear measurement equation

$$
y=\Phi x
$$

where $y$ is an $M \times 1$ vector of measurements collected by the receiver antenna over an observation interval, $\Phi$ is an $M \times N$ measurement matrix and $x$ is an $N \times 1$ vector whose largest elements represent the received target signal echoes. The solution of this undetermined problem can be achieved by solving a convex optimization problem such as $\ell_{1}$ norm, which is a linear optimization problem and can be expressed as

$$
\hat{x}_{1}=\underset{x}{\arg \min }\|x\|_{1} \quad \text { s.t. } \quad y=\Phi x
$$


where $\hat{x}_{1}$ is the sparsest solution that satisfies $y=\Phi \hat{x}_{1}$. This problem is called basis pursuit (BP) [22].

As an alternative to $\ell_{1}$ relaxation, [23] introduced the FOCUSS algorithm that employs $\ell_{p}$ norm instead of $\ell_{0}$ for $0<p<1$. Although this problem is non-convex, FOCUSS provides an iterative algorithm to solve the optimization problem.

The second way to solve the CS problem is through greedy algorithms, such as matching pursuit (MP) [24], orthogonal matching pursuit (OMP) [25] and compressive sampling matching pursuit (CoSaMP) [28]. CoSaMP provides better stability and robustness results. For sufficiently small worst case coherence $\mu_{\Phi}$, [26] shows that OMP can recover $k$-sparse signal support with high probability. Moreover, these results are further studied and improved in [27] to show that the signal can be recovered using only $\mathcal{O}(k \log N)$ measurements.

Another set of algorithms, which could be shown to efficiently solve the CS problem are the thresholding-based methods such as basic thresholding and iterative hard thresholding $[55,56]$. As a combination of the Iterative Hard Thresholding algorithm and CoSaMP, [57] introduced a new low complexity iterative algorithm called Hard Thresholding Pursuit.

That leads us to the result that as long as $k=\mathcal{O}\left(\mu_{\Phi}^{-1}\right)$, an under-determined system of equation $y=\Phi x$ for a $k$-sparse signal can be solved in polynomial time [5].

To consider the measurement noise $n$ added to the signal, a small modification can be made to (6.2) to express the optimization problem for a noisy case to be expressed as

$$
\hat{x}_{1}=\underset{x \in \mathscr{H}^{M}}{\arg \min }\|x\|_{1} \quad \text { s.t } \quad\|y-\Phi x\|_{2} \leq \epsilon
$$

where $\epsilon=\|n\|$, i.e., it is typically chosen to be equal to the noise magnitude. This modified version is now called basis pursuit with inequality constraint (BPIC) [5]. Regarding OMP, the same algorithm should work without modification except for the value of $\epsilon=\|n\|$ instead of 0 in the former case.

It is worthwhile noting that in the noisy case, for all methods, the recovered signal will be considered an estimation for the sparse signal.

This noisy model in (6.3) is considered as a deterministic model. If we wish to deal with stochastic noise such as additive white Gaussian noise (AWGN), then (6.3) can be modified as 


$$
\hat{x}=\underset{x \in \mathscr{H}^{M}}{\arg \min } \frac{1}{2}\|y-\Phi x\|_{2}^{2}+\lambda\|x\|_{1}
$$

where $\lambda$ is a regularization parameter that controls the trade off between error and the sparsity of the solution and is chosen based on empirical considerations. This optimization problem is called basis pursuit denoising (BPDN) [22] or least absolute shrinkage and selection operation (LASSO) [5].

Recovery of the signal using the mentioned algorithms is guaranteed with high probability under some conditions on the measurement matrix $\Phi$ such as satisfying the coherence condition and the restricted isometry property (RIP).

In the recovery algorithm analysis, one needs a measure of the suitability of the measurement matrix. One of the simplest such measures is the coherence. The minimum and satisfactory condition for signal sparsity is directly related to the measurement matrix coherence and is provided by the following theorem.

Theorem 2. [5, 58,59]. An arbitrary $k$-sparse signal $x$ can be uniquely recovered from $y=\Phi x$ as a solution to an $\ell_{1}$ minimization problem provided

$$
k<\frac{1}{2}\left(1+\mu_{\Phi}^{-1}\right)
$$

where $\mu_{\Phi}$ is the worst-case coherence which expresses the measure of the similarity between different frame elements [5], and can be defined as

$$
\mu_{\Phi}=\max _{\substack{i, j \in\{1, \ldots, m\} \\ i \neq j}} \frac{\left|\left\langle\varphi_{i}, \varphi_{j}\right\rangle\right|}{\left\|\varphi_{i}\right\|_{2}\left\|\varphi_{j}\right\|_{2}} .
$$

where $\varphi_{i}$ is the ith column of the matrix $\Phi$.

This theorem defines the relation between signal sparsity upper bound and the coherence of the measurement matrix columns. It shows that the maximum number of the signal's nonzero elements that guarantee signal reconstruction with high probability is inversely proportional with the maximum coherence between any two columns of the measurement matrix. In other words, the smaller the coherence, the better the signal reconstruction performance.

Piya Pal and P. P. Vaidyanathan in [60] used (6.5) in their recovery guarantees. They show an improvement in the maximum level of sparsity to $\mathcal{O}\left(M^{2}\right)$ instead of $\mathcal{O}(M)$ by using the correlation structure of the measurements. 
On the other hand, Strohmer and Friedlander [61,62], follow Candès and Plan [63], to provide bounds on the recovery guarantees and the maximum number of detectable targets using measurement matrix mutual coherence and operator norm bounds based on the following theorem.

Theorem 3. (Theorem (1.2) in [63] and its extended version Theorem (15) in [61])

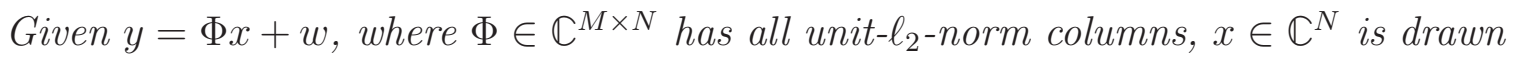
from the generic $K$-sparse model and $w_{i} \in \mathbb{C} N\left(0, \sigma^{2}\right)$. Assume that

$$
\mu_{\Phi} \leq \frac{C_{0}}{\log N}
$$

where $C_{0}$ is a constant independent on $M, N$. Furthermore, suppose

$$
K \leq \frac{C_{0} N}{\|\Phi\|_{o p}^{2} \log N}
$$

for some constant $C_{0} \geq 0$ and that

$$
\min _{k \in K}\left|x_{k}\right|>8 \sigma \sqrt{2 \log N}
$$

Then the solution $\hat{x}$ to the LASSO computed with $\lambda=2 \sigma \sqrt{2 \log N}$ obeys

$$
\operatorname{supp}(\hat{x})=\operatorname{supp}(x)
$$

and

$$
\frac{\|\hat{x}-x\|_{2}}{\|x\|_{2}} \leq \frac{\sigma \sqrt{3 M}}{\|y\|_{2}}
$$

with probability at least

$$
1-2(N)^{-1}\left\{2 \pi \log N+K(N)^{-1}\right\}-\mathcal{O}\left(N^{-2 \log 2}\right)
$$

where $\|\Phi\|_{o p}$ denotes the operator norm of matrix $\Phi$ and $\hat{x}$ denotes the recovered signal.

A finer measure for the suitability of the measurement matrix is RIP which can be defined as follows.

Definition 2. [5] (Restricted Isometry Property (RIP))

A unit norm frame $\Phi=\left(\varphi_{i}\right)_{i=1}^{M}$ in Hilbert space $\mathscr{H}^{N}$ is said to have the RIP of order $k$ with parameter $\delta_{k} \in(0,1)$ if for every $k$-sparse $x$, the following inequalities hold: 
Table 6.1: Support recovery guarantees for different measurement matrices

\begin{tabular}{cccc}
\hline \multicolumn{1}{c}{ Matrix Type } & Mutual Coherence & Sparsity & References \\
\hline $\begin{array}{l}\text { Random matrix with }(N M) \\
\text { independent random entries }\end{array}$ & $\mathcal{O}\left(2 \sqrt{\frac{\log N}{M}}\right)$ & $\mathcal{O}\left(\frac{M}{\log N}\right)$ & {$[65][66]$} \\
\hline $\begin{array}{l}\text { Toeplitz block matrix with } \\
(N+M) \text { random entries }\end{array}$ & $\mathcal{O}\left(\sqrt{\frac{\log N}{M}}\right)$ & $\mathcal{O}\left(\frac{M}{\log N}\right)$ & \\
\hline & $\mathcal{O}\left(\sqrt{\frac{\log N}{M \log M}}\right)$ & $\mathcal{O}\left(\frac{M \log M}{\log N}\right)$ & Current work \\
\hline & & & \\
\hline
\end{tabular}

$$
\left(1-\delta_{k}\right)\|x\|_{2}^{2} \leq\|\Phi\|_{2}^{2} \leq\left(1+\delta_{k}\right)\|x\|_{2}^{2}
$$

Rahaut [64] introduced deeper analysis and a new bound on the number of measurements needed for accurate recovery relying on the RIP of the measurement matrix.

In our work, we will use measurement matrix mutual coherence to find the upper bound for the number of detectable targets as in (6.5).

Table 6.1 summarizes related results for support recovery for different measurement matrices.

\subsection{Signal model}

We consider a problem of a mono-static pulse radar. The transmitted signal is a rectangular shaped pulse with a duration $\delta$ and a constant intermediate frequency $F_{I F}$ during the pulse duration. Each target is defined by three parameters. Time delay $\left(t_{d}\right)$ directly related to target range, Doppler frequency $\left(f_{d}\right)$ directly related to target speed and scatter coefficient $a$ related to radar target cross-section and propagation factors . 
The transmitted pulse is repeated periodically every pulse repetition interval $(\mathrm{PRI}=T)$. The reciprocal of the $\mathrm{PRI}$ is the pulse repetition frequency $(\mathrm{PRF}=1 / \mathrm{PRI})$.

We consider a coherent pulse interval (CPI) that involves $I$ PRIs. During one CPI, we consider the same assumptions as stated in Sec. 5.3.1.

Let $x(t)$ represent the received signal for one PRI. Then

$$
x(t)=\sum_{p=1}^{N} a_{p} h(t-p) e^{-j 2 \pi f_{d} t}
$$

where $h(t)$ is the transmitted pulse and $N=T / \delta$ is the number of range cells.

The scatter coefficients vector $\left\{a_{i}: i \in\{1, \ldots, N\}\right\}$ has values at echo delays only and zero otherwise. Since we assume that the number of targets is much less than the number of range cells, then

$$
\|a\|_{0}:=\sum_{i=1}^{N} 1_{\left\{a_{i} \neq 0\right\}}(x)=k \ll N .
$$

Hence, $a_{i}$ is considered sparse. Accordingly, the received signal $x(t)$ is considered sparse. We can define our CS problem as

$$
y=\Phi_{S} x
$$

where $\Phi_{S}$ is an $M \times N$ matrix composed of selected $M$ rows of the full S-transform matrix.

\subsection{Main Result}

The main result in this chapter is the recovery guarantees upper bound for the signal sparsity and can be represented in the next theorem.

Theorem 4. Consider $y=\Phi_{S} x$, where $\Phi_{S}$ is an $M \times N$ matrix composed of $M$ rows of the $N^{2} \times N$ S-transform matrix. If $x$ is drawn from the generic $k$-sparse target model with

$$
K \leq \frac{1}{2}+\sqrt{\frac{M \log M}{2 \log N}}
$$

Then $x$ can be uniquely recovered with probability at least 


$$
1-2 N^{-c \frac{N^{2}}{M^{2}} \log M}-2 N^{-c \frac{M}{\log M}}
$$

\section{Remarks}

i) The upper bound for the maximum number of detectable targets is found to be reasonable compared to the bounds in the literature for different types of matrices as shown in Table 6.1.

ii) The individual terms in the expression of the probability of success are fairly small. Hence, this bound provides unique signal recovery with very high probability.

\subsection{Proof of Theorem 4}

Before starting the proof of Theorem 4, we will state two Lemmas to be used in the proof.

\subsubsection{Useful Lemmas}

Since our aim is to find a bound for the coherence $\mu_{\Phi}$ that leads us to find the upper bound on the sparsity $k$, which represents the number of targets in our context, we will use a modified version of Hanson-Wright inequality on tail bounds for quadratic forms [68] but for the case of Bernoulli random variables [69].

Lemma 1. [69] Let $J$ be a random subset of $[n]$ of size $M<N$ uniformly chosen among all such subsets. Denote by $R_{j}=\sum_{j \in J} e_{j} e_{j}^{T}$ the coordinate projection on the set $J$ where $e_{j}$ refers to the standard basis (row) vector with a one in the $j^{\text {th }}$ component and zero elsewhere. Let $Y=\left(\varepsilon_{1}, \ldots, \varepsilon_{N}\right)$ be the vector whose coordinates are independent symmetric \pm 1 Bernoulli random variables. Then for any $N \times N$ matrix $A$ and any $t>0$,

$$
\begin{gathered}
\mathbb{P}\left(\left|Y^{T} R_{J} A R_{J} Y-\mathbb{E} Y^{T} R_{J} A R_{J} Y\right| \geq t\right) \\
\quad \leq 2 \exp \left[-c \min \left(\frac{t^{2}}{M\|A\|^{2}}, \frac{t}{\|A\|}\right)\right]
\end{gathered}
$$

Proof. The proof of this lemma can be found in [69]. 
The next Lemma will be used in the S-transform columns norm estimation.

Lemma 2. The sum of the Gaussian series

$$
\sum_{x=0}^{N-1} \exp \left[-a\left(\frac{x}{N}\right)^{2}\right]=\frac{N}{2} \sqrt{\frac{\pi}{a}} \operatorname{Erf}(\sqrt{a}) .
$$

for any $a>0$ and large enough $N$, where $\operatorname{Erf}(\cdot)$ is the error function.

Proof. Let us consider a function $f(z)=\exp \left[-a z^{2}\right]$ on an interval $[c, d]$ is divided into $N$ equal intervals of width $\Delta z$. Let $c=0$ and $d=1$. Hence

$$
\Delta z=\frac{d-c}{N}=\frac{1}{N}
$$

and if

$$
z_{x}=c+x \Delta z=\frac{x}{N}
$$

is the right endpoint of sub-interval $x$, then

$$
f\left(z_{x}\right)=\exp \left[-a\left(\frac{x}{N}\right)^{2}\right]
$$

The Riemann sum of $\mathrm{f}$ over the interval $[c, d]$ with partition $\Delta z$ is defined as

$$
\int_{c}^{d} f(z) d z=\lim _{N \rightarrow \infty} \sum_{x=1}^{N} f\left(z_{x}\right) \Delta z
$$

Substituting from (6.19) and (6.21) in (6.22)

$$
\int_{0}^{1} \exp \left[-a z^{2}\right] d z=\lim _{N \rightarrow \infty} \frac{1}{N} \sum_{x=1}^{N} \exp \left[-a\left(\frac{x}{N}\right)^{2}\right]
$$

Inversely, for large $N$

$$
\sum_{x=1}^{N} \exp \left[-a\left(\frac{x}{N}\right)^{2}\right]=N \int_{0}^{1} \exp \left[-a z^{2}\right] d z .
$$

However,

$$
\int_{0}^{\sqrt{a}} \exp \left[-a t^{2}\right] d t=\frac{1}{2} \sqrt{\frac{\pi}{a}} \operatorname{Erf}(\sqrt{a}) .
$$


From (6.24) and (6.25), hence

$$
\sum_{x=1}^{N} \exp \left[-a\left(\frac{x}{N}\right)^{2}\right]=\frac{N}{2} \sqrt{\frac{\pi}{a}} \operatorname{Erf}(\sqrt{a})
$$

for any $a>0$ and large enough $N$, which concludes the proof.

\subsubsection{Theorem 4 proof}

Proof. Since we consider a measurement matrix that is composed of selected rows from the complete S-transform matrix, we should first study the nature of the Stransform matrix which can be represented as

$$
S=\left[\begin{array}{cccc}
s_{1,1}(1) & s_{1,1}(2) & \cdots & s_{1,1}(N) \\
s_{1,2}(1) & s_{1,2}(2) & \cdots & s_{1,2}(N) \\
\vdots & \vdots & \cdots & \vdots \\
s_{1, N}(1) & s_{1, N}(2) & \cdots & s_{1, N}(N) \\
s_{2,1}(1) & s_{2,1}(2) & \cdots & s_{2,1}(N) \\
\vdots & \vdots & \cdots & \vdots \\
s_{N, N}(1) & s_{N, N}(2) & \cdots & s_{N, N}(N)
\end{array}\right]
$$

where

$$
s_{\tau, f}(n)=e^{-\frac{f^{2}(\tau-n)^{2}}{2 N^{2}}} e^{-j 2 \pi \frac{f n}{N}} .
$$

It is clear from the structure of the S-transform basis function that it is composed of two main sections. The first one is the time localization section which is represented by a Gaussian window. The second section can be shown as a regular Fourier series which is responsible for retaining the advantageous phase properties of the S-transform. Hence. we can represent the S-transform matrix as a Hadamard product of two matrices, a Gaussian window matrix $G$ and discrete Fourier transform matrix $F$ as

$$
S=G \circ F .
$$

where $\circ$ is an element-wise multiplication. 
Hence, we need two key estimates: one concerns a bound for the coherence of matrix $G$, the other one concerns a bound for the coherence of matrix $F$. We can divide our problem into two parts: the Gaussian window part and the DFT matrix part.

Accordingly, the proof is carried out in two steps. In the first step, we will deal with a Gaussian window matrix $G$ which is sub-sampled in window shifts $\tau$. In the second part, we will deal with a DFT matrix $F$ which is sub-sampled in frequencies. Then we combine the two parts to construct an S-transform matrix that is sub-sampled in frequencies and window shifts.

\section{Part 1. Gaussian window matrix $G$}

The worst-case coherence in (6.6) requires the matrix to have columns of unitnorm, whereas the columns of matrix $G$ have all different norms. Hence, as learned from [61], instead of $y=\Phi x$, we can consider

$$
y=\tilde{G} z
$$

where $\tilde{G}=G D^{-1}, z=D x$ and $D$ is the $N \times N$ diagonal matrix defined by

$$
D_{i i}=\left\|g_{i}\right\|_{2} \text {. }
$$

where $g_{i}$ is column $i$ in $G$.

Let's consider $p_{i}$ is the norm of column $g_{i}$ in $G$, i.e., $\left\|g_{i}\right\|_{2}=p_{i}$. Then,

$$
D=\left[\begin{array}{cccc}
p_{1} & 0 & \cdots & 0 \\
0 & p_{2} & & \vdots \\
\vdots & & \ddots & 0 \\
0 & \cdots & 0 & p_{N}
\end{array}\right]
$$


Hence, the normalized matrix $\tilde{G}$ can be represented as

$$
\tilde{G}=G D^{-1}=\left[\begin{array}{cccc}
\frac{g_{1,1}(1)}{p_{1}} & \frac{g_{1,1}(2)}{p_{2}} & \ldots & \frac{g_{1,1}(N)}{p_{N}} \\
\frac{g_{1,2}(1)}{p_{1}} & \frac{g_{1,2}(2)}{p_{2}} & \ldots & \frac{g_{1,2}(N)}{p_{N}} \\
\vdots & \vdots & \ldots & \vdots \\
\frac{g_{1, N}(1)}{p_{1}} & \frac{g_{1, N}(2)}{p_{2}} & \ldots & \frac{g_{1, N}(N)}{p_{N}} \\
\frac{g_{2,1}(1)}{p_{1}} & \frac{g_{2,1}(2)}{p_{2}} & \ldots & \frac{g_{2,1}(N)}{p_{N}} \\
\vdots & \vdots & \ldots & \vdots \\
\frac{g_{N, N}(1)}{p_{1}} & \frac{g_{N, N}(2)}{p_{2}} & \ldots & \frac{g_{N, N}(N)}{p_{N}}
\end{array}\right]
$$

and the $i$ th column is represented as

$$
\tilde{g}_{i}=\left[\begin{array}{c}
\frac{g_{1,1}(i)}{p_{i}} \\
\frac{g_{1,2}(i)}{p_{i}} \\
\vdots \\
\frac{g_{1, N}(i)}{p_{i}} \\
\frac{g_{2,1}(i)}{p_{i}} \\
\vdots \\
\frac{g_{N, N}(i)}{p_{i}}
\end{array}\right] .
$$


In order to select $M$ out of $N$ rows, we can represent $\tilde{g}_{i}=\grave{g}_{i} R_{J} \mathbb{1}$ as

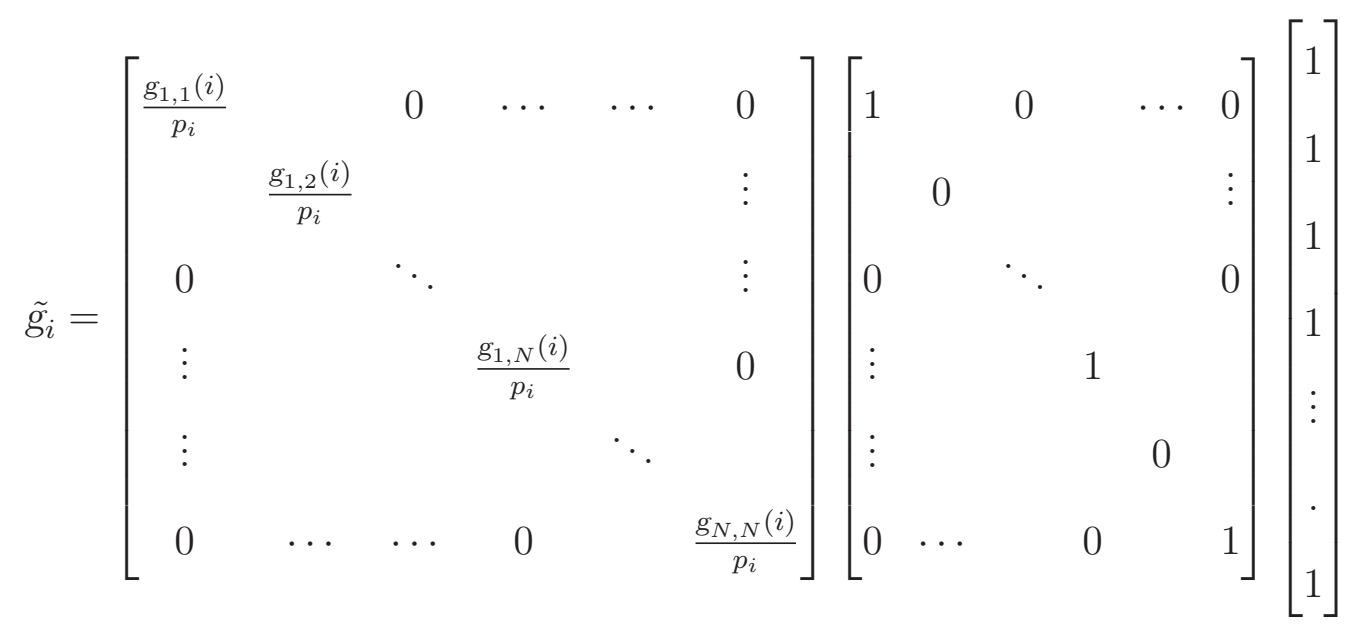

where $R_{J}$ is as defined in Lemma 1 and $\mathbb{1}$ is a unity vector.

Then, the inner product in (6.6) is

$$
\begin{aligned}
\left\langle\tilde{g}_{i}, \tilde{g}_{j}\right\rangle & =\tilde{g}_{i}^{H} \tilde{g}_{j} \\
& =\left(\grave{g}_{i} R_{J} \mathbb{1}\right)^{H}\left(\grave{g}_{j} R_{J} \mathbb{1}\right) \\
& =\left(\mathbb{1}^{T} R_{J} \grave{g}_{i}^{H} \grave{g}_{j} R_{J} \mathbb{1}\right) \\
& =\mathbb{1}^{T} R_{J} A_{G} R_{J} \mathbb{1}
\end{aligned}
$$

where

$$
\begin{aligned}
A_{G} & =\grave{g}_{i}^{H} \grave{g}_{j} \\
& =\operatorname{diag}\left[\frac{g_{1,1}(i) g_{1,1}(j)}{p_{i} p_{j}} \cdots \frac{g_{N, N}(i) g_{N, N}(j)}{p_{i} p_{j}}\right]
\end{aligned}
$$

and

$$
g_{\tau, f}(n)=e^{\frac{-f^{2}(\tau-n)^{2}}{2 N^{2}}} .
$$

By changing $\mathbb{1}$ with $Y$ in $(6.36)$, the result will remain the same since the negative values in the Bernoulli vector $Y$ in both sides will cancel each other. Hence, we can apply Lemma 1 to find the coherence bound.

To apply Lemma 1, we need to find the key estimate, the operator norm of $A_{G}$ as

$$
\left\|A_{G}\right\|=\sqrt{\lambda_{\max }\left(A_{G}^{H} A_{G}\right)} .
$$


where $\lambda_{\max }\left(A_{G}^{H} A_{G}\right)$ is the eigenvalue of matrix $A_{G}^{H} A_{G}$. Since

$$
A_{G}^{H} A_{G}=\operatorname{diag}\left[\left(\frac{g_{1,1}(i) g_{1,1}(j)}{p_{i} p_{j}}\right)^{2}, \cdots,\left(\frac{g_{N, N}(i) g_{N, N}(j)}{p_{i} p_{j}}\right)^{2}\right]
$$

then

$$
\lambda_{\max }\left(A_{G}^{H} A_{G}\right)=\max _{\substack{i, j \in\{1, \ldots, N\} \\ i \neq j}}\left(\max _{\tau, f \in\{1, \ldots, N\}}\left(\frac{g_{\tau, f}(i) g_{\tau, f}(j)}{p_{i} p_{j}}\right)^{2}\right) .
$$

From (6.38) and (6.41),

$$
\begin{aligned}
\lambda_{\max }\left(A_{G}^{H} A_{G}\right) & =\max _{\substack{i, j \in\{1, \ldots, N\} \\
i \neq j}}\left(\frac{1}{p_{i}^{2} p_{j}^{2}} \max _{\tau, f \in\{1, \ldots, N\}}\left(e^{\frac{-f^{2}(\tau-i)^{2}}{N^{2}}} e^{\frac{-f^{2}(\tau-n)^{2}}{N^{2}}}\right)\right) \\
& =\max _{\substack{i, j \in\{1, \ldots, N\} \\
i \neq j}}\left(\frac{1}{p_{i}^{2} p_{j}^{2}} \max _{\tau, f \in\{1, \ldots, N\}}\left(e^{-\left(\frac{f^{2}\left((\tau-i)^{2}+(\tau-j)^{2}\right)}{N^{2}}\right)}\right)\right) .
\end{aligned}
$$

At this point, we have two maximization problems to solve. The inner maximization is in the form of max $e^{-x}$. Solving this problem is equal to finding the minimum of $x$. Then, the inner maximum problem can be simplified to

$$
\max _{\tau, f \in\{1, \ldots, N\}}\left(e^{-\left(\frac{f^{2}\left((\tau-i)^{2}+(\tau-j)^{2}\right)}{N^{2}}\right)}\right) \cong \min _{\tau, f \in\{1, \ldots, N\}}\left(f^{2}\left((\tau-i)^{2}+(\tau-j)^{2}\right)\right)
$$

Excluding the trivial solution at zero, the minimum can be simply found at

$$
f=1 \quad \text { and } \quad \tau=\frac{i+j}{2}
$$

Hence (6.42) reduces to the maximization problem

$$
\lambda_{\max }\left(A_{G}^{H} A_{G}\right)=\max _{\substack{i, j \in\{1, \ldots, N\} \\ i \neq j}}\left(\frac{e^{-\frac{(i-j)^{2}}{2 N^{2}}}}{p_{i}^{2} p_{j}^{2}}\right) .
$$

To solve (6.45), we can split it into two minimization problems as 


$$
\begin{gathered}
\min _{\substack{i, j \in\{1, \ldots, N\} \\
i \neq j}}\left((i-j)^{2}\right), \\
\min _{\substack{i, j \in\{1, \ldots, N\} \\
i \neq j}}\left(p_{i}^{2} p_{j}^{2}\right) .
\end{gathered}
$$

Clearly,

$$
j=i \pm 1
$$

should solve (6.46a). Accordingly, we need to find the minimum norms for any two consecutive columns in the Gaussian window matrix $G$ to solve (6.46b).

To solve (6.46b), we have to study the behavior of matrix $G$ columns norm $p_{i}$ which can be expressed as

$$
\begin{aligned}
p_{i}^{2} & =\sum_{\tau=0}^{N-1} \sum_{f=0}^{N-1}\left|g_{\tau, f}(i)\right|^{2} \\
& =g_{0,0}^{2}(i)+g_{0,1}^{2}(i)+\cdots+g_{0, N-1}^{2}(i)+g_{1,0}^{2}(i)+\cdots+g_{M-1, N-1}^{2}(i) .
\end{aligned}
$$

Recalling

$$
g_{\tau, f}^{2}(i)=\exp \left[-\frac{(\tau-i)^{2} f^{2}}{N^{2}}\right]
$$

then, (6.48) can be expressed as

$$
p_{i}^{2}=\sum_{a} \sum_{f=0}^{N-1} \exp \left[-a\left(\frac{f}{N}\right)^{2}\right]
$$

where $a=(\tau-i)^{2}$.

Using Lemma 2, the inner sum in (6.50) can be simplified to

$$
p_{i}^{2}=\sum_{a} \frac{N}{2} \sqrt{\frac{\pi}{a}} \operatorname{Erf}(\sqrt{a}) .
$$

We claim that

$$
p_{i}^{2}<p_{i+1}^{2} \quad \text { for } \quad i<\frac{N}{2} \quad \text { and } \quad p_{i}^{2}>p_{i+1}^{2} \quad \text { for } \quad i>\frac{N}{2} \text {. }
$$


To verify this claim, we recall that the structure of $p_{i}$ is

$$
\begin{aligned}
p_{i}^{2}= & \sum_{f=0}^{N-1} \exp \left[i^{2} \frac{f^{2}}{N^{2}}\right]+\sum_{f=0}^{N-1} \exp \left[(1-i)^{2} \frac{f^{2}}{N^{2}}\right]+\sum_{f=0}^{N-1} \exp \left[(2-i)^{2} \frac{f^{2}}{N^{2}}\right] \\
& +\cdots+\sum_{f=0}^{N-1} \exp \left[(1)^{2} \frac{f^{2}}{N^{2}}\right]+1+\sum_{f=0}^{N-1} \exp \left[(1)^{2} \frac{f^{2}}{N^{2}}\right] \\
& +\cdots+\sum_{f=0}^{N-1} \exp \left[(N-2-i)^{2} \frac{f^{2}}{N^{2}}\right]+\sum_{f=0}^{N-1} \exp \left[(N-1-i)^{2} \frac{f^{2}}{N^{2}}\right]
\end{aligned}
$$

and

$$
\begin{aligned}
p_{i+1}^{2}= & \sum_{f=0}^{N-1} \exp \left[(i+1)^{2} \frac{f^{2}}{N^{2}}\right]+\sum_{f=0}^{N-1} \exp \left[i^{2} \frac{f^{2}}{N^{2}}\right]+\sum_{f=0}^{N-1} \exp \left[(1-i)^{2} \frac{f^{2}}{N^{2}}\right] \\
& +\cdots+\sum_{f=0}^{N-1} \exp \left[(1)^{2} \frac{f^{2}}{N^{2}}\right]+1+\sum_{f=0}^{N-1} \exp \left[(1)^{2} \frac{f^{2}}{N^{2}}\right] \\
& +\cdots+\sum_{f=0}^{N-1} \exp \left[(N-3-i)^{2} \frac{f^{2}}{N^{2}}\right]+\sum_{f=0}^{N-1} \exp \left[(N-2-i)^{2} \frac{f^{2}}{N^{2}}\right] .
\end{aligned}
$$

Hence, $p_{i+1}^{2}$ and $p_{i}^{2}$ have all terms common except the first and last terms. Then

$$
p_{i+1}^{2}-p_{i}^{2}=\sum_{f=0}^{N-1} \exp \left[(i+1)^{2} \frac{f^{2}}{N^{2}}\right]-\sum_{f=0}^{N-1} \exp \left[(N-1-i)^{2} \frac{f^{2}}{N^{2}}\right]
$$

Using Lemma 2,

$$
p_{i+1}^{2}-p_{i}^{2}=\frac{N \sqrt{\pi}}{2}\left(\frac{1}{i+1}-\frac{1}{N-1-i}\right) .
$$

We ignored $\operatorname{Erf}(a)$ since it approaches 1 very quickly (for $a>2$ ) and will not affect the result.

Choosing any value of $i<\frac{N}{2}$ in (6.56) leads to $p_{i+1}^{2}-p_{i}^{2}>0$ and any value of $i>\frac{N}{2}$ leads to $p_{i+1}^{2}-p_{i}^{2}<0$. That verifies our claim in (6.52) and also shows the symmetry of norms $p_{i}$ around $\frac{N}{2}$.

From (6.52) and (6.47), then 


$$
\begin{array}{rll}
i=1 & \text { and } & j=2 \\
& \text { or } & \\
i=N-1 & \text { and } & j=N
\end{array}
$$

solves (6.46). Without loss of generality, we choose $i=1$ and $j=2$. Consequently, (6.45) becomes

$$
\lambda_{\max }\left(A_{G}^{H} A_{G}\right)=\frac{1}{p_{1}^{2} p_{2}^{2}} .
$$

We can express $p_{1}^{2}$ using (6.51) as

$$
\begin{aligned}
p_{1}^{2} & =\sum_{f=0}^{N-1} \exp \left[-\left(\frac{f}{N}\right)^{2}\right]+N+\sum_{a=1}^{M} \sum_{f=0}^{N-1} \exp \left[-a^{2}\left(\frac{f}{N}\right)^{2}\right] \\
& =\frac{N \sqrt{\pi}}{2} \operatorname{Erf}(1)+N+\frac{N \sqrt{\pi}}{2} \sum_{a=1}^{M} \frac{1}{a}
\end{aligned}
$$

where $a=(\tau-i)$. We ignored $\operatorname{Erf}(a)$ since it approaches 1 very fast (for $a>2$ ). The summation in (6.59) reduces to the harmonic sum

$$
\sum_{a=1}^{M} \frac{1}{a}=\log (M)+\gamma+\varepsilon_{M}
$$

where $\gamma=0.5772156649$ is the Euler-Mascheroni constant [70] and $\varepsilon_{M} \sim \frac{1}{2 M}$ which approaches 0 as $M$ goes to infinity. Then, (6.59) becomes

$$
\begin{aligned}
p_{1}^{2} & =\frac{N \sqrt{\pi}}{2} \operatorname{Erf}(1)+N+\frac{N \sqrt{\pi}}{2}(\log (M)+\gamma) \\
& =\frac{N \sqrt{\pi}}{2}\left(\log (M)+c_{1}\right) .
\end{aligned}
$$

where $c_{1}=\operatorname{Erf}(1)+\frac{2}{\sqrt{\pi}}+\gamma=2.5483$.

Similarly,

$$
P_{2}^{2}=\frac{N \sqrt{\pi}}{2}\left(\log (M)+c_{2}\right)
$$

where $c_{2}=\frac{\operatorname{Erf}(2)}{2}+\operatorname{Erf}(1)+\frac{2}{\sqrt{\pi}}+\gamma=3.0460$. 
Hence,

$$
p_{1}^{2} P_{2}^{2}=N^{2} \frac{\pi}{4}\left(\log ^{2}(M)+\left(c_{1}+c_{2}\right) \log (M)+\left(c_{1}+c_{2}\right)\right) .
$$

For large $N$ and reasonably large $M,(6.63)$ can be approximated to

$$
p_{1}^{2} P_{2}^{2}=C^{2} N^{2} \log ^{2}(M)
$$

Hence, (6.58) becomes

$$
\lambda_{\max }\left(A_{G}^{H} A_{G}\right)=\frac{1}{C^{2} N^{2} \log ^{2}(M)} .
$$

Substituting from (6.65) and (6.39), the operator norm of matrix A is expressed as

$$
\left\|A_{G}\right\|=\frac{1}{C N \log (M)} .
$$

Substituting from (6.66) in Lemma 1,

$$
\begin{aligned}
& \mathbb{P}\left(\left|\mathbb{1}^{T} R_{J} A_{G} R_{J} \mathbb{1}-\mathbb{E}^{T} R_{J} A_{G} R_{J} \mathbb{1}\right| \geq t\right) \\
& \quad \leq 2 \exp \left[-c \min \left(\frac{t^{2} N^{2} \log ^{2}(M)}{M}, t N \log (M)\right)\right] .
\end{aligned}
$$

Since $t<1$

$$
\mathbb{P}\left(\left|\mathbb{1}^{T} R_{J} A_{G} R_{J} \mathbb{1}-\mathbb{E}^{T} R_{J} A_{G} R_{J} \mathbb{1}\right| \geq t\right) \leq 2 \exp \left[-c \frac{t^{2} N^{2} \log ^{2} M}{M}\right]
$$

Letting $t=\sqrt{\frac{\log N}{M \log M}}$, we get

$$
\begin{aligned}
& \mathbb{P}\left(\left|\mathbb{1}^{T} R_{J} A_{G} R_{J} \mathbb{1}-\mathbb{E}^{T} R_{J} A_{G} R_{J} \mathbb{1}\right| \geq \sqrt{\frac{\log N}{M \log M}}\right) \leq 2 \exp \left[-c \frac{N^{2}}{M^{2}} \log M \log N\right] \\
& \mathbb{P}\left(\left|\mathbb{1}^{T} R_{J} A_{G} R_{J} \mathbb{1}-\mathbb{E}^{T} R_{J} A_{G} R_{J} \mathbb{1}\right| \geq \sqrt{\frac{\log N}{M \log M}}\right) \leq 2 N^{-c \frac{N^{2}}{M^{2}} \log M} \\
& \mathbb{P}\left(\left|\mathbb{1}^{T} R_{J} A_{G} R_{J} \mathbb{1}-\mathbb{E}^{T} R_{J} A_{G} R_{J} \mathbb{1}\right| \leq \sqrt{\frac{\log N}{M \log M}}\right) \geq 1-2 N^{-c \frac{N^{2}}{M^{2}} \log M}
\end{aligned}
$$


One might choose different values of $t$, However, choosing the value of $t$ will affect the sparsity bound and also affects the probability of the signal recovery guarantees.

\section{Part 2. DFT matrix F}

We should use the same procedures to find the worst case coherence for the DFT matrix. The structure of a full DFT matrix is represented as

$$
F=\left[\begin{array}{ccccc}
1 & 1 & 1 & \cdots & 1 \\
1 & w & w^{2} & \cdots & w^{N-1} \\
1 & w^{2} & w^{4} & \cdots & w^{2(N-1)} \\
\vdots & \vdots & \vdots & \ddots & \vdots \\
1 & w^{(N-1)} & w^{2(N-1)} & \cdots & w^{(N-1)(N-1)}
\end{array}\right]
$$

where

$$
w=e^{-j 2 \pi \frac{n}{N}} .
$$

Since we choose $M$ out of $N$ rows (sub-sampling in frequency), we can calculate the $i$ th column norm as

$$
\left\|F_{i}\right\|_{2}=\sqrt{\sum_{f=0}^{M-1}\left(\exp \left[-j 2 \pi \frac{f n}{N}\right]\right)^{2}}=\sqrt{\sum_{f=0}^{M-1} 1}=\sqrt{M} .
$$

To find the worst case coherence in (6.6), we start by finding the inner product of two columns $i$ and $j$. We will use the same idea used in (6.35) and (6.36) to obtain

$$
\begin{aligned}
\left\langle F_{i}, F_{j}\right\rangle & =F_{i}^{H} F_{j} \\
& =\left(\grave{F}_{i} R_{J} \mathbb{1}\right)^{H}\left(\grave{F}_{j} R_{J} \mathbb{1}\right) \\
& =\left(\mathbb{1}^{T} R_{J} \grave{F}_{i}^{H} \grave{F}_{j} R_{J} \mathbb{1}\right) \\
& =\mathbb{1}^{T} R_{J} A_{F} R_{J} \mathbb{1}
\end{aligned}
$$

where $\grave{F}_{i}$ is a diagonal matrix containing elements of column $i$ as diagonal elements, $R_{J}$ is as defined in Lemma 1 and $\mathbb{1}$ is a unity column vector. Hence,

$$
\begin{aligned}
A_{F} & =\grave{F}_{i}^{H} \grave{F}_{j} \\
& =\operatorname{diag}\left[\frac{1}{M} \exp \left[-j 2 \pi \frac{n_{1}\left(f_{i}-f_{j}\right)}{N}\right] \cdots \cdots \frac{1}{M} \exp \left[-j 2 \pi \frac{n_{N}\left(f_{i}-f_{j}\right)}{N}\right]\right] .
\end{aligned}
$$


Then the operator norm of matrix $A_{F}$ is

$$
\left\|A_{F}\right\|=\sqrt{\lambda_{\max }\left(A_{F}^{H} A_{F}\right)}=\frac{1}{M}
$$

Substituting (6.75) in Lemma 1 gives

$$
\begin{aligned}
& \mathbb{P}\left(\left|\mathbb{1}^{T} R_{J} A_{F} R_{J} \mathbb{1}-\mathbb{E}^{T} R_{J} A_{F} R_{J} \mathbb{1}\right| \geq t\right) \\
& \quad \leq 2 \exp \left[-c \min \left(t^{2} M^{2}, t M\right)\right] .
\end{aligned}
$$

Since $t<1$,

$$
\mathbb{P}\left(\left|\mathbb{1}^{T} R_{J} A_{F} R_{J} \mathbb{1}-\mathbb{E}^{T} R_{J} A_{F} R_{J} \mathbb{1}\right| \geq t\right) \leq 2 \exp \left[-c t^{2} M^{2}\right]
$$

and using the same value of $t=\sqrt{\frac{\log N}{M \log M}}$, we get

$$
\begin{aligned}
& \mathbb{P}\left(\left|\mathbb{1}^{T} R_{J} A_{F} R_{J} \mathbb{1}-\mathbb{E}^{T} R_{J} A_{F} R_{J} \mathbb{1}\right| \geq \sqrt{\frac{\log N}{M \log M}}\right) \leq 2 \exp \left[-c \frac{M \log N}{\log M}\right] \\
& \mathbb{P}\left(\left|\mathbb{1}^{T} R_{J} A_{F} R_{J} \mathbb{1}-\mathbb{E}^{T} R_{J} A_{F} R_{J} \mathbb{1}\right| \geq \sqrt{\frac{\log N}{M \log M}}\right) \leq 2 N^{-c \frac{M}{\log M}} \\
& \mathbb{P}\left(\left|\mathbb{1}^{T} R_{J} A_{F} R_{J} \mathbb{1}-\mathbb{E}^{T} R_{J} A_{F} R_{J} \mathbb{1} \mathbb{1}\right| \leq \sqrt{\frac{\log N}{M \log M}}\right) \geq 1-2 N^{-c \frac{M}{\log M}} .
\end{aligned}
$$

At this point, we have bounds for the worst case coherence for matrix $G$ and $F$ individually. In the next step, as we learned from [62], we combine both matrices bounds (6.69) and (6.78) to form the bound for the S-transform matrix worst case coherence $\mu_{S}$, we obtain

$$
\mathbb{P}\left(\left\langle S_{i}, S_{j}\right\rangle \leq \sqrt{\frac{\log N}{M \log M}}\right) \geq 1-2 N^{-c \frac{N^{2}}{M^{2}} \log M}-2 N^{-c \frac{M}{\log M}} .
$$

Hence, the bound for the S-transform matrix worst case coherence $\mu_{S}$ can be expressed as

$$
\mu_{S} \leq \sqrt{\frac{\log N}{M \log M}}
$$


Using the coherence bound of S-transform matrix in (6.80) with Theorem 2, we obtain the required bound for the maximum number of nonzero elements $K$ as

$$
K \leq \frac{1}{2}+\sqrt{\frac{M \log M}{2 \log N}}
$$

with probability at least

$$
1-2 N^{-c \frac{N^{2}}{M^{2}} \log M}-2 N^{-c \frac{M}{\log M}}
$$

which concludes the proof.

\subsection{Numerical Experiments}

In this section, we study the performance of S-transform compressive sensing in the light of the developed bound. The used parameters are as follows. $N=400$ range cells, $M=255$ measurements and $K_{\max }=11$ as computed from (6.16).

For each experiment, $K$ targets are placed at random delays. Then, white Gaussian noise with zero mean and variance $\sigma^{2}$ is added to the received signal to produce the specified SNR. The reconstruction process is carried out using CVX, a package for specifying and solving convex programs [45].

During the experiment, 100 Monte Carlo simulations are executed. Independent noise realization is used for each execution. The estimated (reconstructed) signal is compared to a threshold. whenever the value at specific delays corresponding to the true target delay exceeds the threshold, it is considered detection. $P_{d}$ is calculated such that all $K$ scatterers are detected. Otherwise, if the estimated signal exceeds the threshold at any other delays, it is considered false alarm. Then, $P_{f a}$ is computed as the average of false alarms over the iterations.

The results are depicted using ROC curves for different values of $P_{d}$ and $P_{f a}$ for a fixed value of SNR. Figure 6.1 and 6.2 depict ROC curves at $\mathrm{SNR}=5$ and 10 respectively.

The comparison is carried out similar to [61] where three values of $K$ are tested as follows. half of the maximum number of detectable targets $\left(K_{\max } / 2\right)$, the maximum number $\left(K_{\max }\right)$ and double the max number $\left(2 * K_{\max }\right)$.

The results show that for different values of SNR, $P_{d}$ increases as $K$ decreases and 


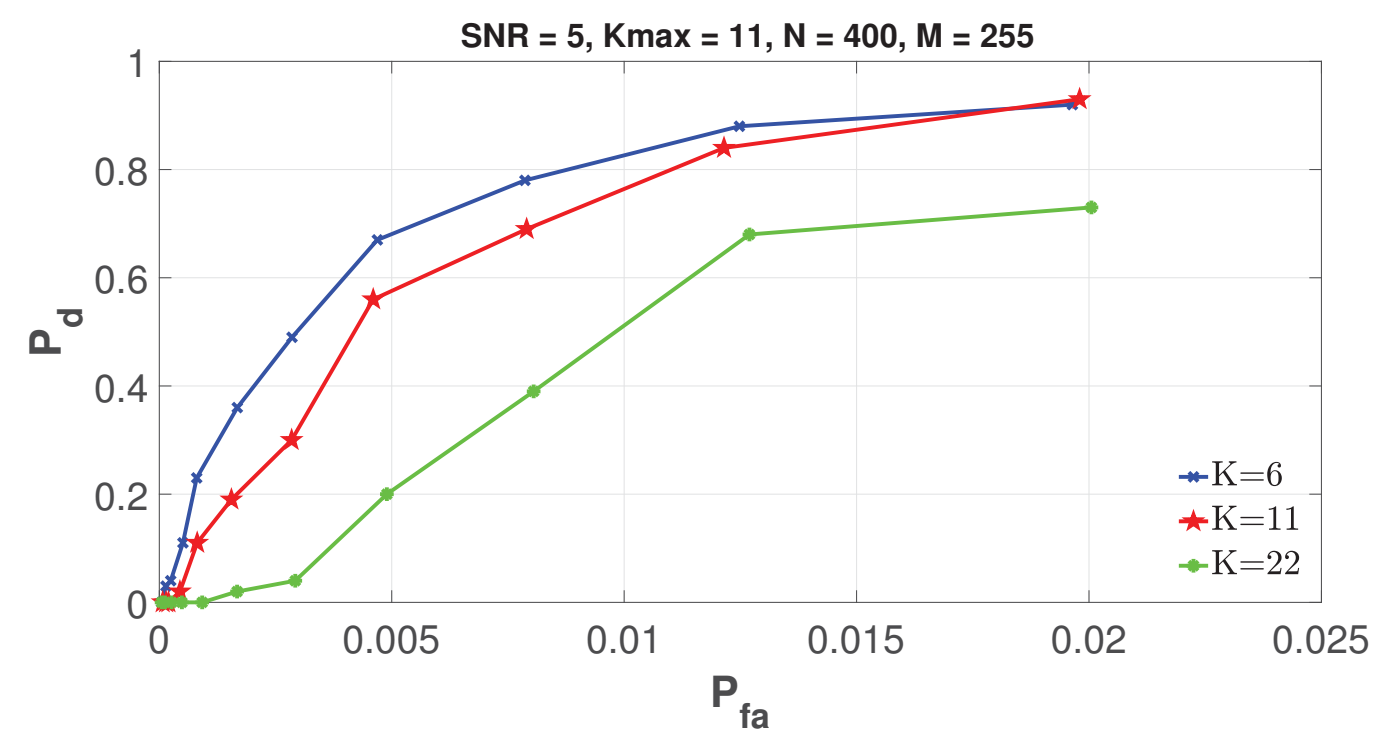

Figure 6.1: Probability of detection vs. probability of false alarm for $\mathrm{SNR}=5 \mathrm{~dB}$, and three values of $K: K \max / 2, K \max , 2 K \max$.

vice versa.

\subsection{Conclusion}

In this chapter, we have tackled the recovery guarantees of compressive sensing from a mathematical point of view. We have derived a mathematical framework for the recovery guarantees of the S-transform matrix scheme. The results yield explicit conditions for the received signal sparsity upper bound which, in our context, is translated to the maximum number of detectable targets $K \leq \frac{1}{2}+\sqrt{\frac{M \log M}{2 \log N}}$. This result shows that the maximum number of detectable targets $K$ is proportional to the number of measurements $M$ and logarithmic with the signal length $N$. However, the main drawback of relying on worst-case coherence only is that the number of measurements $M$ is a quadratic function, not linear, in the number of max detectable targets $K$. Hence, that requires a larger number of measurements to guarantee the uniqueness of the CS recovery algorithm. To find a linear bound, we might consider including the operator norm with the worst case coherence as in Candès and Plan's strategy in Theorem 3 [63] in the future work. 


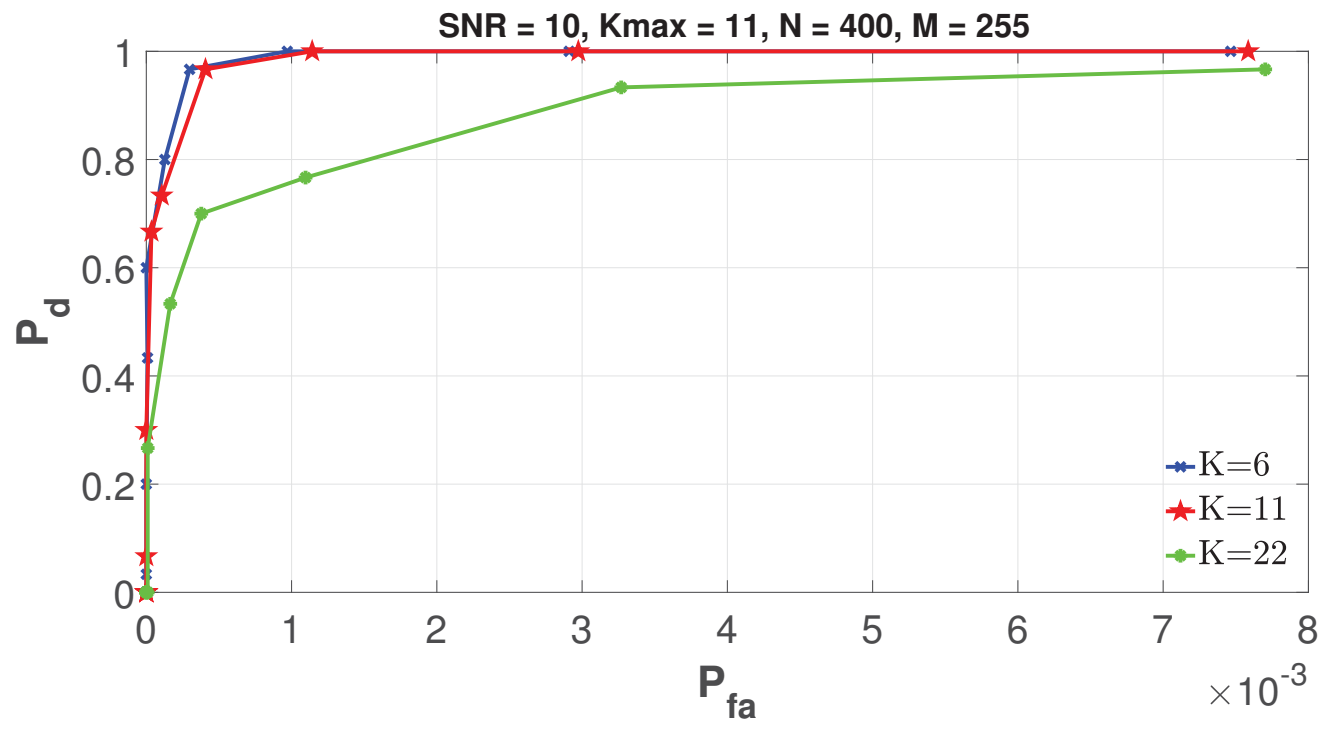

Figure 6.2: Probability of detection vs. probability of false alarm for $\mathrm{SNR}=10 \mathrm{~dB}$, and three values of $K: K \max / 2, K \max , 2 K \max$. 


\section{Chapter 7}

\section{Conclusion and Future Work}

\subsection{Conclusion}

In this thesis, a compressive sensing framework was studied in the context of detecting moving targets in pulse radar signals.

First, we introduced a new formula to improve the utilization of prior information in CS. The proposed formula provided improvements in target probability of detection.

However, the main focus of this thesis was on the reconstruction time of CS algorithms and the ability to extract target range and Doppler quickly. It was noticed that CS recovery algorithms provide an undesired system latency. Hence, we tackled CS using multi-resolution analysis basis functions in order to reduce the reconstruction processing time which causes the system latency.

We started by proposing Haar wavelet bases based algorithm. The results of the proposed algorithm were very promising in the sense of processing speed. The results show a processing time reduction compared to the competing approaches for the same number of measurements. However, the Haar algorithm allowed only for target range detection. It did not allow for target Doppler frequency information extraction. Hence, we proposed using different multi-resolution analysis basis functions that retain the frequency information in order to allow for Doppler frequency extraction. That was by using S-transform basis functions. The results showed almost the same processing time reduction with the ability to detect target range and speed. Moreover, an MTI step was added to the algorithm to provide fixed target cancellation. 
In order to find the maximum number of detectable targets for the proposed Stransform algorithm, we derived a mathematical framework. The proof provided a reasonable upper-bound for the number of detectable targets that guarantees a unique signal recovery with very high probability.

\subsection{Limitations}

In this section, we study the limitations of the proposed algorithms: Haar wavelet algorithm and S-transform algorithm. Since S-transform algorithm provides the capabilities of the Haar wavelet algorithm to extract the targets' range information and also provides Doppler frequency information extraction, we will, mainly, consider the limitations of the S-transform algorithm.

\section{Constant Pulse Repetition Frequency}

In this dissertation, we assume that PRF is constant throughout the observation interval. However, in many radar applications, changing PRF is a preferable option. Frequency agile radars or staggered PRF radars have higher immunity to jamming. Moreover, the target blind speed problem occurs when the target is moving at a relative speed that is proportional to a Doppler frequency equal to a multiple of the PRF. Therefore, changing PRF is an effective solution to overcome the blind speed problem.

Hence, constant PRF limits radar performance. However, we assume constant PRF during the observation time, which controlled by the coherent pulse interval (CPI) (which is equal to a number of PRIs). That means that the proposed algorithm works if we change PRF from CPI to the next one.

\section{Constant Target Delay}

In our work, we assume that the target delay is constant during the observation time. However, that assumption puts some limits on the target speed. The target speed should not exceed a certain limit that makes the echo migrate between range bins during the observation time. We have introduced a detailed explanation of this assumption in Sec. 3.2. We showed that target speed is limited by an upper bound represented as 


$$
\begin{aligned}
v_{t g t} & \leq \frac{c \delta}{2} \frac{1}{\cos \alpha T H} \\
& \text { or } \\
v_{t g t} & \leq \frac{c \delta}{2} \frac{f_{r}}{\cos \alpha H} .
\end{aligned}
$$

where $v_{t g t}$ is the target speed, $c$ is the speed of light, $\delta$ is the pulse width, $f_{r}$ is the PRF, $\alpha$ represents the angle between direction of the reflected signal and the target flight direction and $H$ is represented the number of hits that the target receive during the dwell time, which, in our scenario, is equal to the number of PRIs during the observation time. This bound shows that the observation time affects target speed limit.

Some radar applications, such as military radars, deal with extra fast targets. As an example, the speed of some supersonic military aircraft reach multiples of the speed of sound. In this case, the proposed algorithm will fail to extract the target information.

\section{Constant Target Velocity}

Doppler frequency shift is directly proportional to the target speed. In our work, we consider targets with a constant speed throughout the observation time.

More accurately, we assume constant target relative speed to the radar. Relative speed depends on two parameters. Actual target speed and the angle between the target flight direction and the line of sight between the radar and the target.

Hence, if the target changes its flight direction or accelerates during the observation time, the relative speed will be changed and, consequently, changes the associated Doppler frequency by the relation

$$
f_{d}=\frac{2 v_{r}}{\lambda} \cos \alpha
$$

where $f_{d}$ is the Doppler frequency, $v_{r}$ is the target radial speed, $\lambda$ is the wavelength, and $\alpha$ represents the angle between direction of the reflected signal and the target flight direction.

Therefore, the constant Doppler frequency assumption limits the proposed algorithm performance. The algorithm is still capable of measuring the Doppler frequency 
but it will not be accurate in the case of accelerating targets.

\section{Number of Targets in the Scene}

In this thesis, we assume that the number of targets in the scene is much less than the number of range cells. This assumption allows for considering the received signal as a sparse signal in the time domain. Consequently, we can use compressive sensing platform to analyze it.

However, increasing the number of targets increases the number of nonzero entries in the received signal which decreases the signal sparsity condition. The performance of the compressive sensing recovery algorithms depend, mainly, on the signal sparsity. As we have proved in Chapter. 6 , there is an upper bound for the signal sparsity to guarantee signal reconstruction with high probability.

Hence, increasing the number of targets above that limit degrades the performance of the signal reconstruction and the proposed algorithms performance.

\section{3 $\quad$ Future Work}

In the future work, we consider the following points.

\section{Recovery Guarantees with a Tighter Bound}

In Chapter 6 of this thesis, we have derived a mathematical framework for the recovery guarantees of the S-transform matrix scheme. The results yield explicit conditions for the received signal sparsity upper bound which, in our context, is translated to the maximum number of detectable targets. The results show that the maximum number of detectable targets $K$ is proportional to the number of measurements $M$ and logarithmic with the signal length $N$.

However, the main drawback of the approach that we followed, which relies only on the matrix worst case coherence, is that it produces a bound in which the number of measurements $M$ is a quadratic function, not linear, in the number of max detectable targets $K$. Hence, that requires a larger number of measurements to guarantee the uniqueness of the CS recovery algorithm. Since the main goal of CS is to push the number of measurements to be as close as possible to $K$, we focus in our future work in finding another proof strategy that leads to a tighter bound on the number of 
measurements such as including the operator norm with the worst case coherence as in Candes and Plan strategy in Theorem 3, [63]. As seen from the literature, that theorem provides a bound where the number of measurements is linearly proportional to signal sparsity.

\section{Hardware implementation}

Our future work will be focused on implementing the proposed algorithms. We have studied two approaches for CS hardware implementations, RD and MWC. Since our proposed algorithms are inherited from Xampling framework which is implemented using MWC, our suggestions would be close to the MWC construction. We expect to define two main channels, one for the coarse part and the other for the fine part of the algorithm and choosing the correct basis function would be by using an electronic switch.

\section{Generalization of the proposed algorithms for different radar types}

In this thesis, we have focused on pulse radar signal model. In the future, we are interested in expanding the proposed algorithms to cover different radar types such as multiple input multiple output (MIMO) radar and FMCW radars. The algorithms should also be expanded to deal with 2D signals such as the case with SAR. 


\section{List of References}

[1] Merrill I. Skolnik, Ed., Radar Handbook, 3rd ed. The McGraw-Hill Companies, 2008.

[2] B. R. Mahafza, Radar systems analysis and design using Matlab, 3rd ed. CRC Press, Apr. 2016.

[3] J. N. Laska, S. Kirolos, M. F. Duarte, T. S. Ragheb, R. G. Baraniuk, and Y. Massoud, "Theory and implementation of an analog-to-information converter using random demodulation," in 2007 IEEE International Symposium on Circuits and Systems, May 2007, pp. 1959-1962.

[4] M. Mishali and Y. Eldar, "Xampling: analog data compression," in Data Compression Conference, Mar. 2010, pp. 366-375.

[5] P. G. Casazza and G. Kutyniok, Eds., Finite frames, theory and applications. Springer Science+Business Media New York, 2013.

[6] E. Candes, J. Romberg, and T. Tao, "Robust uncertainty principles: exact signal reconstruction from highly incomplete frequency information," IEEE Trans. Inf. Theory, vol. 52, no. 2, pp. 489-509, Feb. 2006.

[7] I. Immoreev, "Ten questions on UWB [ultra wide band radar]," IEEE Aerospace and Electronic Systems Magazine, vol. 18, no. 11, pp. 8-10, Nov. 2003.

[8] James D. Taylor, Ultrawideband radar: applications and design. CRC Press, May 2012.

[9] R. Baraniuk and P. Steeghs, "Compressive radar imaging," in 2007 IEEE Radar Conference, Apr. 2007, pp. 128-133.

[10] Z. Aly, F. Bonn, and R. Magagi, "Analysis of the backscattering coefficient of salt-affected soils using modeling and RADARSAT-1 SAR data," IEEE Transactions on Geoscience and Remote Sensing, vol. 45, no. 2, pp. 332-341, Feb. 2007.

[11] Y.-S. Yoon and M. Amin, "Through-the-wall radar imaging using compressive sensing along temporal frequency domain," in 2010 IEEE International Conference on Acoustics Speech and Signal Processing (ICASSP), Mar. 2010, pp. 2806-2809. 
[12] M. Leigsnering, C. Debes, and A. Zoubir, "Compressive sensing in throughthe-wall radar imaging," in 2011 IEEE International Conference on Acoustics, Speech and Signal Processing (ICASSP), May 2011, pp. 4008-4011.

[13] A. Gurbuz, J. McClellan, and W. Scott, "GPR imaging using compressed measurements," in Geoscience and Remote Sensing Symposium, 2008. IGARSS 2008. IEEE International, vol. 2, Jul. 2008, pp. II-13-II-16.

[14] A. C. Gurbuz, J. H. McClellan, and W. R. Scott, "A Compressive sensing data acquisition and imaging method for stepped frequency GPRs," IEEE Transactions on Signal Processing, vol. 57, no. 7, pp. 2640-2650, Jul. 2009.

[15] I. Kyriakides, "Adaptive compressive sensing and processing for radar tracking," in 2011 IEEE International Conference on Acoustics, Speech and Signal Processing (ICASSP), May 2011, pp. 3888-3891.

[16] _ - "Adaptive compressive sensing and processing of delay-doppler radar waveforms," IEEE Transactions on Signal Processing, vol. 60, no. 2, pp. 730-739, Feb. 2012.

[17] _ _ "Configurable compressive sensing and processing using tracking information for delay-Doppler radar applications," in 2012 IEEE Radar Conference, May 2012, pp. 0263-0268.

[18] Chen, Victor, Ed., Micro-doppler effect in radar. Artech House Books, Feb. 2011.

[19] D. Donoho, "Compressed sensing," IEEE Trans. Inf. Theory, vol. 52, no. 4, pp. 1289-1306, Apr. 2006.

[20] D. Gabor, "Theory of communication. Part 1: The analysis of information," Journal of the Institution of Electrical Engineers - Part III: Radio and Communication Engineering, vol. 93, no. 26, pp. 429-441, Nov. 1946.

[21] Grchenig, Karlheinz, Foundations of time-frequency analysis, 1st ed. Birkhuser Basel, 2001.

[22] S. Shaobing Chen, D. L. Donoho, and M. A. Saunders, "Atomic decomposition by basis pursuit," Society for Industrial and Applied Mathematics, vol. 43, pp. 129-159, 2001.

[23] I. F. Gorodnitsky and B. D. Rao, "Sparse signal reconstruction from limited data using FOCUSS: a re-weighted minimum norm algorithm," IEEE Transactions on Signal Processing, vol. 45, no. 3, pp. 600-616, Mar. 1997.

[24] S. Mallat and Z. Zhang, "Matching pursuits with time-frequency dictionaries," IEEE Transactions on Signal Processing, vol. 41, no. 12, pp. 3397-3415, Dec. 1993.

[25] J. Tropp and A. Gilbert, "Signal recovery from random measurements via orthogonal matching pursuit," IEEE Transactions on Information Theory, vol. 53, no. 12 , pp. 4655-4666, Dec. 2007. 
[26] J. A. Tropp, "Greed is good: algorithmic results for sparse approximation," IEEE Transactions on Information Theory, vol. 50, no. 10, pp. 2231-2242, Oct. 2004.

[27] — - "Just relax: convex programming methods for identifying sparse signals in noise," IEEE Transactions on Information Theory, vol. 52, no. 3, pp. 1030-1051, Mar. 2006.

[28] D. Needell and J. Tropp, "CoSaMP: Iterative signal recovery from incomplete and inaccurate samples," Appl. Comput. Harmon. Anal., vol. 26, pp. 301-321, May 2009.

[29] Richard G. Baraniuk, "Compressive sensing," IEEE Signal Processing Magazine, pp. 118-124, Jul. 2007.

[30] L. Anitori, M. Otten, and P. Hoogeboom, "Detection performance of compressive sensing applied to radar," in 2011 IEEE Radar Conference (RADAR), May 2011, pp. 200-205.

[31] A. Maleki, L. Anitori, Z. Yang, and R. G. Baraniuk, "Asymptotic analysis of complex LASSO via complex approximate message passing (CAMP)," IEEE Transactions on Information Theory, vol. 59, no. 7, pp. 4290-4308, Jul. 2013.

[32] L. Anitori, M. Otten, W. van Rossum, A. Maleki, and R. Baraniuk, "Compressive CFAR radar detection," in 2012 IEEE Radar Conference (RADAR), May 2012, pp. 0320-0325.

[33] L. Anitori, W. v. Rossum, M. Otten, A. Maleki, and R. Baraniuk, "Compressive sensing radar: Simulation and experiments for target detection," in 21st European Signal Processing Conference (EUSIPCO 2013), Sep. 2013, pp. 1-5.

[34] J. A. Tropp, J. N. Laska, M. F. Duarte, J. K. Romberg, and R. G. Baraniuk, "Beyond Nyquist: efficient sampling of sparse bandlimited signals," IEEE Transactions on Information Theory, vol. 56, no. 1, pp. 520-544, Jan. 2010.

[35] M. Mishali, Y. Eldar, O. Dounaevsky, and E. Shoshan, "Xampling: Analog to digital at sub-Nyquist rates," IET Circuits, Devices Systems, vol. 5, no. 1, pp. 8-20, Jan. 2011.

[36] O. Bar-Ilan and Y. C. Eldar, "Sub-Nyquist radar," in 9th International ITG Conference on Systems, Communication and Coding (SCC), Jan. 2013, pp. 1-6.

[37] N. Wagner, Y. C. Eldar, and Z. Friedman, "Compressed beamforming in ultrasound imaging," IEEE Transactions on Signal Processing, vol. 60, no. 9, pp. 4643-4657, Sep. 2012.

[38] G. Itzhak, E. Baransky, N. Wagner, I. Shmuel, E. Shoshan, and Y. C. Eldar, "A hardware prototype for sub-Nyquist radar sensing," in Proceedings of 2013 9th International ITG Conference on Systems, Communication and Coding (SCC), Jan. 2013, pp. 1-6. 
[39] C. Miosso, R. von Borries, and J. Pierluissi, "Compressive sensing with prior information: requirements and probabilities of reconstruction in 11-minimization," IEEE Transactions on Signal Processing, vol. 61, no. 9, pp. 2150-2164, May 2013.

[40] J. Scarlett, J. Evans, and S. Dey, "Compressed sensing with prior information: information-theoretic limits and practical decoders," IEEE Transactions on Signal Processing, vol. 61, no. 2, pp. 427-439, Jan. 2013.

[41] X. Zhao, T. Lu, and W. Dai, "Compressive sensing reconstruction techniques with magnitude prior information," in Sensor Signal Processing for Defence (SSPD 2011), Sep. 2011, pp. 1-5.

[42] J. Mota, N. Deligiannis, and M. Rodrigues, "Compressed sensing with side information: geometrical interpretation and performance bounds," in 2014 IEEE Global Conference on Signal and Information Processing (GlobalSIP), Dec. 2014, pp. 512-516.

[43] J. Mota, N. Deligiannis, A. Sankaranarayanan, V. Cevher, and M. Rodrigues, "Dynamic sparse state estimation using 11- 11 minimization: Adaptive-rate measurement bounds, algorithms and applications," in 2015 IEEE International Conference on Acoustics, Speech and Signal Processing (ICASSP), Apr. 2015, pp. 3332-3336.

[44] X. Wang, J. Sun, and X. Yang, "Compressive sensing aided the sequential extended Kalman filter tracker for pulse Doppler radar," in 2016 9th International Congress on Image and Signal Processing, BioMedical Engineering and Informatics (CISP-BMEI), Oct. 2016, pp. 1178-1182.

[45] M. Grant and S. Boyd, "CVX: Matlab software for disciplined convex programming," Jun. 2015. [Online]. Available: http://cvxr.com/cvx

[46] S. J. Thiruvengadam, P. Chinnadurai, M. T. T. Kumar, and V. Abhaikumar, "Wavelet based signal processing algorithms for early target detection," in Conference on Convergent Technologies for the Asia-Pacific Region TENCON 2003, vol. 3, Oct. 2003, pp. 1175-1179.

[47] T. Jian, G. Liao, Y. He, and J. Shen, "A CFAR detector based on orthogonal wavelet transform," in 2014 12th International Conference on Signal Processing (ICSP), Oct. 2014, pp. 1963-1967.

[48] D. L. Donoho, "De-noising by soft-thresholding," IEEE Transactions on Information Theory, vol. 41, no. 3, pp. 613-627, May 1995.

[49] G. P. Nason, "A little introduction to wavelets," in 1999/063), IEE Colloquium on Applied Statistical Pattern Recognition (Ref. No, 1999, pp. 1/1-1/6.

[50] R. G. Stockwell, L. Mansinha, and R. P. Lowe, "Localization of the complex spectrum: the S transform," IEEE Transactions on Signal Processing, vol. 44, no. 4, pp. 998-1001, Apr. 1996. 
[51] R.G. Stockwell, "A basis for efficient representation of the S-transform," Digital Signal Processing, vol. 17, no. 1, pp. 371 - 393, 2007.

[52] O. Bar-Ilan and Y. Eldar, "Sub-Nyquist Radar via Doppler Focusing," IEEE Transactions on Signal Processing, vol. 62, no. 7, pp. 1796-1811, Apr. 2014.

[53] A. Moustafa, F. M. Ahmed, K. H. Moustafa, and Y. Halwagy, "A new CFAR processor based on guard cells information," in 2012 IEEE Radar Conference, May 2012, pp. 0133-0137.

[54] A. M. Assem, R. M. Dansereau, and F. M. Ahmed, "Adaptive sub-Nyquist sampling based on haar wavelet and compressive sensing in pulsed radar," in 2016 4th International Workshop on Compressed Sensing Theory and its Applications to Radar, Sonar and Remote Sensing (CoSeRa), Sep. 2016, pp. 173-177.

[55] Simon Foucart, Holger Rauhut, A Mathematical introduction to compressive sensing. Birkhuser, 2013.

[56] T. Blumensath and M. E. Davies, "Iterative hard thresholding for compressed sensing," Applied and Computational Harmonic Analysis, vol. 27, no. 3, pp. 265-274, Nov. 2009. [Online]. Available: http://www.sciencedirect.com/science/ article/pii/S1063520309000384

[57] S. Foucart, "Hard thresholding pursuit: an algorithm for compressive sensing," SIAM Journal on Numerical Analysis, vol. 49, no. 6, pp. 2543-2563, Jan. 2011. [Online]. Available: http://epubs.siam.org/doi/abs/10.1137/100806278

[58] R. Gribonval and M. Nielsen, "Sparse representations in unions of bases," IEEE Transactions on Information Theory, vol. 49, no. 12, pp. 3320-3325, Dec. 2003.

[59] D. L. Donoho, M. Elad, and V. N. Temlyakov, "Stable recovery of sparse overcomplete representations in the presence of noise," IEEE Transactions on Information Theory, vol. 52, no. 1, pp. 6-18, Jan. 2006.

[60] P. Pal and P. P. Vaidyanathan, "Correlation-aware techniques for sparse support recovery," in 2012 IEEE Statistical Signal Processing Workshop (SSP), Aug. 2012 , pp. 53-56.

[61] T. Strohmer and B. Friedlander, "Analysis of sparse MIMO radar," Applied and Computational Harmonic Analysis, vol. 37, no. 3, pp. 361-388, Nov. 2014. [Online]. Available: http://www.sciencedirect.com/science/article/pii/ S1063520314000037

[62] T. Strohmer and H. Wang, "Accurate imaging of moving targets via random sensor arrays and Kerdock codes," Inverse Problems, vol. 29, no. 8, p. 085001 , 2013.

[63] E.J. Cands and Y. Plan, "Near-ideal model selection by 11 minimization," Ann. Statist., vol. 37, pp. 2145-2177, 2009. [Online]. Available: http: //projecteuclid.org/euclid.aos/1247663751 
[64] D. Dorsch and H. Rauhut, "Refined analysis of sparse MIMO radar," Journal of Fourier Analysis and Applications, vol. 23, no. 3, pp. 485-529, Jun. 2017. [Online]. Available: http://link.springer.com/10.1007/s00041-016-9477-7

[65] T. T. Cai and T. Jiang, "Limiting laws of coherence of random matrices with applications to testing covariance structure and construction of compressed sensing matrices," The Annals of Statistics, vol. 39, no. 3, pp. 1496-1525, Jun. 2011. [Online]. Available: http://projecteuclid.org/euclid.aos/1305292044

[66] K. R. Davidson and S. J. Szarek, "Local operator theory, random matrices and banach spaces," Handbook of the Geometry of Banach Spaces, vol. 1, pp. 317-366, Jan. 2001. [Online]. Available: http: //www.sciencedirect.com/science/article/pii/S1874584901800103

[67] W. U. Bajwa, "Geometry of random Toeplitz-block sensing matrices: bounds and implications for sparse signal processing," vol. 8365, 2012, pp. 836 505-836 505-7. [Online]. Available: http://dx.doi.org/10.1117/12.919475

[68] D. L. Hanson and F. T. Wright, "A bound on tail probabilities for quadratic forms in independent random variables," The Annals of Mathematical Statistics, vol. 42, no. 3, pp. 1079-1083, Jun. 1971. [Online]. Available: http://projecteuclid.org/euclid.aoms/1177693335

[69] Rudelson, M., "On the complexity of the set of unconditional convex bodies," Discrete Comput Geom, vol. 55, pp. 185-202, Jan. 2016.

[70] JEFFREY C. LAGARIAS, "Eulers constant: Eulers work and modern developments," Bulletin of the American Mathematical Society, vol. 50, no. 4, pp. 527-628, Oct. 2013. [Online]. Available: http://www.ams.org/journals/ bull/2013-50-04/S0273-0979-2013-01423-X/S0273-0979-2013-01423-X.pdf 